\title{
Intersection theory on moduli spaces of holomorphic bundles of arbitrary rank on a Riemann surface
}

\author{
By Lisa C. Jeffrey and Frances C. Kirwan*
}

\section{Introduction}

Let $n$ and $d$ be coprime positive integers, and define $\mathcal{M}(n, d)$ to be the moduli space of (semi)stable holomorphic vector bundles of rank $n$, degree $d$ and fixed determinant on a compact Riemann surface $\Sigma$. This moduli space is a compact Kähler manifold which has been studied from many different points of view for more than three decades (see for instance Narasimhan and Seshadri [41]). The subject of this article is the characterization of the intersection pairings in the cohomology $\operatorname{ring}^{1} H^{*}(\mathcal{M}(n, d))$. A set of generators of this ring was described by Atiyah and Bott in their seminal 1982 paper [2] on the YangMills equations on Riemann surfaces (where in addition inductive formulas for the Betti numbers of $\mathcal{M}(n, d)$ obtained earlier using number-theoretic methods [13], [25] were rederived). By Poincaré duality, knowledge of the intersection pairings between products of these generators (or equivalently knowledge of the evaluation on the fundamental class of products of the generators) completely determines the structure of the cohomology ring.

In 1991 Donaldson [15] and Thaddeus [47] gave formulas for the intersection pairings between products of these generators in $H^{*}(\mathcal{M}(2,1))$ (in terms of Bernoulli numbers). Then using physical methods, Witten [50] found formulas for generating functions from which could be extracted the intersection pairings between products of these generators in $H^{*}(\mathcal{M}(n, d))$ for general rank $n$. These generalized his (rigorously proved) formulas [49] for the symplectic volume of $\mathcal{M}(n, d)$ : For instance, the symplectic volume of $\mathcal{M}(2,1)$ is given by

$$
\operatorname{vol}(\mathcal{M}(2,1))=\left(1-\frac{1}{2^{2 g-3}}\right) \frac{\zeta(2 g-2)}{2^{g-2} \pi^{2 g-2}}=\frac{2^{g-1}-2^{2-g}}{(2 g-2) !}\left|B_{2 g-2}\right|
$$

*This material is based on work supported by the National Science Foundation under Grant No. DMS-9306029, and by grants from NSERC and FCAR.

${ }^{1}$ Throughout this paper all cohomology groups will have complex coefficients, unless specified otherwise. 
where $g$ is the genus of the Riemann surface, $\zeta$ is the Riemann zeta function and $B_{2 g-2}$ is a Bernoulli number (see [49], [47], [15]). The purpose of this paper is to obtain a mathematically rigorous proof of Witten's formulas for general rank $n$. Our announcement [32] sketched the arguments we shall use, concentrating mainly on the case of rank $n=2$.

The proof involves an application of the nonabelian localization principle [31], [50]. Let $K$ be a compact connected Lie group with Lie algebra $\mathbf{k}$, let $(M, \omega)$ be a compact symplectic manifold equipped with a Hamiltonian action of $K$ and suppose that 0 is a regular value of the moment map $\mu: M \rightarrow \mathbf{k}^{*}$ for this action. One can use equivariant cohomology on $M$ to study the cohomology ring of the reduced space, or symplectic quotient, $M_{\text {red }}=\mu^{-1}(0) / K$, which is an orbifold with an induced symplectic form $\omega_{0}$. In particular, it is shown in [36] that there is a natural surjective homomorphism from the equivariant cohomology $H_{K}^{*}(M)$ of $M$ to the cohomology $H^{*}\left(M_{\text {red }}\right)$ of the reduced space. For any cohomology class $\eta_{0} \in H^{*}\left(M_{\text {red }}\right)$ coming from $\eta \in H_{K}^{*}(M)$ via this map, we derived in [31] a formula (the residue formula, Theorem 8.1 of [31]) for the evaluation $\eta_{0}\left[M_{\text {red }}\right]$ of $\eta_{0}$ on the fundamental class of $M_{\text {red }}$. This formula involves the data that enter the Duistermaat-Heckman formula [17], and its generalization the abelian localization formula [3], [8], [9] for the action of a maximal torus $T$ of $K$ on $M$ : that is, the set $\mathcal{F}$ of connected components $F$ of the fixed point set $M^{T}$ of the action of $T$ on $M$, and the equivariant Euler classes $e_{F}$ of their normal bundles in $M$. Let $\mathbf{t}$ be the Lie algebra of $T$; then the composition $\mu_{T}: M \rightarrow \mathbf{t}^{*}$ of $\mu: M \rightarrow \mathbf{k}^{*}$ with the natural map from $\mathbf{k}^{*}$ to $\mathbf{t}^{*}$ is a moment map for the action of $T$ on $M$. In the case when $K=\mathrm{SU}(2)$ and the order of the stabilizer in $K$ of a generic point of $\mu^{-1}(0)$ is $n_{0}$, the residue formula can be expressed in the form

$$
\eta_{0} e^{\omega_{0}}\left[M_{\text {red }}\right]=\frac{n_{0}}{2} \operatorname{Res}_{X=0}\left((2 X)^{2} \sum_{F \in \mathcal{F}_{+}} h_{F}^{\eta}(X) d X\right)
$$

where the subset $\mathcal{F}_{+}$of $\mathcal{F}$ consists of those components $F$ of the fixed point set $M^{T}$ on which the value taken by the $T$-moment map $\mu_{T}: M \rightarrow \mathbf{t}^{*} \cong \mathbb{R}$ is positive, and for $F \in \mathcal{F}_{+}$the inclusion of $F$ in $M$ is denoted by $i_{F}$ and the meromorphic function $h_{F}^{\eta}$ of $X \in \mathbb{C}$ is defined by

$$
h_{F}^{\eta}(X)=\int_{F} \frac{i_{F}^{*} \eta(X) e^{\bar{\omega}(X)}}{e_{F}(X)}=e^{\mu_{T}(F)(X)} \int_{F} \frac{i_{F}^{*} \eta(X) e^{\omega}}{e_{F}(X)}
$$

when $X \in \mathbb{C}$ has been identified with $\operatorname{diag}(2 \pi i,-2 \pi i) X \in \mathbf{t} \otimes \mathbb{C}$. Here $\bar{\omega}$ is the extension $\omega+\mu$ of the symplectic form $\omega$ on $M$ to an equivariantly closed 2form, while as before $\omega_{0}$ denotes the induced symplectic form on $M_{\text {red }}$. Finally $\operatorname{Res}_{X=0}$ denotes the ordinary residue at $X=0$.

The moduli space $\mathcal{M}(n, d)$ was described by Atiyah and Bott [2] as the symplectic reduction of an infinite-dimensional symplectic affine space $\mathcal{A}$ with 
respect to the action of an infinite-dimensional group $\mathcal{G}$ (the gauge group). ${ }^{2}$ However $\mathcal{M}(n, d)$ can also be exhibited as the symplectic quotient of a finitedimensional symplectic space $M(c)$ by the Hamiltonian action of the finitedimensional group $K=\mathrm{SU}(n)$. One characterization of the space $M(c)$ is that it is the symplectic reduction of the infinite-dimensional affine space $\mathcal{A}$ by the action of the based gauge group $\mathcal{G}_{0}$ (which is the kernel of the evaluation map $\mathcal{G} \rightarrow K$ at a prescribed basepoint: see [28]). Now if a compact group $G$ containing a closed normal subgroup $H$ acts in a Hamiltonian fashion on a symplectic manifold $Y$, then one may "reduce in stages": the space $\mu_{H}^{-1}(0) / H$ has a residual Hamiltonian action of the quotient group $G / H$ with moment $\operatorname{map} \mu_{G / H}: \mu_{H}^{-1}(0) / H \rightarrow(\mathbf{g} / \mathbf{h})^{*}$, and $\mu_{G}^{-1}(0) / G$ is naturally identified as a symplectic manifold with $\mu_{G / H}^{-1}(0) /(G / H)$. Similarly $M(c)$ has a Hamiltonian action of $\mathcal{G} / \mathcal{G}_{0} \cong K$, and the symplectic reduction with respect to this action is identified with the symplectic reduction of $\mathcal{A}$ with respect to the full gauge group $\mathcal{G}$.

Our strategy for obtaining Witten's formulas is to apply nonabelian localization to this extended moduli space $M(c)$, which has a much more concrete (and entirely finite-dimensional) characterization described in Section 4 below. Unfortunately technical difficulties arise, because $M(c)$ is both singular and noncompact. The noncompactness of $M(c)$ causes the more serious problems, the most immediate of which is that there are infinitely many components $F$ of the fixed point set $M(c)^{T}$. These, however, are easy to identify (roughly speaking they correspond to bundles which are direct sums of line bundles), and there are obvious candidates for the equivariant Euler classes of their normal bundles, if the singularities of $M(c)$ are ignored. In the case when $n=2$, for example, a naïve application of the residue formula (1.2), with some sleight of hand, would yield

$$
\begin{aligned}
\operatorname{vol}(\mathcal{M}(2,1)) & =e^{\omega_{0}}[\mathcal{M}(2,1)]=(-1)^{g} \operatorname{Res}_{X=0} \sum_{j=0}^{\infty} \frac{e^{(2 j+1) X}}{2^{g-2} X^{2 g-2}} \\
& =(-1)^{g} \operatorname{Res}_{X=0} \frac{e^{X}}{2^{g-2} X^{2 g-2}\left(1-e^{2 X}\right)} \\
& =(-1)^{g-1} \operatorname{Res}_{X=0} \frac{1}{2^{g-1} X^{2 g-2} \sinh (X)}
\end{aligned}
$$

This does give the correct answer (it agrees with (1.1) above). However it is far from obvious how this calculation might be justified, since the infinite sum does not converge in a neighbourhood of 0 , where the residue is taken, and

\footnotetext{
${ }^{2}$ To obtain his generating functionals, Witten formally applied his version of nonabelian localization to the action of the gauge group on the infinite dimensional space $\mathcal{A}$.
} 
indeed the sum of the residues at 0 of the individual terms in the sum does not converge.

These difficulties can be overcome by making use of a different approach to nonabelian localization given recently by Guillemin-Kalkman [23] and independently by Martin [39]. This is made up of two steps: the first is to reduce to the case of a torus action, and the second, when $K=T$ is a torus, is to study the change in the evaluation on the fundamental class of the reduced space $\mu_{T}^{-1}(\xi) / T$ of the cohomology class induced by $\eta$, as $\xi$ varies in $\mathbf{t}^{*}$. It is in fact an immediate consequence of the residue formula that if $T$ is a maximal torus of $K$ and $\xi \in \mathbf{t}^{*}$ is any regular value sufficiently close to 0 of the $T$-moment map $\mu_{T}: M \rightarrow \mathbf{t}^{*}$, then the evaluation $\eta_{0}\left[M_{\text {red }}\right]$ of $\eta_{0} \in H^{*}\left(M_{\text {red }}\right)$ on the fundamental class of $M_{\text {red }}=\mu^{-1}(0) / K$ is equal to the evaluation of a related element of $H^{*}\left(\mu_{T}^{-1}(\xi) / T\right)$ on the fundamental class of the $T$-reduced space $\mu_{T}^{-1}(\xi) / T$. This was first observed by Guillemin and Kalkman [23] and by Martin [39], who gave an independent proof which showed that $\eta_{0}\left[M_{\text {red }}\right]$ is also equal to an evaluation on

$$
\mu^{-1}(0) / T=\left(M_{\mathbf{t}} \cap \mu_{T}^{-1}(0)\right) / T
$$

where $M_{\mathbf{t}}=\mu^{-1}(\mathbf{t})$. In our situation the space $M_{\mathbf{t}}$ turns out to be "periodic" in a way which enables us to avoid working with infinite sums except in a very trivial sense. This is done by comparing the results of relating evaluations on $\left(M_{\mathbf{t}} \cap \mu_{T}^{-1}(\xi)\right) / T$ for different values of $\xi$ in two ways: using the periodicity and using Guillemin and Kalkman's arguments, which can be made to work in spite of the noncompactness of $M(c)$. The singularities can be dealt with because $M(c)$ is embedded naturally and equivariantly in a nonsingular space, and integrals over $M(c)$ can be rewritten as integrals over this nonsingular space.

In the case when $n=2$ our approach gives expressions for the pairings in $H^{*}(\mathcal{M}(2,1))$ as residues similar to those in (1.3) above. When $n>2$ we consider the action of a suitable one-dimensional subgroup $\hat{T}_{1}$ of $T$, with Lie algebra $\hat{\mathbf{t}}_{1}$ say, on the quotient of $\mu^{-1}\left(\hat{\mathbf{t}}_{1}\right)$ by a subgroup of $T$ whose Lie algebra is a complementary subspace to $\hat{\mathbf{t}}_{1}$ in $\mathbf{t}$. This leads to an inductive formula for the pairings on $H^{*}(\mathcal{M}(n, d))$, and thus to expressions for these pairings as iterated residues (see Theorems 8.1 and 9.12 below, which are the central results of this paper). Witten's formulas, on the other hand, express the pairings as infinite sums over those elements of the weight lattice of $\mathrm{SU}(n)$ which lie in the interior of a fundamental Weyl chamber (see Section 2). These infinite sums are difficult to calculate in general, and there is apparently (see [50, Section 5]) no direct proof even that they are always zero when the pairings they represent vanish on dimensional grounds. However, thanks to an argument of 
Szenes (see Proposition 2.2 below), Witten's formulas can be identified with the iterated residues which appear in our approach.

Over the moduli space $\mathcal{M}(n, d)$ there is a natural line bundle $\mathcal{L}$ (the Quillen line bundle [43]) whose fibre at any point representing a semistable holomorphic bundle $E$ is the determinant line

$$
\operatorname{det} \bar{\partial}=\operatorname{det} H^{1}(\Sigma, E) \otimes \operatorname{det} H^{0}(\Sigma, E)^{*}
$$

of the associated $\bar{\partial}$-operator. Our expressions for pairings in $H^{*}(\mathcal{M}(n, d))$ as iterated residues, together with the Riemann-Roch formula, lead easily (cf. Section 4 of [45]) to a proof of the Verlinde formula for

$$
\operatorname{dim} H^{0}\left(\mathcal{M}(n, d), \mathcal{L}^{k}\right)
$$

for positive integers $k$ (proved by Beauville and Laszlo in [6], by Faltings in [20], by Kumar, Narasimhan and Ramanathan in [38] and by Tsuchiya, Ueno and Yamada in [48]).

This paper is organized as follows. In Section 2 we describe the generators for the cohomology ring $H^{*}(\mathcal{M}(n, d))$ and Witten's formulas for the intersection pairings among products of these generators. In Section 3 we outline tools from the Cartan model of equivariant cohomology, which will be used in later sections, and the different versions of localization which will be relevant. In Section 4 we recall properties of the extended moduli space $M(c)$, and in Section 5 we construct the equivariant differential forms representing equivariant Poincare duals which enable us to rewrite integrals over singular spaces as integrals over ambient nonsingular spaces. Then Section 6 begins the application of nonabelian localization to the extended moduli space, and Section 7 analyses the fixed point sets which arise in this application. Section 8 uses induction to complete the proof of Witten's formulas when the pairings are between cohomology classes of a particular form, Section 9 extends the inductive argument to give formulas for all pairings, and in Section 10 it is shown that these agree with Witten's formulas. Finally as an application, Section 11 gives a proof of the Verlinde formula for $\mathcal{M}(n, d)$.

We would like to thank the Isaac Newton Institute in Cambridge, the Institute for Advanced Study in Princeton, the Institut Henri Poincaré and Université Paris VII, the Green-Hurst Institute for Theoretical Physics in Adelaide and the Massachusetts Institute of Technology for their hospitality during crucial phases in the evolution of this paper. We also thank A. Szenes for pointing out an error in an earlier version of the paper; since the original version of this paper was written, Szenes has obtained new results [46] which are closely related to the results given in Section 11 of our paper. 


\section{The cohomology of the moduli space $\mathcal{M}(n, d)$ and Witten's formulas for intersection pairings}

In order to avoid exceptional cases, we shall assume throughout that the Riemann surface $\Sigma$ has genus $g \geq 2$.

A set of generators for the cohomology $H^{*}(\mathcal{M}(n, d))$ of the moduli space $\mathcal{M}(n, d)$ of stable holomorphic vector bundles of coprime rank $n$ and degree $d$ and fixed determinant on a compact Riemann surface $\Sigma$ of genus $g \geq 2$ is given in [2] by Atiyah and Bott. It may be described as follows. There is a universal rank $n$ vector bundle

$$
\mathbb{U} \rightarrow \Sigma \times \mathcal{M}(n, d)
$$

which is unique up to tensor product with the pullback of any holomorphic line bundle on $\mathcal{M}(n, d)$; for definiteness Atiyah and Bott impose an extra normalizing condition which determines the universal bundle up to isomorphism, but this is not crucial to their argument (see [2, p. 582]). Then by [2, Prop. 2.20] the following elements of $H^{*}(\mathcal{M}(n, d))$ for $2 \leq r \leq n$ make up a set of generators:

$$
\begin{aligned}
f_{r} & =\left([\Sigma], c_{r}(\mathbb{U})\right), \\
b_{r}^{j} & =\left(\alpha_{j}, c_{r}(\mathbb{U})\right), \\
a_{r} & =\left(1, c_{r}(\mathbb{U})\right) .
\end{aligned}
$$

Here, $[\Sigma] \in H_{2}(\Sigma)$ and $\alpha_{j} \in H_{1}(\Sigma)(j=1, \ldots, 2 g)$ form standard bases of $H_{2}(\Sigma, \mathbb{Z})$ and $H_{1}(\Sigma, \mathbb{Z})$, and the bracket represents the slant product $H^{N}(\Sigma \times \mathcal{M}(n, d)) \otimes H_{j}(\Sigma) \rightarrow H^{N-j}(\mathcal{M}(n, d))$. More generally, if $K=\operatorname{SU}(n)$ and $Q$ is an invariant polynomial of degree $s$ on its Lie algebra $\mathbf{k}=s u(n)$ then there is an associated element of $H^{*}(B \mathrm{SU}(n))$ and hence an associated element of $H^{*}(\Sigma \times \mathcal{M}(n, d))$ which is a characteristic class $Q(\mathbb{U})$ of the universal bundle $\mathbb{U}$. Hence the slant product gives rise to classes

$$
\begin{gathered}
([\Sigma], Q(\mathbb{U})) \quad \in \quad H^{2 s-2}(\mathcal{M}(n, d)), \\
\left(\alpha_{j}, Q(\mathbb{U})\right) \in H^{2 s-1}(\mathcal{M}(n, d))
\end{gathered}
$$

and

$$
(1, Q(\mathbb{U})) \in H^{2 s}(\mathcal{M}(n, d)) .
$$

In particular, letting $\tau_{r} \in S^{r}\left(\mathbf{k}^{*}\right)^{K}$ denote the invariant polynomial associated to the $r^{\text {th }}$ Chern class, we recover

$$
\begin{gathered}
f_{r}=\left([\Sigma], \tau_{r}(\mathbb{U})\right), \\
b_{r}^{j}=\left(\alpha_{j}, \tau_{r}(\mathbb{U})\right)
\end{gathered}
$$

\footnotetext{
${ }^{3}$ In this paper, all cohomology groups are assumed to be with complex coefficients.
} 
and

$$
a_{r}=\left(1, \tau_{r}(\mathbb{U})\right)
$$

A special role is played by the invariant polynomial $\tau_{2}=-\langle\cdot, \cdot\rangle / 2$ on $\mathbf{k}$ given by the Killing form or invariant inner product. We normalize the inner product as follows for $K=\mathrm{SU}(n)$ :

$$
\langle X, X\rangle=-\operatorname{Trace}\left(X^{2}\right) /\left(4 \pi^{2}\right) .
$$

The class $f_{2}$ associated to $-\langle\cdot, \cdot\rangle / 2$ is the cohomology class of the symplectic form on $\mathcal{M}(n, d)$.

As was noted in the introduction, Atiyah and Bott identify $\mathcal{M}(n, d)$ with the symplectic reduction of an infinite dimensional affine space $\mathcal{A}$ of connections by the action of an infinite dimensional Lie group $\mathcal{G}$ (the gauge group). They show that associated to this identification there is a natural surjective homomorphism of rings from the equivariant cohomology $\operatorname{ring} H_{\mathcal{G}}^{*}(\mathcal{A})$ to $H^{*}(\mathcal{M}(n, d))$, where $\overline{\mathcal{G}}$ is the quotient of $\mathcal{G}$ by its central subgroup $S^{1}$. There is a canonical $\mathcal{G}$-equivariant universal bundle over $\Sigma \times \mathcal{A}$, and the slant products of its Chern classes with $1 \in H_{0}(\Sigma), \alpha_{j} \in H_{1}(\Sigma)$ for $1 \leq j \leq 2 g$ and [ $\Sigma] \in H_{2}(\Sigma)$ give generators of $H_{\mathcal{G}}^{*}(\mathcal{A})$ which by abuse of notation we shall also call $a_{r}, b_{r}^{j}$ and $f_{r}$. (In fact $H_{\mathcal{G}}^{*}(\mathcal{A})$ is freely generated by $a_{1}, \ldots, a_{n}, f_{2}, \ldots, f_{n}$ and $b_{r}^{j}$ for $1<r \leq n$ and $1 \leq j \leq 2 g$, subject only to the usual commutation relations.) The surjection from $\mathcal{G}$ to $\overline{\mathcal{G}}$ induces an inclusion from $H_{\overline{\mathcal{G}}}^{*}(\mathcal{A})$ to $H_{\mathcal{G}}^{*}(\mathcal{A})$ such that

$$
H_{\mathcal{G}}^{*}(\mathcal{A}) \cong H_{\mathcal{G}}^{*}(\mathcal{A}) \otimes H^{*}\left(B S^{1}\right)
$$

if we identify $H^{*}\left(B S^{1}\right)$ with the polynomial subalgebra of $H_{\mathcal{G}}^{*}(\mathcal{A})$ generated by $a_{1}$, and then the generators $a_{r}, f_{r}$ and $b_{r}^{j}$ for $1<r \leq n$ determine generators of $H_{\overline{\mathcal{G}}}^{*}(\mathcal{A})$ and thus of $H^{*}(\mathcal{M}(n, d))$. These are the generators we shall use in this paper. The normalization condition imposed by Atiyah and Bott corresponds to use of the isomorphism

$$
H_{\mathcal{G}}^{*}(\mathcal{A}) \cong H_{\mathcal{G}^{*}}^{*}(\mathcal{A}) \otimes H^{*}\left(B S^{1}\right)
$$

obtained by identifying $H^{*}\left(B S^{1}\right)$ with the polynomial subalgebra of $H_{\mathcal{G}}^{*}(\mathcal{A})$ generated by $2(g-1) a_{1}+f_{2}$; they choose this condition because it has a nice geometrical interpretation in terms of a universal bundle over $\Sigma \times \mathcal{M}(n, d)$.

In Sections 4 and 5 of [50], Witten obtained formulas for generating functionals from which one may extract all intersection pairings

$$
\prod_{r=2}^{n} a_{r}^{m_{r}} f_{r}^{n_{r}} \prod_{k_{r}=1}^{2 g}\left(b_{r}^{k_{r}}\right)^{p_{r, k_{r}}}[\mathcal{M}(n, d)]
$$

Let us begin with pairings of the form

$$
\prod_{r=2}^{n} a_{r}^{m_{r}} \exp f_{2}[\mathcal{M}(n, d)]
$$


When $m_{r}$ is sufficiently small to ensure convergence of the sum, Witten obtains ${ }^{4}$ (2.4)

$$
\prod_{r=2}^{n} a_{r}^{m_{r}} \exp f_{2}[\mathcal{M}(n, d)]=c^{\rho} \Gamma(-1)^{n_{+}(g-1)}\left(\sum_{\lambda \in \Lambda_{\mathrm{reg}}^{w} \bigcap_{+}} \frac{c^{-\lambda} \prod_{r=2}^{n} \tau_{r}(2 \pi i \lambda)^{m_{r}}}{\mathcal{D}^{2 g-2}(2 \pi i \lambda)}\right),
$$

where

$$
\Gamma=\frac{n^{2 g}}{\# \Pi_{1}\left(K^{\prime}\right)}\left(\frac{\operatorname{vol}\left(K^{\prime}\right)}{(2 \pi)^{\operatorname{dim} K^{\prime}}}\right)^{2 g-2}\left((2 \pi)^{n_{+}} \mathcal{D}(\rho)\right)^{2 g-2}=n^{g}
$$

is a universal constant for $K=\mathrm{SU}(n)$ and $K^{\prime}=K / Z(K)$, and the Weyl odd polynomial $\mathcal{D}$ on $\mathbf{t}^{*}$ is defined by

$$
\mathcal{D}(X)=\prod_{\gamma>0} \gamma(X)
$$

where $\gamma$ runs over the positive roots. Here, $\rho$ is half the sum of the positive roots, and $n_{+}=n(n-1) / 2$ is the number of positive roots. The sum over $\lambda$ in (2.4) runs over those elements of the weight lattice $\Lambda^{w}$ that are in the interior of the fundamental Weyl chamber. ${ }^{5}$ The element

$$
c=e^{2 \pi i d / n} \operatorname{diag}(1, \ldots, 1)
$$

is a generator of the centre $Z(K)$ of $K$; so since $\lambda \in \mathbf{t}^{*}$ is in $\operatorname{Hom}(T, U(1))$, we may evaluate $\lambda$ on $c$ as in (2.4); $c^{\lambda}$ is defined as $\exp \lambda(\tilde{c})$ where $\tilde{c}$ is any element of the Lie algebra of $T$ such that $\exp \tilde{c}=c$. Note that in fact when $d$ is coprime to $n$ (so that when $n$ is even $d$ is odd) we have $c^{\rho}=(-1)^{n-1}$.

Witten's formula [50, (5.21)] covers pairings involving the $f_{r}$ for $r>2$ and the $b_{r}^{j}$ as well as $f_{2}$ and the $a_{r}$. He obtains it by reducing to the special case of pairings of the form (2.3) above (see [50, §5], in particular the calculations (5.11)-(5.20)) and then applying [50, (4.74)] to this special case. In the special case of pairings of the form (2.3), Witten's formula [50, (5.21)] follows from our Theorem 8.1 using Proposition 2.2 below. Moreover our formula (Theorem 9.12) for pairings involving all the generators $a_{r}, b_{r}^{j}$ and $f_{r}$ reduces to the special case just as Witten's does (see Propositions 10.2 and 10.3). Thus Witten's formulas are equivalent to ours, although they look very different (being expressed in terms of infinite sums indexed by dominant weights instead of in terms of iterated residues).

For the sake of concreteness it is worth examining the special case when rank $n=2$ so that the degree $d$ is odd. In fact, since tensoring by a fixed line bundle of degree $e$ induces a homeomorphism between $\mathcal{M}(n, d)$ and

\footnotetext{
${ }^{4}$ In fact $\mathcal{M}(n, d)$ is an $n^{2 g}$-fold cover of the space for which Witten computes pairings; this accounts for the factor $n^{2 g}$ in our formula (2.5). Taking this into account, (2.4) follows from a special case of Witten's formula [50, (5.21)].

${ }^{5}$ The weight lattice $\Lambda^{w} \subset \mathbf{t}^{*}$ is the dual lattice of the integer lattice $\Lambda^{I}=\operatorname{Ker}(\exp )$ in $\mathbf{t}$.
} 
$M(n, d+n e)$, we may assume that $d=1$. In this case the dominant weights $\lambda$ are just the positive integers. The relevant generators of $H^{*}(\mathcal{M}(2,1))$ are

$$
f_{2} \in H^{2}(\mathcal{M}(2,1))
$$

(which is the cohomology class of the symplectic form on $\mathcal{M}(2,1)$ ) and

$$
a_{2} \in H^{4}(\mathcal{M}(2,1))
$$

these arise from the invariant polynomial $\tau_{2}=-\langle\cdot, \cdot\rangle / 2$ by $a_{2}=\tau_{2}(1), f_{2}=$ $\tau_{2}([\Sigma])$ (see (2.1)). We find then that formula (2.4) reduces for $m \leq g-2$ to $^{6}$ $([50,(4.44)])$

$$
a_{2}^{j} \exp \left(f_{2}\right)[\mathcal{M}(2,1)]=\frac{2^{2 g}}{2\left(8 \pi^{2}\right)^{g-1}}\left(\sum_{n=1}^{\infty}(-1)^{n+1} \frac{\pi^{2 j}}{n^{2 g-2-2 j}}\right) .
$$

Thus one obtains the formulas found by Thaddeus in Section 5 of [47] for the intersection pairings $a_{2}^{m} f_{2}^{n}[\mathcal{M}(2,1)]$; these intersection pairings are given by Bernoulli numbers, or equivalently are given in terms of the Riemann zeta function $\zeta(s)=\sum_{n \geq 1} 1 / n^{s}$. As Thaddeus shows in Section 4 of [47], this is enough to determine all the intersection pairings in the case when rank $n$ is two, because all the pairings

$$
a_{2}^{m} f_{2}^{n} \prod_{k=1}^{2 g}\left(b_{2}^{k}\right)^{p_{k}}[\mathcal{M}(2,1)]
$$

are zero except those of the form

$$
a_{2}^{m} f_{2}^{n} b_{2}^{2 i_{1}-1} b_{2}^{2 i_{1}} \ldots b_{2}^{2 i_{q}-1} b_{2}^{2 i_{q}}[\mathcal{M}(2,1)]
$$

where $m+2 n+3 q=3 g-3$ and $1 \leq i_{1}<\cdots<i_{q} \leq g$. This expression equals the evaluation of $a_{2}^{m} f_{2}^{n}$ on the corresponding moduli space of rank 2 and degree 1 bundles over a Riemann surface of genus $g-q$ if $q \leq g-2$, and equals 4 if $q=g-1$.

Szenes [45] has proved that the expression on the right-hand side of (2.4) may be rewritten in a particular form. To state the result we must introduce some notation. The Lie algebra $\mathbf{t}=\mathbf{t}_{n}$ of the maximal torus $T$ of $\mathrm{SU}(n)$ is

$$
\mathbf{t}=\left\{\left(X_{1}, \ldots, X_{n}\right) \in \mathbb{R}^{n}: X_{1}+\cdots+X_{n}=0\right\} .
$$

Define coordinates $Y_{j}=e_{j}(X)=X_{j}-X_{j+1}$ on $\mathbf{t}$ for $j=1, \ldots, n-1$. The positive roots of $\mathrm{SU}(n)$ are then $\gamma_{j k}(X)=X_{j}-X_{k}=Y_{j}+\ldots+Y_{k-1}$ for $1 \leq j<k \leq n$. The integer lattice $\Lambda^{I}$ of $\mathrm{SU}(n)$ is generated by the simple roots $e_{j}, j=1, \ldots, n-1$. The dual lattice to $\Lambda^{I}$ with respect to the inner product $\langle\cdot, \cdot\rangle$ introduced at (2.2) is the weight lattice $\Lambda^{w} \subset \mathbf{t}$; in terms of the inner

${ }^{6}$ Here, we have identified $-a_{2}$ with Witten's class $\Theta$ and $f_{2}$ with Witten's class $\omega$. 
product $\langle\cdot, \cdot\rangle$, it is given by $\Lambda^{w}=\left\{X \in \mathbf{t}: Y_{j} \in \mathbb{Z}\right.$ for $\left.j=1, \ldots, n-1\right\}$. We define also $\Lambda_{\text {reg }}^{w}\left(\mathbf{t}_{n}\right)=\left\{X \in \Lambda^{w}: Y_{j} \neq 0\right.$ for $j=1, \ldots, n-1$ and $\gamma_{j k}(X) \neq 0$ for any $j \neq k\}$.

Definition 2.1. Let $f: \mathbf{t} \otimes \mathbb{C} \rightarrow \mathbb{C}$ be a meromorphic function of the form

$$
f(X)=g(X) e^{-\gamma(X)}
$$

where $\gamma(X)=\gamma_{1} Y_{1}+\cdots+\gamma_{n-1} Y_{n-1}$ for $\left(\gamma_{1}, \ldots, \gamma_{n-1}\right) \in \mathbb{R}^{n-1}$. We define

$$
[[\gamma]]=\left([[\gamma]]_{1}, \ldots,[[\gamma]]_{n-1}\right)
$$

to be the element of $\mathbb{R}^{n-1}$ for which $0 \leq[[\gamma]]_{j}<1$ for all $j=1, \ldots, n-1$ and $[[\gamma]]=\gamma \bmod \mathbb{Z}^{n-1}$. (In other words, $[[\gamma]]=\sum_{j=1}^{n-1}[[\gamma]]_{j} e_{j}$ is the unique element of $\mathbf{t} \cong \mathbb{R}^{n-1}$ which is in the fundamental domain defined by the simple roots for the translation action on $\mathbf{t}_{n}$ of the integer lattice, and which is equivalent to $\gamma$ under translation by the integer lattice.)

We also define the meromorphic function $[[f]]: \mathbf{t} \otimes \mathbb{C} \rightarrow \mathbb{C}$ by

$$
[[f]](X)=g(X) e^{-[[\gamma]](X)}
$$

Proposition 2.2 (Szenes). Let $f: \mathbf{t} \otimes \mathbb{C} \rightarrow \mathbb{C}$ be defined by

$$
f(X)=\frac{\prod_{r=2}^{n} \tau_{r}(X)^{m_{r}} e^{-\tilde{c}(X)}}{\mathcal{D}(X)^{2 g-2}} .
$$

Provided that the $m_{r}$ are sufficiently small to ensure convergence of the sum,

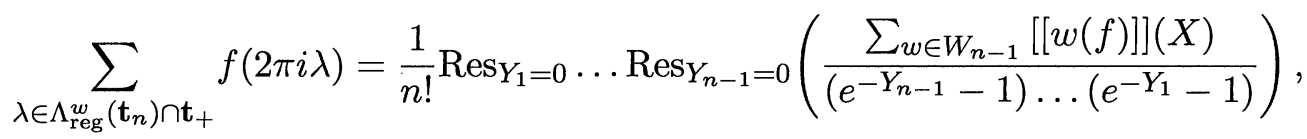
where $W_{n-1} \cong S_{n-1}$ is the Weyl group of $\mathrm{SU}(n-1)$ embedded in $\mathrm{SU}(n)$ in the standard way using the first $n-1$ coordinates $X_{1}, \ldots, X_{n-1}$.

Remark 2.3. Here, we have introduced coordinates $Y_{j}=e_{j}(X)$ on t using the simple roots

$$
\left\{e_{j}: j=1, \ldots n-1\right\}
$$

of $\mathbf{t}$, and $\Lambda_{\text {reg }}^{w}$ denotes the regular part of the weight lattice $\Lambda^{w}$ (see below). Also, we have introduced the unique element $\tilde{c}$ of $\mathbf{t}$ which satisfies $e^{2 \pi i \tilde{c}}=c$ and which belongs to the fundamental domain defined by the simple roots for the translation action on $T_{n}$ of the integer lattice $\Lambda^{I}$. This simply means that $\langle\tilde{c}, X\rangle=\gamma_{1} Y_{1}+\ldots+\gamma_{n-1} Y_{n-1}$ where $0 \leq \gamma_{j}<1$ for $1 \leq j \leq n-1$. (In the notation introduced in Definition 2.1 this says that $\tilde{c}=[[(d / n, d / n, \ldots,-(n-1) d / n)]]$.$) Also, \mathbf{t}_{+}$denotes the fundamental Weyl chamber, which is a fundamental domain for the action of the Weyl group on $\mathbf{t}$. 
If $g\left(Y_{k}, \ldots, Y_{n-1}\right)$ is a meromorphic function of $Y_{k}, \ldots, Y_{n-1}$, we interpret $\operatorname{Res}_{Y_{k}=0} g\left(Y_{k}, \ldots, Y_{n-1}\right)$ as the ordinary one-variable residue of $g$ regarded as a function of $Y_{k}$ with $Y_{k+1}, \ldots, Y_{n-1}$ held constant.

The rest of this section will be devoted to a proof of Proposition 2.2.

We shall prove the following theorem:

TheOREM 2.4. Let $f: \mathbf{t}_{n} \otimes \mathbb{C} \rightarrow \mathbb{C}$ be a meromorphic function of the form $f(X)=g(X) e^{-\gamma(X)}$ where $\gamma(X)=\gamma_{1} Y_{1}+\cdots+\gamma_{n-1} Y_{n-1}$ with $0 \leq \gamma_{n-1}<1$, and $g(X)$ is a rational function of $X$ with poles only on the zeros of the roots $\gamma_{j k}$ and decaying sufficiently fast at infinity. Then

$$
\sum_{\lambda \in \Lambda_{\mathrm{reg}}^{w}\left(\mathbf{t}_{n}\right)} f(2 \pi i \lambda)=\operatorname{Res}_{Y_{1}=0} \ldots \operatorname{Res}_{Y_{n-1}=0}\left(\frac{\sum_{w \in W_{n-1}}[[w(f)]](X)}{\left(e^{-Y_{n-1}}-1\right) \ldots\left(e^{-Y_{1}}-1\right)}\right)
$$

where $W_{n-1}$ is the Weyl group of $\mathrm{SU}(n-1)$ embedded in $\mathrm{SU}(n)$ using the first $n-1$ coordinates.

Remark 2.5. Notice that if $f$ is as in the hypothesis of the theorem (but here one may omit the hypothesis that $0 \leq \gamma_{n-1}<1$ ) then

$$
\sum_{\lambda \in \Lambda_{\mathrm{reg}}^{w}\left(\mathbf{t}_{n}\right)} f(2 \pi i \lambda)=\sum_{\lambda \in \Lambda_{\mathrm{reg}}^{w}\left(\mathbf{t}_{n}\right)}[[f]](2 \pi i \lambda),
$$

where $[[f]]$ is as in Definition 2.1.

Proof of Proposition 2.2 given Theorem 2.4. The function

$$
f(X)=\frac{\prod_{r=2}^{n} \tau_{r}(X)^{m_{r}} e^{-\tilde{c}(X)}}{\mathcal{D}(X)^{2 g-2}}
$$

satisfies the hypotheses of the theorem, provided that the $m_{r}$ are small enough to ensure convergence of the sum. Notice that if $\lambda \in \Lambda_{\text {reg }}^{w}\left(\mathbf{t}_{n}\right)$ then $e^{-2 \pi i \tilde{c}(\lambda)}=$ $c^{-\lambda}$ satisfies $c^{-\lambda}=c^{-w \lambda}$ for all elements $w$ of the Weyl group $W$. Thus for this particular $f$ we have that

$$
\sum_{\lambda \in \Lambda_{\text {reg }}^{w}\left(\mathbf{t}_{n}\right)} f(2 \pi i \lambda)=n ! \sum_{\lambda \in \Lambda_{\text {reg }}^{w}\left(\mathbf{t}_{n}\right) \cap \mathbf{t}_{+}} f(2 \pi i \lambda) .
$$

Thus,

$$
\sum_{\lambda \in \Lambda_{\text {reg }}^{w}\left(\mathbf{t}_{n}\right) \cap \mathbf{t}_{+}} f(2 \pi i \lambda)=\frac{1}{n !} \operatorname{Res}_{Y_{1}=0} \ldots \operatorname{Res}_{Y_{n-1}=0}\left(\frac{\sum_{w \in W_{n-1}}[[w(f)]](X)}{\left(e^{-Y_{n-1}}-1\right) \ldots\left(e^{-Y_{1}}-1\right)}\right)
$$

which is the statement of Proposition 2.2. 
It remains to prove Theorem 2.4. By induction on $n$ it suffices to prove:

LEMma 2.6. Let $f=f_{(n)}: \mathbf{t}_{n} \rightarrow \mathbb{C}$ be as in the statement of Theorem 2.4. Define $f_{(n-1)}: \mathbf{t}_{n-1} \rightarrow \mathbb{C}$ by

$$
f_{(n-1)}\left(Y_{1}, \ldots, Y_{n-2}\right)=\operatorname{Res}_{Y_{n-1}=0} \frac{f\left(Y_{1}, \ldots, Y_{n-1}\right)}{e^{-Y_{n-1}-1}} .
$$

Then

$$
\sum_{\lambda \in \Lambda_{\mathrm{reg}}^{w}\left(\mathbf{t}_{n}\right)} f_{(n)}(2 \pi i \lambda)=\sum_{\lambda \in \Lambda_{\mathrm{reg}}^{w}\left(\mathbf{t}_{n-1}\right)} \sum_{j=1}^{n-1}\left(q_{j} f\right)_{(n-1)}(2 \pi i \lambda),
$$

where $q_{j}$ is the element of the Weyl group $W_{n-1} \cong S_{n-1}$ represented by swapping the coordinates $X_{j}$ and $X_{n-1}$.

Remark 2.7. Note that by Remark 2.5 , the sum $\sum_{j=1}^{n-1}\left(q_{j} f\right)_{(n-1)}(2 \pi i \lambda)$ is equal to

$$
\sum_{j=1}^{n-1}\left[\left[\left(q_{j} f\right)_{(n-1)}\right]\right](2 \pi i \lambda) .
$$

Remark 2.8. Note that the function $\left[\left[\left(q_{j} f\right)_{(n-1)}\right]\right]$ satisfies the hypotheses of Theorem 2.4.

Proof of Lemma 2.6. Let $l_{j}(j=1, \ldots, n-2)$ be integers such that

$$
l_{j}+l_{j+1}+\cdots+l_{k} \neq 0 \text { for any } 1 \leq j \leq k \leq n-2 .
$$

Define $L_{\left(l_{1}, \ldots, l_{n-2}\right)}$ to be the line $\left\{\left(2 \pi i l_{1}, \ldots, 2 \pi i l_{n-2}, Y_{n-1}\right): Y_{n-1} \in \mathbb{C}\right\}$. The condition (2.12) states that all the roots $\gamma_{j k}$ for $1 \leq j<k \leq n-1$ are nonzero on $L_{\left(l_{1}, \ldots, l_{n-2}\right)}$.

Let $f: \mathbf{t} \otimes \mathbb{C} \rightarrow \mathbb{C}$ be a meromorphic function as in the statement of Theorem 2.4, having poles only at the zeros of the roots $\gamma_{j k}$. We shall think of $f$ as a function $f\left(Y_{1}, \ldots, Y_{n-1}\right)$ of the coordinates $Y_{1}, \ldots, Y_{n-1}$. Define $\Lambda_{\left(l_{1}, \ldots, l_{n-2}\right)}^{\mathrm{reg}}$ to be

$$
\begin{gathered}
\Lambda_{\left(l_{1}, \ldots, l_{n-2}\right)}^{\mathrm{reg}}=\left\{X \in L_{\left(l_{1}, \ldots, l_{n-2}\right)}: Y_{j}(X)=X_{j}-X_{j+1} \in 2 \pi i \mathbb{Z},\right. \\
\left.\gamma_{j k}(X) \neq 0 \text { for any } j \neq k\right\} .
\end{gathered}
$$

The sum of all residues of the function $g_{\left(l_{1}, \ldots, \ldots, l_{n-2}\right)}$ on $\mathbb{C}$ given by

$$
g_{\left(l_{1}, \ldots, \ldots, l_{n-2}\right)}\left(Y_{n-1}\right)=\frac{f\left(2 \pi i l_{1}, 2 \pi i l_{2}, \ldots, Y_{n-1}\right)}{e^{-Y_{n-1}}-1}
$$

is zero and these residues occur when $Y_{n-1} \in 2 \pi i \mathbb{Z}$. Therefore we find that the sum

$$
\sum_{p \in \Lambda_{\left(l_{1}, \ldots, l_{n-2}\right)}^{\text {reg }}} f(p)
$$


is given by

$$
\begin{gathered}
\sum_{\left(2 \pi i l_{1}, \ldots, 2 \pi i l_{n-2}, 2 \pi i l_{n-1}\right) \in \Lambda_{\left(l_{1}, \ldots, l_{n-2}\right)}^{\mathrm{reg}}} \operatorname{Res}_{Y_{n-1}=2 \pi i l_{n-1}} \\
\frac{f\left(2 \pi i l_{1}, 2 \pi i l_{2}, \ldots, 2 \pi i l_{n-2}, Y_{n-1}\right)}{e^{-Y_{n-1}-1}} \\
=\sum_{j=1}^{n-2} \operatorname{Res}_{Y_{n-1}=-2 \pi i\left(l_{j}+\ldots+l_{n-2}\right)} \frac{f\left(2 \pi i l_{1}, \ldots, 2 \pi i l_{n-2}, Y_{n-1}\right)}{e^{-Y_{n-1}}-1} \\
+\operatorname{Res}_{Y_{n-1}=0} \frac{f\left(2 \pi i l_{1}, \ldots, 2 \pi i l_{n-2}, Y_{n-1}\right)}{e^{-Y_{n-1}-1}} .
\end{gathered}
$$

Proposition 2.9. Let $p_{j}$ be the point $X \in L_{\left(l_{1}, \ldots, l_{n-2}\right)}$ for which $Y_{n-1}=$ $-2 \pi i\left(l_{j}+\cdots+l_{n-2}\right)$, or equivalently $X_{n}=X_{j}$. Then

$$
\begin{aligned}
\operatorname{Res}_{Y_{n-1}} & =-2 \pi i\left(l_{j}+\ldots+l_{n-2}\right)\left(\frac{f\left(2 \pi i l_{1}, \ldots, 2 \pi i l_{n-2}, Y_{n-1}\right)}{\left.e^{-Y_{n-1}-1}\right)}\right) \\
& =\operatorname{Res}_{Y_{n-1}=0}\left(\frac{\left.q_{j}(f)\left(2 \pi i l_{1}^{(j)}, \ldots, 2 \pi i l_{n-2}^{(j)}, Y_{n-1}\right)\right)}{e^{-Y_{n-1}}-1}\right) .
\end{aligned}
$$

Here, we define an involution $q_{j}: \mathbf{t} \rightarrow \mathbf{t}($ for $j=1, \ldots, n-1)$ by

$$
q_{j}\left(X_{1}, \ldots, X_{j}, \ldots, X_{n-1}, X_{n}\right)=\left(X_{1}, \ldots, X_{j-1}, X_{n-1}, X_{j+1}, \ldots, X_{n-2}, X_{j}, X_{n}\right) .
$$

The integers $l_{1}^{(j)}, \ldots, l_{n-1}^{(j)}$ are defined by the equation

$$
\left.q_{j}(X)\right|_{X=\left(2 \pi i l_{1}, \ldots, 2 \pi i l_{n-2}, Y_{n-1}\right)}=\left(2 \pi i l_{1}^{(j)}, \ldots, 2 \pi i l_{n-2}^{(j)}, 2 \pi i l_{n-1}^{(j)}+Y_{n-1}\right) .
$$

Proof. For $j \leq n-2$, the involution $q_{j}$ is given in the coordinates $\left(Y_{1}, \ldots, Y_{n-1}\right)$ by $q_{j}:\left(Y_{1}, \ldots, Y_{n-1}\right) \mapsto\left(Y_{1}^{\prime}, \ldots, Y_{n-1}^{\prime}\right)$ where $Y_{k}^{\prime}=Y_{k}$ for $k \neq j-1, j, n-2, n-1$ and

$$
\begin{aligned}
Y_{j-1}^{\prime} & =Y_{j-1}+\cdots+Y_{n-2}, \\
Y_{j}^{\prime} & =-\sum_{j \leq k \leq n-2} Y_{k}, \\
Y_{n-2}^{\prime} & =-\sum_{j \leq k \leq n-3} Y_{k} \\
Y_{n-1}^{\prime} & =Y_{j}+\cdots+Y_{n-1} .
\end{aligned}
$$

For $j=n-1, q_{j}$ is the identity map. Notice that $Y_{n-1}^{\prime}$ is the only one of the transformed coordinates that involves $Y_{n-1}$. Notice also that $q_{j}$ takes $p_{j}$ to a point where $Y_{n-1}^{\prime}=0$.

We now examine the image of $L_{\left(l_{1}, \ldots, l_{n-2}\right)}$ under $q_{j}$. The integers $l_{1}^{(j)}, \ldots, l_{n-1}^{(j)}$ were defined by the equation $(2.14)$ : in fact $l_{k}^{(j)}=l_{k}$ for $k \neq$ 
$j-1, j, n-2, n-1$ and

$$
\begin{aligned}
l_{j-1}^{(j)} & =l_{j-1}+\cdots+l_{n-2}, \\
l_{j}^{(j)} & =-\sum_{j \leq k \leq n-2} l_{k}, \\
l_{n-2}^{(j)} & =-\sum_{j \leq k \leq n-3} l_{k}, \\
l_{n-1}^{(j)} & =l_{j}+\cdots+l_{n-2} .
\end{aligned}
$$

We have that

$$
\begin{aligned}
\operatorname{Res}_{Y_{n-1}} & =-2 \pi i\left(l_{j}+\ldots+l_{n-2}\right)\left(\frac{f\left(2 \pi i l_{1}, \ldots, 2 \pi i l_{n-2}, Y_{n-1}\right)}{e^{-Y_{n-1}-1}}\right) \\
& =\operatorname{Res}_{Y_{n-1}=-2 \pi i\left(l_{j}+\ldots+l_{n-2}\right)}\left(\frac{f\left(2 \pi i l_{1}, \ldots, 2 \pi i l_{n-2}, Y_{n-1}\right)}{e^{-Y_{n-1}-2 \pi i\left(l_{j}+\ldots+l_{n-2}\right)}-1}\right)
\end{aligned}
$$

(because $e^{2 \pi i l_{k}}=1$ for all $k=j, \ldots, n-2$ )

$$
=\operatorname{Res}_{Y_{n-1}^{(j)}=0}\left(\frac{q_{j}(f)\left(2 \pi i l_{1}^{(j)}, \ldots, 2 \pi i l_{j-1}^{(j)}, 2 \pi i l_{j}^{(j)}, 2 \pi i l_{j+1}^{(j)}, \ldots, 2 \pi i l_{n-2}^{(j)}, Y_{n-1}^{(j)}\right.}{e^{-Y_{n-1}^{(j)}}-1}\right)
$$

by the formulas $(2.15-2.18)$ where we have defined $Y_{n-1}^{(j)}=Y_{n-1}+$ $2 \pi i\left(l_{j}+\ldots l_{n-2}\right)$ so that $d Y_{n-1}^{(j)}=d Y_{n-1}$. This completes the proof.

Corollary 2.10.

$$
\begin{aligned}
\sum_{p \in \Lambda_{\left(l_{1}, \ldots, l_{n-2}\right)}^{\mathrm{reg}}} f(p)= & \sum_{j=1}^{n-2} \operatorname{Res}_{Y_{n-1}=0}\left(\frac{q_{j}(f)\left(2 \pi i l_{1}^{(j)}, \ldots, 2 \pi i l_{n-2}^{(j)}, Y_{n-1}\right)}{e^{-Y_{n-1}-1}}\right) \\
& +\operatorname{Res}_{Y_{n-1}=0} \frac{f\left(2 \pi i l_{1}, \ldots, 2 \pi i l_{n-2}, Y_{n-1}\right)}{e^{-Y_{n-1}-1}}
\end{aligned}
$$

where the integers $l_{1}^{(j)}, \ldots, l_{n-2}^{(j)}$ are as defined in $(2.19-2.21)$.

Proof. This follows by addition of the results of Proposition 2.9 over all $j=1, \ldots, n-1$; on one side this yields the sum on the right-hand side of (2.13) (which according to (2.13) is equal to $\sum_{p \in \Lambda_{\left(l_{1}, \ldots, l_{n-2}\right)}^{\mathrm{reg}}} f(p)$ ), and on the other side yields the sum on the right-hand side of (2.23).

We shall complete the proof of Lemma 2.6 by summing the equality given in Corollary 2.10 over all possible $\left(l_{1}, \ldots, l_{n-2}\right)$ satisfying $(2.12)$; the proof reduces to the following lemma.

LEMMA 2.11. In the notation of Proposition $2.9,\left(l_{1}^{(j)}, \ldots, l_{n-2}^{(j)}\right) \in$ $\Lambda_{\text {reg }}^{w}\left(\mathbf{t}_{n-1}\right)$. Moreover for any $\left(l_{1}^{\prime}, \ldots, l_{n-2}^{\prime}\right) \in \Lambda_{\text {reg }}^{w}\left(\mathbf{t}_{n-1}\right)$ there is exactly one sequence of integers $\left(l_{1}, \ldots, l_{n-2}\right)$ satisfying $(2.12)$ such that

$$
\left(l_{1}^{(j)}, \ldots, l_{n-2}^{(j)}\right)=\left(l_{1}^{\prime}, \ldots, l_{n-2}^{\prime}\right) .
$$


Proof. This follows immediately from the proof of Proposition 2.9 and the fact that the restriction of $q_{j}$ to $\mathbf{t}_{n-1}$ is given by the action of an element of the Weyl group $W_{n-1}$ and hence maps $\Lambda_{\text {reg }}^{w}\left(\mathbf{t}_{n-1}\right)$ to itself bijectively.

This completes the proof of Lemma 2.6 and hence of Theorem 2.4 and Proposition 2.2.

\section{Residue formulas and nonabelian localization}

Let $(M, \omega)$ be a compact symplectic manifold with a Hamiltonian action of a compact connected Lie group $K$ with Lie algebra $\mathbf{k}$. Let $\mu: M \rightarrow \mathbf{k}^{*}$ be a moment map for this action.

The $K$-equivariant cohomology with complex coefficients $H_{K}^{*}(M)$ of $M$ may be identified with the cohomology of the chain complex

$$
\Omega_{K}^{*}(M)=\left(S\left(\mathbf{k}^{*}\right) \otimes \Omega^{*}(M)\right)^{K}
$$

of equivariant differential forms on $M$, equipped with the differential ${ }^{7}$

$$
(D \eta)(X)=d(\eta(X))-\iota_{X \#}(\eta(X))
$$

where $X^{\#}$ is the vector field on $M$ generated by the action of $X$ (see Chapter 7 of [7]). Here $\left(\Omega^{*}(M), d\right)$ is the de Rham complex of differential forms on $M$ (with complex coefficients), and $S\left(\mathbf{k}^{*}\right)$ denotes the algebra of polynomial functions on the Lie algebra $\mathbf{k}$ of $K$. An element $\eta \in \Omega_{K}^{*}(M)$ may be thought of as a $K$-equivariant polynomial function from $\mathbf{k}$ to $\Omega^{*}(M)$, or alternatively as a family of differential forms on $M$ parametrized by $X \in \mathbf{k}$. The standard definition of degree is used on $\Omega^{*}(M)$ and degree two is assigned to elements of $\mathbf{k}^{*}$.

In fact as a vector space, though not in general as a ring, when $M$ is a compact symplectic manifold with a Hamiltonian action of $K$ then $H_{K}^{*}(M)$ is isomorphic to $H^{*}(M) \otimes H_{K}^{*}$ where $H_{K}^{*}=\Omega_{K}^{*}(\mathrm{pt})=S\left(\mathbf{k}^{*}\right)^{K}$ is the equivariant cohomology of a point (see [36, Prop. 5.8]).

The map $\Omega_{K}^{*}(M) \rightarrow \Omega_{K}^{*}(\mathrm{pt})=S\left(\mathbf{k}^{*}\right)^{K}$ given by integration over $M$ passes to $H_{K}^{*}(M)$. Thus for any $D$-closed element $\eta \in \Omega_{K}^{*}(M)$ representing a cohomology class $[\eta]$, there is a corresponding element $\int_{M} \eta \in \Omega_{K}^{*}(\mathrm{pt})$ which depends only on $[\eta]$. The same is true for any $D$-closed element $\eta=\sum_{j} \eta_{j}$ which is a formal series of elements $\eta_{j}$ in $\Omega_{K}^{j}(M)$ without polynomial dependence on $X$ : we shall in particular consider terms of the form

$$
\eta(X) e^{(\bar{\omega}(X))}
$$

\footnotetext{
${ }^{7}$ This definition of the equivariant cohomology differential differs by a factor of $i$ from that used in [50] but is consistent with that used in [31].
} 
where $\eta \in \Omega_{K}^{*}(M)$ and

$$
\bar{\omega}(X)=\omega+\mu(X) \in \Omega_{K}^{2}(M) .
$$

Here $\mu: M \rightarrow \mathbf{k}^{*}$ is identified in the natural way with a linear function on $\mathbf{k}$ with values in $\Omega^{0}(M)$. It follows directly from the definition of a moment $\operatorname{map}^{8}$ that $D \bar{\omega}=0$.

If $X$ lies in $\mathbf{t}$, the Lie algebra of a chosen maximal torus $T$ of $K$, then there is a formula for $\int_{M} \eta(X)$ (the abelian localization formula [2], [7], [8], [9]) which depends only on the fixed point set of $T$ in $M$. It tells us that

$$
\int_{M} \eta(X)=\sum_{F \in \mathcal{F}} \int \frac{i_{F}^{*} \eta(X)}{e_{F}(X)}
$$

where $\mathcal{F}$ indexes the components $F$ of the fixed point set of $T$ in $M$, the inclusion of $F$ in $M$ is denoted by $i_{F}$ and $e_{F} \in H_{T}^{*}(M)$ is the equivariant Euler class of the normal bundle to $F$ in $M$. In particular, applying (3.3) with $\eta$ replaced by the formal equivariant cohomology class $\eta e^{\bar{\omega}}$ we have

$$
h^{\eta}(X) \stackrel{\text { def }}{=} \int_{M} \eta(X) e^{\bar{\omega}(X)}=\sum_{F \in \mathcal{F}} h_{F}^{\eta}(X)
$$

where

$$
h_{F}^{\eta}(X)=e^{\mu(F)(X)} \int_{F} \frac{i_{F}^{*} \eta(X) e^{\omega}}{e_{F}(X)} .
$$

Note that the moment map $\mu$ takes a constant value $\mu(F) \in \mathbf{t}^{*}$ on each $F \in \mathcal{F}$, and that the integral in (3.5) is a rational function of $X$.

We shall assume throughout that 0 is a regular value of the moment map $\mu: M \rightarrow \mathbf{k}^{*}$; equivalently the action of $K$ on $\mu^{-1}(0)$ has only finite isotropy groups. The reduced space

$$
M_{\text {red }}=\mu^{-1}(0) / K
$$

is then a compact symplectic orbifold. The cohomology (with complex coefficients, as always in this paper) $H^{*}\left(M_{\text {red }}\right)$ of this reduced space is naturally isomorphic to the equivariant cohomology $H_{K}^{*}\left(\mu^{-1}(0)\right)$ of $\mu^{-1}(0)$, and by Theorem 5.4 of [36] the inclusion of $\mu^{-1}(0)$ in $M$ induces a surjection on equivariant cohomology

$$
H_{K}^{*}(M) \rightarrow H_{K}^{*}\left(\mu^{-1}(0)\right) .
$$

Composing we obtain a natural surjection

$$
\Phi: H_{K}^{*}(M) \rightarrow H^{*}\left(M_{\text {red }}\right)
$$

which we shall denote by

$$
\eta \mapsto \eta_{0}
$$

\footnotetext{
${ }^{8}$ We follow the convention that $d \mu(X)=\iota_{X^{\sharp}} \omega$; some authors have $d \mu(X)=-^{\iota} X^{\sharp} \omega$.
} 
When there is no danger of confusion we shall use the same symbol for $\eta \in$ $H_{K}^{*}(M)$ and any equivariantly closed differential form in $\Omega_{K}^{*}(M)$ which represents it. Note that $(\bar{\omega})_{0} \in H^{*}\left(M_{\text {red }}\right)$ is represented by the symplectic form $\omega_{0}$ induced on $M_{\text {red }}$ by $\omega$.

Remark. Later we shall be working with not only the reduced space $M_{\text {red }}=\mu^{-1}(0) / K$ with respect to the action of the nonabelian group $K$, but also $\mu^{-1}(0) / T$ and $M_{\text {red }}^{T}(\xi)=\mu_{T}^{-1}(\xi) / T$ for regular values $\xi$ of the $T$-moment map $\mu_{T}$ which is the composition of $\mu$ with restriction from $\mathbf{k}^{*}$ to $\mathbf{t}^{*}$. We shall use the same notation $\eta_{0}$ for the image of $\eta$ under the surjective homomorphism $\Phi$ for whichever of the spaces $\mu^{-1}(0) / K, \mu^{-1}(0) / T$ or $\mu_{T}^{-1}(0) / T$ we are considering, and the notation $\eta_{\xi}$ if we are working with $\mu_{T}^{-1}(\xi) / T$. It should be clear from the context which version of the map $\Phi$ is being used.

The main result (the residue formula, Theorem 8.1) of [31] gives a formula for the evaluation on the fundamental class $\left[M_{\text {red }}\right] \in H_{*}\left(M_{\text {red }}\right)$, or equivalently (if we represent cohomology classes by differential forms) the integral over $M_{\text {red }}$, of the image $\eta_{0} e^{\omega_{0}}$ in $H^{*}\left(M_{\text {red }}\right)$ of any formal equivariant cohomology class on $M$ of the type $\eta e^{\bar{\omega}}$ where $\eta \in H_{K}^{*}(M)$.

Theorem 3.1 (Residue formula, [31, Th. 8.1]). Let $\eta \in H_{K}^{*}(M)$ induce $\eta_{0} \in H^{*}\left(M_{\text {red }}\right)$. Then

$$
\eta_{0} e^{\omega_{0}}\left[M_{\text {red }}\right]=n_{0} C_{K} \operatorname{Res}\left(\mathcal{D}^{2}(X) \sum_{F \in \mathcal{F}} h_{F}^{\eta}(X)[d X]\right),
$$

where the constant ${ }^{9} C_{K}$ is defined by

$$
C_{K}=\frac{(-1)^{s+n_{+}}}{|W| \operatorname{vol}(T)}
$$

and $n_{0}$ is the order of the stabilizer in $K$ of a generic point ${ }^{10}$ of $\mu^{-1}(0)$.

\footnotetext{
${ }^{9}$ This constant differs by a factor of $(-1)^{s}(2 \pi)^{s-l}$ from that of [31, Th. 8.1]. The reason for the factor of $(2 \pi)^{s-l}$ is that in this paper we shall adopt the convention that weights $\beta \in \mathbf{t}^{*}$ send the integer lattice $\Lambda^{I}=\operatorname{Ker}(\exp : \mathbf{t} \rightarrow T)$ to $\mathbb{Z}$ rather than to $2 \pi \mathbb{Z}$, and that the roots of $K$ are the nonzero weights of its complexified adjoint action. In [31] the roots send $\Lambda^{I}$ to $2 \pi \mathbb{Z}$. The reason for the factor of $(-1)^{s}$ is an error in Section 5 of [31]. In the last paragraph of p.307 of [31] the appropriate form to consider is $\prod_{j=1}^{s}\left(\theta^{j} d z_{j}^{\prime}\right)$, and since 1-forms anticommute this is $(-1)^{s} / i^{s}$ times the term in $\exp \left(i d z^{\prime}(\theta)\right)$ which contributes to the integral (5.4) of [31]. The constant also differs by a factor of $i^{s}$ from that of [33, Th. 3.1], because in that paper the convention adopted on the equivariant cohomology differential is that of [50], not that of [31].

${ }^{10}$ Note that in [31] and [32] $n_{0}$ is stated incorrectly to be the order of the subgroup of $K$ which acts trivially on $\mu^{-1}(0)$ (i.e. the kernel of the action of $K$ on $\mu^{-1}(0)$ ); see the correction in Section 3 of [33]. When $K=T$ is abelian, however, the stabilizer in $K$ of a generic point of $\mu^{-1}(0)$ is equal to the kernel of the action of $K$ on $\mu^{-1}(0)$. Moreover since the coadjoint action of $T$ on $\mathbf{t}^{*}$ is trivial, when $K=T$ this subgroup acts trivially on the normal bundle to $\mu^{-1}(0)$ in $M$ and hence is the kernel of the action of $K$ on $M$.
} 
In this formula $|W|$ is the order of the Weyl group $W$ of $K$, and we have introduced $s=\operatorname{dim} K$ and $l=\operatorname{dim} T$, while $n_{+}=(s-l) / 2$ is the number of positive roots. The measure $[d X]$ on $\mathbf{t}$ and volume $\operatorname{vol}(T)$ of $T$ are obtained from the restriction of a fixed invariant inner product on $\mathbf{k}$, which is used to identify $\mathbf{k}^{*}$ with $\mathbf{k}$ throughout. Also, $\mathcal{F}$ denotes the set of components of the fixed point set of $T$, and if $F$ is one of these components then the meromorphic function $h_{F}^{\eta}$ on $\mathbf{t} \otimes \mathbb{C}$ is defined by (3.5). The polynomial $\mathcal{D}: \mathbf{t} \rightarrow \mathbb{R}$ is defined by

$$
\mathcal{D}(X)=\prod_{\gamma>0} \gamma(X)
$$

where $\gamma$ runs over the positive roots of $K$. Note that it would perhaps be more natural to combine $(-1)^{n_{+}}$from the constant $C_{K}$ with $\mathcal{D}^{2}(X)$ and replace them by the product

$$
\prod_{\gamma} \gamma(X)
$$

of all the positive and negative roots of $K$.

The formula (3.6) was called a residue formula in [31] because the quantity Res (whose general definition was given in Section 8 of [31]) can be expressed as a multivariable residue ${ }^{11}$, whose domain is a class of meromorphic differential forms on $\mathbf{t} \otimes \mathbb{C}$. It is a linear map, but in order to apply it to individual terms in the residue formula some choices have to be made which do not affect the residue of the whole sum. Once the choices have been made one finds that many of the terms in the sum contribute zero, and the formula can be rewritten as a sum over a certain subset $\mathcal{F}_{+}$of the set $\mathcal{F}$ of components of the fixed point set $M^{T}$. When the rank of $K$ is one and $\mathbf{t}$ is identified with $\mathbb{R}$, we can take

$$
\mathcal{F}_{+}=\left\{F \in \mathcal{F}: \mu_{T}(F)>0\right\} .
$$

In this paper we shall be particularly interested in the case where $K$ has rank one, for which the results are as follows.

COROLlaRy 3.2 [35], [51], [31]. In the situation of Theorem 3.1, let $K=U(1)$. Then

$$
\eta_{0} e^{\omega_{0}}\left[M_{\text {red }}\right]=-n_{0} \operatorname{Res}_{X=0}\left(\sum_{F \in \mathcal{F}_{+}} h_{F}^{\eta}(X)\right)
$$

where $n_{0}$ is the order of the stabilizer in $K$ of a generic point in $\mu^{-1}(0)$. Here, the meromorphic function $h_{F}^{\eta}$ on $\mathbb{C}$ is as defined by (3.5), and $\operatorname{Res}_{X=0}$ denotes the coefficient of $1 / X$, where $X \in \mathbb{R}$ has been identified with $2 \pi i X \in \mathbf{k}$.

${ }^{11}$ An alternative definition in terms of iterated 1-variable residues is given in Section 3 of [33]. 
Remark. The notation $\operatorname{Res}_{X=0}$ is intended to indicate the variable $X$ with respect to which the residue is calculated, as well as the point 0 at which the residue is taken, so that, for example, $\operatorname{Res}_{-X=0} f(X)=-\operatorname{Res}_{X=0} f(X)$. It would perhaps be more natural to use the notation $\operatorname{Res}_{X=0} f(X) d X$, but we shall have numerous formulas involving iterated residues of this type which would then become too long and unwieldy.

Corollary 3.3 (cf. [31, Cor. 8.2]). In the situation of Theorem 3.1, let $K=\mathrm{SU}(2)$. Then

$$
\eta_{0} e^{\omega_{0}}\left[M_{\mathrm{red}}\right]=\frac{n_{0}}{2} \operatorname{Res}_{X=0}\left((2 X)^{2} \sum_{F \in \mathcal{F}_{+}} h_{F}^{\eta}(X)\right) .
$$

Here, $n_{0}$, $\operatorname{Res}_{X=0}, h_{F}^{\eta}$ and $\mathcal{F}_{+}$are as in Corollary 3.2 , and $X \in \mathbb{R}$ has been identified with $\operatorname{diag}(2 \pi i,-2 \pi i) X \in \mathbf{t}$.

Remark 3.4. Note that if the degree of $\eta$ is equal to the dimension of $M_{\text {red }}$ then

$$
\eta_{0} e^{\omega_{0}}\left[M_{\text {red }}\right]=\eta_{0}\left[M_{\text {red }}\right] .
$$

Alternatively for $K=U(1)$ or $K=\mathrm{SU}(2)$, if we multiply $\omega$ and $\mu$ by a real scalar $\varepsilon>0$ and let $\varepsilon$ tend to 0 we obtain

$$
\eta_{0}\left[M_{\mathrm{red}}\right]=-n_{0} \operatorname{Res}_{X=0}\left(\sum_{F \in \mathcal{F}_{+}} \int_{F} \frac{i_{F}^{*} \eta(X)}{e_{F}(X)}\right)
$$

or

$$
\eta_{0}\left[M_{\mathrm{red}}\right]=\frac{n_{0}}{2} \operatorname{Res}_{X=0}\left((2 X)^{2} \sum_{F \in \mathcal{F}_{+}} \int_{F} \frac{i_{F}^{*} \eta(X)}{e_{F}(X)}\right) .
$$

The results we have stated so far require the symplectic manifold $M$ to be compact, and this condition is not satisfied in the situation in which we would like to apply these results (in order to obtain formulas for the intersection pairings in the cohomology of moduli spaces of bundles over compact Riemann surfaces). Luckily there are other related results due to Guillemin and Kalkman [23], and independently, Martin [39], which as we shall see can be generalized to noncompact symplectic manifolds.

Guillemin and Kalkman and Martin have approached the problem of finding a formula for

$$
\eta_{0}\left[M_{\mathrm{red}}\right]=\int_{M_{\mathrm{red}}} \eta_{0}
$$

in terms of data on $M$ localised near $M^{T}$ in a slightly different way from that described above. As Guillemin and Kalkman observe, it follows immediately 
from the residue formula that if $\xi \in \mathbf{t}^{*}$ is a regular value of the $T$-moment map $\mu_{T}: M \rightarrow \mathbf{t}^{*}$ which is sufficiently close to 0 then

$$
\eta_{0}\left[M_{\text {red }}\right]=\frac{(-1)^{n_{+}} n_{0}\left(\mathcal{D}^{2} \eta\right)_{\xi}}{n_{0}^{T}|W|}\left[\mu_{T}^{-1}(\xi) / T\right]
$$

where $n_{0}$ (respectively $n_{0}^{T}$ ) is the order of the stabilizer in $K$ (respectively $T$ ) of a generic point of $\mu^{-1}(0)$ (respectively $\mu_{T}^{-1}(0)$ ) and $\mu_{T}^{-1}(\xi) / T$ is the reduced space for the action of $T$ on $M$ with respect to the shifted moment map $\mu_{T}-\xi$. Also $\left(\mathcal{D}^{2} \eta\right)_{\xi} \in H^{*}\left(\mu_{T}^{-1}(\xi) / T\right)$ is the image of $\mathcal{D}^{2} \eta$ under the surjection $\Phi: H_{T}^{*}(M) \rightarrow H^{*}\left(M_{\text {red }}\right)$. Here $\eta \in H_{K}^{*}(M)$ and $\mathcal{D} \in S\left(\mathbf{t}^{*}\right)=H_{T}^{*}$ are regarded as elements of $H_{T}^{*}(M)$ via the natural identification of $H_{K}^{*}(M)$ with the Weyl invariant part $\left(H_{T}^{*}(M)\right)^{W}$ of $H_{T}^{*}(M)$ and the natural inclusion $H_{T}^{*} \rightarrow H_{T}^{*}(M)$. Martin gives a direct proof of (3.8) without appealing to the residue formula, which shows also that for any $\xi$ sufficiently close to 0

$$
\eta_{0}\left[M_{\mathrm{red}}\right]=\frac{n_{0}(\mathcal{D} \eta)_{\xi}}{n_{0}^{\prime}|W|}\left[\mu^{-1}(\xi) / T\right]
$$

where $n_{0}^{\prime}$ is the order of the stabilizer in $T$ of a generic point in $\mu^{-1}(0)$, provided that $\mu^{-1}(\xi) / T$ is oriented appropriately.

Remark 3.5. The symplectic form $\omega$ induces an orientation on $M$, and the induced symplectic forms on $M_{\text {red }}=\mu^{-1}(0) / K$ and on $\mu_{T}^{-1}(\xi) / T$ induce orientations on these quotients. We have made a choice of positive Weyl chamber for $K$ in $\mathbf{t}$; this determines a Borel subgroup $B$ (containing $T$ ) of the complexification $G$ of $K$, such that the weights of the adjoint action of $T$ on the quotient $\mathbf{g} / \mathbf{b}$ of the Lie algebra $\mathbf{g}$ of $G$ by the Lie algebra $\mathbf{b}$ of $B$ are the positive roots of $K$. We then get an orientation of the flag manifold $K / T$ by identifying it with the complex space $G / B$. Modulo the action of finite isotropy groups we have a fibration

$$
\mu^{-1}(0) / T \rightarrow \mu^{-1}(0) / K
$$

with fibre $K / T$; thus the symplectic orientation of $\mu^{-1}(0) / K$ and the orientation of $K / T$ determined by the choice of Weyl chamber induce an orientation of $\mu^{-1}(0) / T$. Since 0 is a regular value of $\mu$, if $\xi$ is sufficiently close to 0 there is a homeomorphism from $\mu^{-1}(0) / T$ to $\mu^{-1}(\xi) / T$ induced by a $T$-equivariant isotopy of $M$; so we get an induced orientation of $\mu^{-1}(\xi) / T$ to be used below.

Note that given a positive Weyl chamber we have another choice of orientation on $\mu^{-1}(\xi) / T$ which is compatible with the symplectic orientation on $\mu_{T}^{-1}(\xi) / T$ and the orientation of the normal bundle to $\mu^{-1}(\xi) / T$ in $\mu_{T}^{-1}(\xi) / T$ induced by identifying it in the natural way with the kernel of the restriction map $\mathbf{k}^{*} \rightarrow \mathbf{t}^{*}$, thence via the fixed invariant inner product on $\mathbf{k}$ with $\mathbf{k} / \mathbf{t}$ and thus finally with the complex vector space $\mathbf{g} / \mathbf{b}$ as above. Because we have 
used the inner product to identify $\mathbf{k} / \mathbf{t}$ with its dual here, this orientation differs from the one chosen above by a factor of $(-1)^{n_{+}}$where $n_{+}$is the number of positive roots.

Proposition 3.6 (Reduction to the abelian case, [S. Martin], [39]). If $T$ is a maximal torus of $K$ and $K$ acts effectively on $M$, then for any regular value $\xi$ of $\mu_{T}$ sufficiently close to 0 , we have that

$$
\begin{aligned}
\int_{\mu^{-1}(0) / K}\left(\eta e^{\bar{\omega}}\right)_{0} & =\frac{n_{0}}{n_{0}^{\prime}|W|} \int_{\mu^{-1}(0) / T}\left(\mathcal{D} \eta e^{\bar{\omega}}\right)_{0}=\frac{n_{0}}{n_{0}^{\prime}|W|} \int_{\mu^{-1}(\xi) / T}\left(\mathcal{D} \eta e^{\bar{\omega}}\right)_{\xi} \\
& =\frac{(-1)^{n_{+}} n_{0}}{n_{0}^{T}|W|} \int_{\mu_{T}^{-1}(\xi) / T}\left(\mathcal{D}^{2} \eta e^{\bar{\omega}}\right)_{\xi}
\end{aligned}
$$

where $n_{0}$ is the order of the stabilizer in $K$ of a generic point of $\mu^{-1}(0)$ and $n_{0}^{T}$ (respectively $n_{0}^{\prime}$ ) is the order of the stabilizer in $T$ of a generic point of $\mu_{T}^{-1}(0)$ (respectively $\mu^{-1}(0)$ ).

Remark 3.7. Note that $(-1)^{n_{+}} \mathcal{D}^{2}$ is the product of all the roots of $K$, both positive and negative.

Martin proves this result by considering the diagram

$$
\begin{aligned}
\mu^{-1}(0) / T & \cong \mu^{-1}(\xi) / T \hookrightarrow \mu_{T}^{-1}(\xi) / T \\
M_{\text {red }}= & \mu^{-1}(0) / K
\end{aligned}
$$

where the homeomorphism from $\mu^{-1}(0) / T$ to $\mu^{-1}(\xi) / T$ is induced by a $T$ equivariant isotopy of $M$ (for $\xi$ sufficiently close to 0 ). For simplicity we shall consider the case when $n_{0}=n_{0}^{\prime}=n_{0}^{T}=1$. As before we use a fixed invariant inner product on $\mathbf{k}$ to identify $\mathbf{k}^{*}$ with $\mathbf{k}$, which splits $T$-equivariantly as the direct sum of $\mathbf{t}$ and its orthogonal complement $\mathbf{t}^{\perp}$. The projection of $\mu: M \rightarrow \mathbf{k}^{*} \cong \mathbf{k}$ onto $\mathbf{t}^{\perp}$ then defines a $T$-equivariant section of the bundle $M \times \mathbf{t}^{\perp}$ on $M$, which has equivariant Euler class $(-1)^{n_{+}} \mathcal{D}$ if we orient $\mathbf{t}^{\perp} \cong$ $\mathbf{k} / \mathbf{t}$ by identifying it with the dual of the complex vector space $\mathbf{g} / \mathbf{b}$ as in Remark 3.5. Hence if $\xi$ is a regular value of $\mu_{T}$ then $\mu^{-1}(\xi) / T$ is a zero-section of the induced orbifold bundle $\mu_{T}^{-1}(\xi) \times_{T} \mathbf{t}^{\perp}$ on $\mu_{T}^{-1}(\xi) / T$, whose Euler class is $(-1)^{n+} \mathcal{D}_{\xi}$. Thus under the conventions for orientations described in Remark 3.5 , evaluating the restriction to $\mu^{-1}(\xi) / T$ of an element of $H^{*}\left(\mu_{T}^{-1}(\xi)\right) / T$ on the fundamental class $\left[\mu^{-1}(\xi) / T\right]$ gives the same result as multiplying by $(-1)^{n_{+}} \mathcal{D}_{\xi}$ and evaluating on the fundamental class $\left[\mu_{T}^{-1}(\xi) / T\right]$.

Now Martin observes that since the natural map

$$
\text { П: } \mu^{-1}(0) / T \rightarrow \mu^{-1}(0) / K=M_{\text {red }}
$$

is a fibration with fibre $K / T$, modulo the action of finite isotropy groups which act trivially on cohomology with complex coefficients, and since the Euler 
characteristic of $K / T$ is nonzero (in fact it is the order $|W|$ of the Weyl group of $K)$, the evaluation of a cohomology class $\eta_{0} \in H^{*}\left(M_{\text {red }}\right)$ on $\left[M_{\text {red }}\right]$ is given by the evaluation of an associated cohomology class on $\left[\mu^{-1}(0) / T\right]$. More precisely we have

$$
\eta_{0}\left[M_{\mathrm{red}}\right]=\frac{e(V)}{|W|} \Pi^{*}\left(\eta_{0}\right)\left[\mu^{-1}(0) / T\right]
$$

where $e(V)$ is the Euler class of the vertical subbundle of the tangent bundle to $\mu^{-1}(0) / T$ with respect to the fibration $\Pi$. As this Euler class is induced by $\mathcal{D}$ under the orientation conventions of Remark 3.5, this completes the proof.

Remark 3.8. In this proof we saw that $\mathcal{D}_{\xi}$ is the cohomology class in $H^{*}\left(\mu_{T}^{-1}(\xi) / T\right)$ which is Poincare dual to the homology class represented by $\mu^{-1}(\xi) / T$. Thus $\mathcal{D}_{\xi}$ may be represented by a closed differential form on $\mu_{T}^{-1}(\xi) / T$ with support in an arbitrarily small neighbourhood of $\mu^{-1}(\xi) / T$. If we interpret $\mathcal{D}_{\xi}$ in this way, Martin's proof of Proposition 3.6 is valid even when $M$ is noncompact and has singularities, provided that for $\xi$ near 0 the subset $\mu^{-1}(\xi)$ is compact and does not meet the singularities of $M$.

Note also that $K$ and hence $T$ act with at most finite isotropy groups on a neighbourhood of $\mu^{-1}(0)$ in $\mu_{T}^{-1}(0)$, and so $\mu_{T}^{-1}(0) / T$ has at worst orbifold singularities in a neighbourhood of $\mu^{-1}(0) / T$. This means that in Proposition 3.6 we do not need to perturb the value of the $T$-moment map $\mu_{T}$ from 0 to a nearby regular value $\xi$ if, as above, we represent $\mathcal{D}_{0}$ by a differential form on $\mu_{T}^{-1}(0) / T$ with support in a sufficiently small neighbourhood of $\mu^{-1}(0) / T$.

This result reduces the problem of finding a formula for $\eta_{0}\left[M_{\text {red }}\right]$ in terms of data on $M$ localized near $M^{T}$ to the case when $K=T$ is itself a torus. Guillemin and Kalkman, and independently Martin, then follow essentially the same line. This is to consider the change in

$$
\eta_{\xi}\left[\mu_{T}^{-1}(\xi) / T\right]
$$

for fixed $\eta \in H_{T}^{*}(M)$, as $\xi$ varies through the regular values of $\mu_{T}$. This is sufficient, if $M$ is a compact symplectic manifold, because the image $\mu_{T}(M)$ is bounded; so if $\xi$ is far enough from 0 then $\mu_{T}^{-1}(\xi) / T$ is empty and thus $\eta_{\xi}\left[\mu_{T}^{-1}(\xi) / T\right]=0$.

More precisely, the convexity theorem of Atiyah [1] and Guillemin and Sternberg [24] tells us that the image $\mu_{T}(M)$ is a convex polytope; it is the convex hull in $\mathbf{t}^{*}$ of the set

$$
\left\{\mu_{T}(F): F \in \mathcal{F}\right\}
$$

of the images $\mu_{T}(F)$ (each a single point of $\mathbf{t}^{*}$ ) of the connected components $F$ of the fixed point set $M^{T}$. This convex polytope is divided by codimensionone "walls" into subpolytopes, themselves convex hulls of subsets of 
$\left\{\mu_{T}(F): F \in \mathcal{F}\right\}$, whose interiors consist entirely of regular values of $\mu_{T}$. When $\xi$ varies in the interior of one of these subpolytopes there is no change in $\eta_{\xi}\left[\mu_{T}^{-1}(\xi) / T\right]$, so it suffices to understand what happens as $\xi$ crosses a codimension-one wall.

Any such wall is the image $\mu_{T}\left(M_{1}\right)$ of a connected component $M_{1}$ of the fixed point set of a circle subgroup $T_{1}$ of $T$. The quotient group $T / T_{1}$ acts on $M_{1}$, which is a symplectic submanifold of $M$, and the restriction of the moment map $\mu_{T}$ to $M_{1}$ has an orthogonal decomposition

$$
\left.\mu_{T}\right|_{M_{1}}=\mu_{T / T_{1}} \oplus \mu_{T_{1}}
$$

where $\mu_{T / T_{1}}: M_{1} \rightarrow\left(\mathbf{t} / \mathbf{t}_{1}\right)^{*}$ is a moment map for the action of $T / T_{1}$ on $M_{1}$ and $\mu_{T_{1}}: M_{1} \rightarrow \mathbf{t}_{1}^{*}$ is constant (because $T_{1}$ acts trivially on $M_{1}$ ). If $\xi_{1}$ is a regular value of $\mu_{T / T_{1}}$ then there is a reduced space

$$
\left(M_{1}\right)_{\text {red }}=\mu_{T / T_{1}}^{-1}\left(\xi_{1}\right) /\left(T / T_{1}\right) .
$$

Guillemin and Kalkman show that if $T$ acts effectively on $M$ (or equivalently if $n_{0}^{T}=1$; see Footnote 9) then, for an appropriate choice of $\xi_{1}$, the change in $\eta_{\xi}\left[\mu_{T}^{-1}(\xi) / T\right]$ as $\xi$ crosses the wall $\mu_{T}\left(M_{1}\right)$ can be expressed as

$$
\left(\operatorname{res}_{M_{1}}(\eta)\right)_{\xi_{1}}\left[\left(M_{1}\right)_{\text {red }}\right]
$$

for a certain residue operation (see Footnote 11 below)

$$
\operatorname{res}_{M_{1}}: H_{T}^{*}(M) \rightarrow H_{T / T_{1}}^{*-d_{1}}\left(M_{1}\right)
$$

where $d_{1}=\operatorname{codim} M_{1}-2$. (Of course care is needed here about the direction in which the wall is crossed; this can be resolved by a careful analysis of orientations.) By induction on the dimension of $T$ this gives a method for calculating $\eta_{\xi}\left[\mu_{T}^{-1}(\xi) / T\right]$ in terms of data on $M$ localized near $M^{T}$.

It is easiest to see how this version of localization is related to the residue formula of [31] in the special case when $K=T=U(1)$. In this case

$$
\Omega_{T}^{*}(M) \cong \mathbb{C}[X] \otimes \Omega^{*}(M)^{T}
$$

is the tensor product of a polynomial ring in one variable $X$ (representing a coordinate function on the Lie algebra $\mathbf{t}$ ) with the algebra of $T$-invariant de Rham forms on $M$. The Guillemin-Kalkman residue operation

$$
\operatorname{res}_{M_{1}}: H_{T}^{*}(M) \rightarrow H_{T / T_{1}}^{*-d_{1}}\left(M_{1}\right)
$$

is then given in terms of the ordinary residue on $\mathbb{C}$ by

$$
\operatorname{res}_{M_{1}}(\eta)=\operatorname{Res}_{X=0} \frac{\left.\eta\right|_{M_{1}}(X)}{e_{M_{1}}(X)}
$$

where $\left.\eta\right|_{M_{1}}(X)$ and the equivariant Euler class $e_{M_{1}}(X)$ of the normal bundle to $M_{1}$ in $M$ are regarded as polynomials in $X$ with coefficients in $H^{*}\left(M_{1}\right)$. 
More precisely we formally decompose this normal bundle (using the splitting principle if necessary) as a sum of complex line bundles $\nu_{j}$ on which $T$ acts with nonzero weights $\beta_{j} \in \mathbf{t}^{*} \cong \mathbb{R}$, and because $c_{1}\left(\nu_{j}\right) \in H^{*}\left(M_{1}\right)$ is nilpotent we can express

$$
\frac{\left.\eta\right|_{M_{1}}(X)}{e_{M_{1}}(X)} \doteq \frac{\left.\eta\right|_{M_{1}}(X)}{\prod_{j}\left(c_{1}\left(\nu_{j}\right)+\beta_{j} X\right)}=\frac{\left.\eta\right|_{M_{1}}(X)}{\prod_{j}\left(\beta_{j} X\right)} \prod_{j}\left(1+\frac{c_{1}\left(\nu_{j}\right)}{\beta_{j} X}\right)^{-1}
$$

as a finite Laurent series in $X$ with coefficients in $H^{*}\left(M_{1}\right)$. Then $\operatorname{res}_{M_{1}}(\eta)$ is simply the coefficient of $1 / X$ in this expression. ${ }^{12}$ Since $T_{1}=T$ acts trivially on $M_{1}$, we have $M_{1, \text { red }}=M_{1}$ and $M_{1}$ is a connected component of the fixed point set $M^{T}$, i.e. $M_{1} \in \mathcal{F}$. Therefore

$$
\left(\operatorname{res}_{M_{1}}(\eta)\right)_{\xi_{1}}\left[\left(M_{1}\right)_{\text {red }}\right]=\operatorname{Res}_{X=0} \int_{M_{1}} \frac{\left.\eta\right|_{M_{1}}(X)}{e_{M_{1}}(X)} .
$$

Of course as $K=T=U(1)$ the convex polytope $\mu_{T}(M)$ in $\mathbf{t}^{*} \cong \mathbb{R}$ is a closed interval, divided into subintervals by the points $\left\{\mu_{T}(F): F \in \mathcal{F}\right\}$. Thus the argument of Guillemin and Kalkman just described, amplified by some careful consideration of orientations, tells us that if $\xi>0$ is a regular value of $\mu_{T}$ and $n_{0}^{T}=1$ then the difference

$$
\eta_{\xi}\left[\mu_{T}^{-1}(\xi) / T\right]-\eta_{0}\left[\mu_{T}^{-1}(0) / T\right]
$$

can be expressed as

$$
\sum_{M_{1} \in \mathcal{F}: 0<\mu_{T}\left(M_{1}\right)<\xi} \operatorname{res}_{M_{1}}(\eta)\left[M_{1}\right]=\operatorname{Res}_{X=0} \sum_{F \in \mathcal{F}: 0<\mu_{T}(F)<\xi} \int_{F} \frac{i_{F}^{*} \eta(X)}{e_{F}(X)}
$$

If we take $\xi>\sup \left(\mu_{T}(M)\right)$ then this gives the same result as Corollary 3.2 (cf. Remark 3.4).

Proposition 3.9 (dependence of symplectic quotients on parameters (Guillemin and Kalkman [23], S. Martin [39])). If $K=T=U(1)$ and $n_{0}^{T}$ is

${ }^{12}$ When the dimension $l$ of $T$ is greater than one the Guillemin-Kalkman residue operation

$$
\operatorname{res}_{M_{1}}: H_{T}^{*}(M) \rightarrow H_{T / T_{1}}^{*-d_{1}}\left(M_{1}\right)
$$

is defined in almost exactly the same way, by choosing a coordinate system $X=\left(X_{1}, \ldots, X_{l}\right)$ on $\mathbf{t}$ where $X_{1}$ is a coordinate on $\mathbf{t}_{1}$, and taking the coefficient of $1 / X_{1}$ in $\frac{\left.\eta\right|_{M_{1}}(X)}{e_{M_{1}}(X)}$ expanded formally as a Laurent series in $X_{1}$ with coefficients in $\mathbb{C}\left[X_{2}, \ldots, X_{l}\right] \otimes \Omega^{*}(M)^{T}$. 
the order of the stabilizer in $T$ of a generic point of $\mu_{T}^{-1}(0)$ then ${ }^{13}$

$$
\begin{aligned}
& \int_{\mu_{T}^{-1}\left(\xi_{1}\right) / T}\left(\eta e^{\bar{\omega}}\right)_{\xi_{1}}-\int_{\mu_{T}^{-1}\left(\xi_{0}\right) / T}\left(\eta e^{\bar{\omega}}\right)_{\xi_{0}} \\
& =n_{0}^{T} \sum_{F \in \mathcal{F}:} \sum_{\xi_{0}<\mu_{T}(F)<\xi_{1}} \operatorname{Res}_{X=0} e^{\mu_{T}(F) X} \int_{F} \frac{\eta(X) e^{\omega}}{e_{F}(X)}
\end{aligned}
$$

where $X \in \mathbb{C}$ has been identified with $2 \pi i X \in \mathbf{t} \otimes \mathbb{C}$ and $\xi_{0}<\xi_{1}$ are two regular values of the moment map.

Remark 3.10. As we have already noted these results can be deduced easily from the residue formula of [31] when $M$ is a compact symplectic manifold. However the proof of Proposition 3.9, just like that of Proposition 3.6 (see Remark 3.8), can be adapted to apply in circumstances when $M$ is not compact and the residue formula of [31] is not valid. Indeed, as Guillemin and Kalkman observe, in the case when $K=T=U(1)$ the basis of their argument applies to any compact oriented $U(1)$-manifold $Y$ with boundary such that the action of $T=U(1)$ on the boundary $\partial Y$ is locally free. Let us suppose for simplicity that $T$ acts effectively on $M$ (i.e. that $n_{0}^{T}=1$; see Footnote 9 ) and let $\zeta$ be a $U(1)$-invariant de Rham one-form on $Y-Y^{T}$ with the property that $\iota_{v}(\zeta)=1$, where the vector field $v$ is the infinitesimal generator of the $U(1)$-action. Guillemin and Kalkman showed that, at the level of forms, the $\operatorname{map} \Phi: H_{T}^{*}(Y) \rightarrow H^{*}(\partial Y / T)$ which is the composition of the restriction map from $H_{T}^{*}(Y)$ to $H_{T}^{*}(\partial Y)$ with the inverse of the canonical isomorphism $H_{T}^{*}(\partial Y) \rightarrow H^{*}(\partial Y / T)$ is given by

$$
\Phi(\eta)=\operatorname{Res}_{X=0} \iota_{v}\left(\frac{\zeta \eta}{X-d \zeta}\right)
$$

(see (1.18) of [23], noting that Guillemin and Kalkman have a different convention for the equivariant cohomology differential, which accounts for the minus sign). If tubular neighbourhoods $U_{1}, \ldots, U_{N}$ of the components $F_{1}, \ldots, F_{N}$ of the fixed point set $Y^{T}$ are removed from $Y$, then Stokes' theorem can be applied to the manifold with boundary $Y-\bigcup_{j=1}^{N} U_{j}$ using the formal identity

$$
D\left(\frac{\zeta \eta}{X-d \zeta}\right)=\eta
$$

on $Y-\bigcup_{j=1}^{N} U_{j}$ to give, after using the fact that $\int_{\partial Y} \alpha=\int_{\partial Y / T} \iota_{v}(\alpha)$ and taking

\footnotetext{
${ }^{13}$ The convention of Guillemin and Kalkman for the sign of the moment map differs from ours (see Footnote 8). This accounts for a difference in sign between their formula and ours.
} 
residues at $X=0$, the formula

$$
\int_{\partial Y / T} \Phi(\eta)=\operatorname{Res}_{X=0} \sum_{j=1}^{N} \int_{F_{j}} \frac{\left.\eta\right|_{F_{j}}(X)}{e_{F_{j}}(X)}
$$

where $e_{F_{j}}$ is the equivariant Euler class of the normal bundle to $F_{j}$ in $Y$.

The formula of Proposition 3.9 comes directly from this when the manifold with boundary $Y$ is $\mu_{T}^{-1}\left[\xi_{0}, \xi_{1}\right]$ for a moment map $\mu_{T}: M \rightarrow \mathbf{t}^{*} \cong \mathbb{R}$ with regular values $\xi_{0}<\xi_{1}$, but there is no need for $\mu_{T}$ to be a moment map or for $M$ to have a symplectic structure for the formula to be valid. It is enough for $\mu_{T}: M \rightarrow \mathbb{R}$ to be a smooth $T$-invariant map with regular values $\xi_{0}<\xi_{1}$ such that $T$ acts freely on the intersections of $\mu_{T}^{-1}\left(\xi_{0}\right)$ and $\mu_{T}^{-1}\left(\xi_{1}\right)$ with the support of the equivariant differential form $\eta$. There is also no need to assume that $M$ is compact; it suffices to suppose that $\mu_{T}: M \rightarrow \mathbb{R}$ is a proper map. Indeed, the assumption that $\mu_{T}$ is proper can itself be weakened; the same proof applies provided only that the intersection of $\mu_{T}^{-1}\left[\xi_{0}, \xi_{1}\right]$ with the support of the equivariant differential form $\eta$ is compact.

\section{Extended moduli spaces}

In [28] certain "extended moduli spaces" of flat connections on a compact Riemann surface with one boundary component are studied. They have natural symplectic structures, and can be used to exhibit the moduli spaces $\mathcal{M}(n, d)$ of interest to us as finite-dimensional symplectic quotients or reduced spaces. Our aim is to obtain Witten's formulas for intersection pairings on $H^{*}(\mathcal{M}(n, d))$ by applying nonabelian localization to these extended moduli spaces. They have a gauge-theoretic description (cf. the introduction to this paper), but we shall use a more concrete (and entirely finite-dimensional) characterization given in [28].

The space with which we want to work is defined by

$$
M(c)=\left(\varepsilon_{K} \times e_{c}\right)^{-1}(\triangle) \subset \operatorname{Hom}(\mathbb{F}, K) \times \mathbf{k},
$$

where $\mathbb{F}$ is the free group on $2 g$ generators $\left\{x_{1}, \ldots, x_{2 g}\right\}$; we identify $\mathbb{F}$ with the fundamental group of the surface $\Sigma$ with one point removed, in such a way that $x_{1}, \ldots, x_{2 g}$ correspond to the generators $\alpha_{1}, \ldots, \alpha_{2 g}$ of $H_{1}(\Sigma, \mathbf{Z})$ chosen in Section 2. Then $\varepsilon_{K}: \operatorname{Hom}(\mathbb{F}, K) \rightarrow K$ is the evaluation map on the relator $r=\prod_{j=1}^{g}\left[x_{j}, x_{j+g}\right]$ and

$$
\varepsilon_{K}\left(h_{1}, \ldots, h_{2 g}\right)=\prod_{j=1}^{g}\left[h_{j}, h_{j+g}\right] .
$$

The map $e_{c}: \mathbf{k} \rightarrow K$ is defined by

$$
e_{c}(Y)=c \exp (Y)
$$


where the generator $c$ of the centre of $K$ is as defined in (2.6) above. The diagonal in $K \times K$ is denoted $\triangle$. The space $M(c)$ then has canonical projection maps $\mathrm{pr}_{1}, \mathrm{pr}_{2}$ which make the following diagram commute:

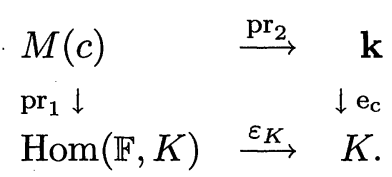

In other words, $M(c)$ is the fibre product of $\operatorname{Hom}(\mathbb{F}, K)$ and $\mathbf{k}$ under the maps $\varepsilon_{K}$ and $e_{c}$. The action of $K$ on $M(c)$ is given by the adjoint actions on $K$ and k. The space $M(c)$ has the following properties (see [28] and [29]):

Proposition 4.1. (a) The space $M(c)$ is smooth near all $(h, \Lambda) \in$ $\operatorname{Hom}(\mathbb{F}, K) \times \mathbf{k}$ for which the linear space $z(h) \cap \operatorname{ker}(d \exp )_{\Lambda} \neq\{0\}$. Here, $z(h)$ is the Lie algebra of the stabilizer $Z(h)$ of $h$.

(b) There is a $K$-invariant 2 -form $\omega$ on $\operatorname{Hom}(\mathbb{F}, K) \times \mathbf{k}$ whose restriction to $M(c)$ is closed and which defines a nondegenerate bilinear form on the Zariski tangent space to $M(c)$ at every $(h, \Lambda)$ in an open dense subset of $M(c)$ containing $M(c) \cap\left(K^{2 g} \times\{0\}\right)$. Thus the form $\omega$ gives rise to a symplectic structure on this open subset of $M(c)$.

(c) With respect to the symplectic structure given by the 2-form $\omega$, a moment map $\mu: M(c) \rightarrow \mathbf{k}^{*}$ for the action of $K$ on $M(c)$ is given by the restriction to $M(c)$ of $-\mathrm{pr}_{2}$, where $\mathrm{pr}_{2}: M(c) \rightarrow \mathbf{k}$ is the projection map to $\mathbf{k}$ (composed with the canonical isomorphism $\mathbf{k} \rightarrow \mathbf{k}^{*}$ given by the invariant inner product on $\mathbf{k})$.

(d) The space $M(c)$ is smooth in a neighbourhood of $\mu^{-1}(0)$.

(e) The symplectic quotient $M_{\mathrm{red}}=M(c) \cap \mu^{-1}(0) / K$ can be naturally identified with $\varepsilon_{K}^{-1}(c) / K=\mathcal{M}(n, d)$.

Remark 4.2. We shall also use $\mu$ to denote the map

$$
\mu: K^{2 g} \times \mathbf{k} \rightarrow \mathbf{k}
$$

defined by

$$
\mu(h, \Lambda)=-\Lambda,
$$

even though it is only its restriction to $M(c)$ which is a moment map in any obvious sense. That is why we write $M(c) \cap \mu^{-1}(0) / K$ instead of $\mu^{-1}(0) / K$ in (e) above.

Remark 4.3. Using our description (4.4) of $M(c)$ as a fibre product, it is easy to identify the components $F$ of the fixed point set of the action of $T$. We examine the fixed point sets of the action of $T$ on $\operatorname{Hom}(\mathbb{F}, K)$ and $\mathbf{k}$ and 
find

$$
\begin{aligned}
& M(c)^{T} \quad \stackrel{\mathrm{pr}_{2}}{\longrightarrow} \quad \mathbf{t} \\
& \mathrm{pr}_{1} \downarrow \text {. } \quad \downarrow \mathrm{e}_{\mathrm{c}} \\
& \operatorname{Hom}(\mathbb{F}, T) \stackrel{\varepsilon_{K}}{\longrightarrow} 1 \in T .
\end{aligned}
$$

(Notice that $\varepsilon_{K}$ sends $\operatorname{Hom}(\mathbb{F}, T)$ to 1 because $T$ is abelian.) Thus

$$
M(c)^{T}=\operatorname{Hom}(\mathbb{F}, T) \times e_{c}^{-1}(1)=T^{2 g} \times\left\{\delta-\tilde{c}: \quad \delta \in \Lambda^{I} \subset \mathbf{t}\right\}
$$

where $\tilde{c}$ is a fixed element of $\mathbf{t}$ for which $\exp \tilde{c}=c$. (Here, $\Lambda^{I}$ denotes the integer lattice $\operatorname{Ker}(\exp ) \subset \mathbf{t}$.) If we ignore the singularities of $M(c)$, this description also enables us to find a plausible candidate for the equivariant Euler class $e_{F_{\delta}}$ of the normal bundle of each component $T^{2 g} \times(\delta-\tilde{c})$ in $M(c)^{T}$ (indexed by $\left.\delta \in \Lambda^{I}\right)$. This should be simply the equivariant Euler class of the normal bundle to $T^{2 g}$ in $K^{2 g}$, implying that $e_{F_{\delta}}$ is in fact independent of $\delta$ and is given by

$$
e_{F_{\delta}}(X)=\left(\prod_{\gamma} \gamma\right)^{g}=\left((-1)^{n_{+}} \mathcal{D}(X)^{2}\right)^{g} .
$$

The symplectic volume of the component $F_{\delta}$ is independent of $\delta$ (indeed these components are all identified symplectically with $T^{2 g}$ ); we denote the volume of $F_{\delta}$ by $\int_{F} e^{\omega}$. The constant value taken by the moment map $\mu_{T}$ on the component $F=F_{\delta}$ is given by $\tilde{c}-\delta$.

We shall need also the following property (proved in [30]):

Proposition 4.4. The generating classes $a_{r}, b_{r}^{j}$ and $f_{r}(r=2, \ldots, n$, $j=1, \ldots, 2 g)$ extend to classes $\tilde{a}_{r}(X), \tilde{b}_{r}^{j}(X)$ and $\tilde{f}_{r}(X) \in H_{K}^{*}(M(c))$.

Indeed, because of our conventions on the equivariant differential, the construction of [30] (which will be described at the beginning of Section 9) tells us that the equivariant differential form $\tilde{a}_{r}(X) \in \Omega_{K}^{*}(M(c))$ whose restriction represents the cohomology class $a_{r} \in H^{*}(\mathcal{M}(n, d))$ is $\tau_{r}(-X)$, where as above $\tau_{r} \in S^{r}\left(\mathbf{k}^{*}\right)^{K} \cong H_{K}^{*}(\mathrm{pt})$ is the invariant polynomial which is associated to the $r^{\text {th }}$ Chern class (see [30]). Moreover $\tilde{f}_{2}$ is the extension $\bar{\omega}=\omega+\mu$ of the symplectic form $\omega$ to an equivariantly closed differential form (see [30] again).

Finally we shall need to work with the symplectic subspace $M_{\mathbf{t}}(c)=$ $M(c) \cap \mu^{-1}(\mathbf{t})$ of $M(c)$, which is no longer acted on by $K$ but is acted on by $T$. The space $M_{\mathbf{t}}(c)$ has an important periodicity property:

Lemma 4.5. Suppose $\Lambda_{0}$ lies in the integer lattice $\Lambda^{I}=\operatorname{Ker}(\exp )$ in $\mathbf{t}$. Then there is a homeomorphism $s_{\Lambda_{0}}: K^{2 g} \times \mathbf{k} \rightarrow K^{2 g} \times \mathbf{k}$ defined by

$$
s_{\Lambda_{0}}:(h, \Lambda) \mapsto\left(h, \Lambda+\Lambda_{0}\right)
$$

which restricts to a homeomorphism $s_{\Lambda_{0}}: M_{\mathbf{t}}(c) \rightarrow M_{\mathbf{t}}(c)$. 
Proof. This is an immediate consequence of the definition of $M_{\mathbf{t}}(c)$ and the fact that $\exp \left(\Lambda+\Lambda_{0}\right)=\exp (\Lambda) \exp \left(\Lambda_{0}\right)$ when $\Lambda$ and $\Lambda_{0}$ commute.

Let us examine the behaviour of the images in $H_{T}^{*}\left(M_{\mathbf{t}}(c)\right)$ of these extensions $\tilde{a}_{r}(X), \tilde{b}_{r}^{j}(X), \tilde{f}_{r}(X) \in H_{K}^{*}(M(c))$ of the generating classes $a_{r}, b_{r}^{j}, f_{r}$ (see Proposition 4.4) under pullback under these homeomorphisms $s_{\Lambda_{0}}: M_{\mathbf{t}}(c) \rightarrow$ $M_{\mathbf{t}}(c)$. By abuse of language, we shall refer to these images also as $\tilde{a}_{r}(X), \tilde{b}_{r}^{j}(X)$ and $\tilde{f}_{r}(X)$. We noted above that the classes $\tilde{a}_{r}(X)$ are the images in $H_{K}^{*}(M(c))$ of the polynomials $\tau_{r}(-X) \in H_{K}^{*}=S\left(\mathbf{k}^{*}\right)^{K}$ (cf. (2.1)). Moreover (by [30, (8.18)]) the classes $\tilde{b}_{r}^{j}(X) \in H_{K}^{*}(M(c))$ are of the form $\tilde{b}_{r}^{j}(X)=\operatorname{pr}_{1}^{*}\left(\tilde{b}_{r}^{j}(X)\right)_{1}$ where $\left(\tilde{b}_{r}^{j}(X)\right)_{1} \in H_{K}^{*}\left(K^{2 g}\right)$ and $\operatorname{pr}_{1}: M(c) \rightarrow K^{2 g}$ is the projection in (4.4). It follows that

$$
s_{\Lambda_{0}}^{*} \tilde{b}_{r}^{j}(X)=\tilde{b}_{r}^{j}(X)
$$

and

$$
s_{\Lambda_{0}}^{*} \tilde{a}_{r}(X)=\tilde{a}_{r}(X) .
$$

Furthermore we see from (8.30) of [30] that $\tilde{f}_{2}(X)$ is of the form

$$
\tilde{f}_{2}(X)=\operatorname{pr}_{1}^{*} f_{2}^{1}+\langle\mu, X\rangle
$$

where $f_{2}^{1} \in H_{K}^{*}\left(K^{2 g}\right)$ and $\mu: M(c) \rightarrow \mathbf{k}$ is the moment map (which is the restriction to $M(c)$ of minus the projection $K^{2 g} \times \mathbf{k} \rightarrow \mathbf{k}$; see Proposition 4.1). It follows from this that for any $\Lambda_{0}$ in the integer lattice $\Lambda^{I}$ of $\mathbf{t}$ (the kernel of the exponential map),

$$
s_{\Lambda_{0}}^{*} \tilde{f}_{2}(X)=\tilde{f}_{2}(X)-\left\langle\Lambda_{0}, X\right\rangle .
$$

\section{Equivariant Poincaré duals}

We are aiming to apply nonabelian localization to the extended moduli space $M(c)$ defined in the previous section. In order to overcome the problem that $M(c)$ is singular, instead of working with integrals over $M(c)$ of equivariant differential forms, we shall integrate over $K^{2 g} \times \mathbf{k}$ after first multiplying by a suitable equivariantly closed differential form on $K^{2 g} \times \mathbf{k}$ with support near $M(c)$ which can be thought of as representing the equivariant Poincaré dual to $M(c)$ in $K^{2 g} \times \mathbf{k}$. So we need to construct such an equivariantly closed differential form.

Remark 5.1. In our earlier article [32] covering the case when the bundles have rank $n=2$, we overcame the problem of the singularities of $M(c)$ in a slightly different way, by perturbing the central constant $c \in \mathrm{SU}(n)$ to a nearby element of the maximal torus $T$. This method can be generalized to cover the cases when $n>2$, but it seems a little more straightforward to use equivariant Poincaré duals, so we adopt the latter approach here. 
Remark 5.2. Related constructions of equivariantly closed differential forms representing the Poincaré dual to a submanifold appear in the literature. ${ }^{14}$ In Kalkman's paper [34] and Mathai-Quillen's paper [40], an equivariantly closed differential form which is rapidly decreasing away from a submanifold and represents the Poincare dual to the submanifold is given; such a form is often referred to as the Thom form, as the cohomology class it represents is the Thom class of the normal bundle to the submanifold. The forms constructed in [34] and [40] are not compactly supported; a construction of a compactly supported equivariantly closed form representing the Poincaré dual of a submanifold is given in Section 2.3 of [19]. For completeness, in this section we provide a construction of an equivariantly closed form representing the Poincaré dual.

First we consider the simpler problem of constructing an equivariant Poincaré dual to the origin in a one-dimensional representation $\chi$ of a circle. If we did not need to find a form with support near the origin we could represent the equivariant Poincaré dual by $\chi$ itself, regarded as an equivariant differential form. However compact support will be important later, so we need to be a little more careful.

Lemma 5.3. Let $T=U(1)$ act on $\mathbb{C}$ via a weight $\chi: T \rightarrow U(1)$. Then we can find an equivariantly closed differential form $\alpha_{\chi} \in \Omega_{T}^{2}(\mathbb{C})$ on $\mathbb{C}$ with compact support arbitrarily close to 0 , such that

$$
\int_{\mathbb{C}} \eta \alpha_{\chi}=\left.\eta\right|_{0} \in H_{T}^{*}
$$

for all equivariantly closed forms $\eta \in \Omega_{T}^{*}(\mathbb{C})$. Moreover $\alpha_{\chi} \in \chi+D\left(\Omega_{T}^{*}(\mathbb{C})\right.$ ), so that $\alpha_{\chi}$ represents the same equivariant cohomology class on $\mathbb{C}$ as $\chi$.

Proof. Let $X^{\sharp}$ denote the vector field on $\mathbb{C}$ given by the infinitesimal action of $X \in \mathbf{t}$. There is a $T$-invariant closed differential 1-form on $\mathbb{C}-\{0\}$, given in polar coordinates $(r, \theta)$ by $\frac{d \theta}{2 \pi}$, such that $\iota_{X}\left(\frac{d \theta}{2 \pi}\right)$ is identically equal to $\chi(X)$ for every $X \in \mathbf{t}$. We can choose a smooth $T$-invariant function $b: \mathbb{C} \rightarrow[0, \infty)$ with support in an arbitrarily small neighbourhood of 0 which is identically equal to 1 on some smaller neighbourhood of 0 , and let

$$
\alpha_{\chi}(X)=\chi(X)+D\left((1-b) \frac{d \theta}{2 \pi}\right)=\chi(X)+d\left((1-b) \frac{d \theta}{2 \pi}\right)+(b-1) \chi(X)
$$

where $D$ is the equivariant differential defined at (3.2) and $d$ is the ordinary differential. Then $\alpha_{\chi}$ is equivariantly closed and is zero outside the support of $b$.

\footnotetext{
${ }^{14}$ We thank P. Paradan for pointing out that the references cited below contain such constructions.
} 
Suppose that $\eta \in \Omega_{T}^{*}(\mathbb{C})$ is equivariantly closed. We wish to show that

$$
\int_{\mathbb{C}} \eta \alpha_{\chi}=\left.\eta\right|_{0}
$$

First we shall show that the integral

$$
\int_{\mathbb{C}} \eta \alpha_{\chi}
$$

is independent of the choice of the function $b$.

If $\rho>0$ is sufficiently small and $R>0$ is sufficiently large, then $b$ is identically equal to 1 on the disc $D_{\rho}$ centre 0 and radius $\rho$, and $b$ is identically equal to 0 outside the disc $D_{R}$ centre 0 and radius $R$. Then

$$
\int_{\mathbb{C}} \eta \alpha_{\chi}=\chi \int_{D_{\rho}} \eta+\int_{D_{R}-D_{\rho}} \eta \alpha_{\chi}
$$

Now $\eta$ is a polynomial function from $\mathbf{t}$ to the ordinary de Rham complex $\Omega^{*}(\mathbb{C})$, so we can write

$$
\eta=\eta^{(0)}+\eta^{(1)}+\eta^{(2)}
$$

where $\eta^{(j)}$ is a polynomial function from $\mathbf{t}$ to $\Omega^{j}(\mathbb{C})$ for $j=0,1,2$. Similarly

$$
\alpha_{\chi}=\alpha_{\chi}^{(0)}+\alpha_{\chi}^{(1)}+\alpha_{\chi}^{(2)}
$$

where $\alpha_{\chi}^{(0)}=b \chi, \alpha_{\chi}^{(1)}=0$ and $\alpha_{\chi}^{(2)}=d\left((1-b) \frac{d \theta}{2 \pi}\right)$. Since $D \eta=d \eta-\iota_{X^{\sharp}} \eta$ is zero, we have $d \eta^{(0)}=\iota_{X}^{\sharp} \eta^{(2)}$. As any 2 -form on $\mathbb{C}$ is a $C^{\infty}$ function on $\mathbb{C}$ multiplied by the nowhere vanishing 2 -form given in polar coordinates by $\frac{r d \theta d r}{2 \pi}$, and since $\iota_{X^{\sharp}}\left(\frac{r d \theta d r}{2 \pi}\right)=\chi(X) r d r$, it follows that

$$
\chi(X) \eta^{(2)}(X)=\frac{d \theta}{2 \pi} d \eta^{(0)}(X)
$$

on $\mathbb{C}-\{0\}$ where $d \theta$ is defined. Hence

$$
\begin{aligned}
\int_{D_{R}-D_{\rho}} \eta \alpha_{\chi} & =\int_{D_{R}-D_{\rho}} \eta^{(2)} \alpha_{\chi}^{(0)}+\eta^{(0)} \alpha_{\chi}^{(2)} \\
& =\int_{D_{R}-D_{\rho}} b \frac{d \theta}{2 \pi} d \eta^{(0)}+\eta^{(0)} d\left((1-b) \frac{d \theta}{2 \pi}\right) \\
& =-\int_{D_{R}-D_{\rho}} d\left(b \eta^{(0)} \frac{d \theta}{2 \pi}\right) \\
& =\int_{\partial D_{\rho}} b \eta^{(0)} \frac{d \theta}{2 \pi}-\int_{\partial D_{R}} b \eta^{(0)} \frac{d \theta}{2 \pi} \\
& =\int_{\partial D_{\rho}} \eta^{(0)} \frac{d \theta}{2 \pi}
\end{aligned}
$$

by Strokes' theorem, since $b$ is identically one on $\partial D_{\rho}$ and identically zero on 
$\partial D_{R}$. It follows that

$$
\int_{\mathbb{C}} \eta \alpha_{\chi}=\chi \int_{D_{\rho}} \eta+\int_{\partial D_{\rho}} \eta^{(0)} \frac{d \theta}{2 \pi}
$$

is independent of the choice of $b$.

Now $\rho$ can be taken arbitrarily small, and $\chi \int_{D_{\rho}} \eta \rightarrow 0$ as $\rho \rightarrow 0$. Moreover by continuity, for fixed $X \in \mathbf{t}$ and any $\varepsilon>0$ we can choose $\rho$ so that $\eta^{(0)}$ differs from $\left.\eta^{(0)}\right|_{0}=\left.\eta\right|_{0}$ by at most $\varepsilon$ on $D_{\rho}$. Then

$$
\left|\int_{\partial D_{\rho}} \eta^{(0)} \frac{d \theta}{2 \pi}-\eta^{(0)}\right|_{0}|=| \int_{\partial D_{\rho}}\left(\eta^{(0)}-\left.\eta^{(0)}\right|_{0}\right) \frac{d \theta}{2 \pi} \mid \leq \varepsilon .
$$

Thus $\int_{\mathbb{C}} \eta \alpha_{\chi}-\left.\eta\right|_{0}$ tends to zero as $\rho$ tends to 0 . Since $\int_{\mathbb{C}} \eta \alpha_{\chi}$ and $\left.\eta\right|_{0}$ are independent of $\rho$ we deduce that

$$
\int_{\mathbb{C}} \eta \alpha_{\chi}=\left.\eta\right|_{0}
$$

as required.

Lemma 5.4. Let $T$ be a torus acting trivially on $\mathbb{R}$. Then we can find an equivariantly closed differential form $\alpha_{0} \in \Omega_{T}^{*}(\mathbb{R})$ on $\mathbb{R}$ with compact support arbitrarily close to 0 , such that

$$
\int_{\mathbb{R}} \eta \alpha_{0}=\left.\eta\right|_{0} \in H_{T}^{*}
$$

for all equivariantly closed forms $\eta \in \Omega_{T}^{*}(\mathbb{R})$.

Proof. We have $\Omega_{T}^{*}(\mathbb{R})=S\left(\mathbf{t}^{*}\right) \otimes \Omega^{*}(\mathbb{R})$ and $\eta \in S\left(\mathbf{t}^{*}\right) \otimes \Omega^{0}(\mathbb{R})$ is equivariantly closed if and only if it is constant on $\mathbb{R}$, so we can take $\alpha_{0}$ to be the standard volume form on $\mathbb{R}$ multiplied by any bump function compactly supported near 0 with unit integral.

COROllary 5.5. Let $T$ be a torus acting linearly on $\mathbb{C}^{n}$ with weights $\chi_{1}, \ldots, \chi_{n}$ and trivially on $\mathbb{R}^{m}$. Then we can find an equivariantly closed differential form $\alpha \in \Omega_{T}^{2 n}\left(\mathbb{C}^{n} \times \mathbb{R}^{m}\right)$ on $\mathbb{C}^{n} \times \mathbb{R}^{m}$ with compact support arbitrarily close to 0 , such that

$$
\int_{\mathbb{C}^{n} \times \mathbb{R}^{m}} \eta \alpha=\eta_{0} \in H_{T}^{*}
$$

for all equivariantly closed forms $\eta \in \Omega_{T}^{*}\left(\mathbb{C}^{n} \times \mathbb{R}^{m}\right)$. Moreover if $m=0$ then $\alpha \in \chi_{1} \ldots \chi_{n}+D\left(\Omega_{T}^{*}\left(\mathbb{C}^{n}\right)\right)$.

Proof. The action of $T$ on the copy of $\mathbb{C}$ in $\mathbb{C}^{n}$ on which it acts via the weight $\chi_{j}$ factors through an action of $T / \operatorname{ker} \chi_{j} \cong U(1)$ (unless $\chi_{j}=0$ in which case we can replace ker $\chi_{j}$ by any subtorus of $T$ of codimension one). We can construct $\alpha_{\chi_{j}} \in \Omega_{U(1)}^{*}(\mathbb{C})$ as in Lemma 5.3 and $m$ copies of $\alpha_{0}$ as in 
Lemma 5.4, and then define $\alpha$ to be the wedge product of the pullbacks of the $\alpha_{\chi_{j}}$ and $\alpha_{0}$ to $\Omega_{T}^{*}\left(\mathbb{C}^{n} \times \mathbb{R}^{m}\right)$ via the projections of $\mathbb{C}^{n} \times \mathbb{R}^{m}$ to $\mathbb{C}$ and $\mathbb{R}$ and the homomorphisms $T \rightarrow U(1)$ induced by the weights $\chi_{j}$.

Now we shall relax our assumption that $c$ is a central element of $K$, and assume only that $c \in T$. This will be important later when we apply induction on $n$ (see Remark 6.4 below).

Corollary 5.6. Let $T$ be the maximal torus of $K=\mathrm{SU}(n)$ acting on $K$ by conjugation. If $c \in T$ then we can find a T-equivariantly closed differential form $\alpha \in \Omega_{T}^{*}(K)$ on $K$ with support arbitrarily close to $c$ such that

$$
\int_{K} \eta \alpha=\left.\eta\right|_{c} \in H_{T}^{*}
$$

for all T-equivariantly closed differential forms $\eta \in \Omega_{T}^{*}(K)$.

Proof. There is a $T$-equivariant diffeomorphism $\phi$ from a $T$-invariant neighbourhood $U$ of 0 in the Lie algebra $\mathbf{k}$ of $K$ to a $T$-invariant neighbourhood $V$ of $c$ in $K$ given by

$$
\phi(X)=c \exp (X) .
$$

By Corollary 5.5 we can find $\tilde{\alpha} \in \Omega_{T}^{*}(\mathbf{k})$ with arbitrarily small compact support contained in $U$, such that

$$
\int_{\mathbf{k}} \eta \tilde{\alpha}=\left.\eta\right|_{0} \in H_{T}^{*}
$$

for all equivariantly closed forms $\eta \in \Omega_{T}^{*}(\mathbf{k})$. Then we can define $\alpha$ to be $\left(\phi^{-1}\right)^{*}(\tilde{\alpha})$.

\section{Note that}

$$
M(c)=\left\{\left(h_{1}, \ldots, h_{2 g}, \Lambda\right) \in K^{2 g} \times \mathbf{k}: \prod_{j=1}^{g} h_{2 j-1} h_{2 j} h_{2 j-1}^{-1} h_{2 j}^{-1}=c \exp (\Lambda)\right\}
$$

can be expressed as $M(c)=P^{-1}(c)$ where $P: K^{2 g} \times \mathbf{k} \rightarrow K$ is defined by

$$
P\left(h_{1}, \ldots, h_{2 g}, \Lambda\right)=\prod_{j=1}^{g} h_{2 j-1} h_{2 j} h_{2 j-1}^{-1} h_{2 j}^{-1} \exp (-\Lambda) .
$$

Proposition 5.7. If $T$ is the maximal torus of $K=\mathrm{SU}(n)$ and $c \in T$ then there is a T-equivariantly closed differential form $\alpha \in \Omega^{*}\left(K^{2 g} \times \mathbf{k}\right)$ of degree $n^{2}-1$ on $K^{2 g} \times \mathbf{k}$ with support contained in a neighbourhood of $M(c)$ of the form $P^{-1}(V)$ where $V$ is an arbitrarily small neighbourhood of $c$ in $K$, such that

$$
\int_{K^{2 g \times \mathbf{k}}} \eta \alpha=\left.\int_{M(c)} \eta\right|_{M(c)} \in H_{T}^{*}
$$

for any $T$-equivariantly closed form $\eta \in \Omega_{T}^{*}\left(K^{2 g} \times \mathbf{k}\right)$ for which the intersection of $P^{-1}(\bar{V})$ with the support of $\eta$ is compact. 
Proof. By Corollary 5.6 we can find a $T$-equivariantly closed differential form $\hat{\alpha} \in \Omega_{T}^{*}(K)$ on $K$ with support in $V$ such that

$$
\int_{K} \eta \hat{\alpha}=\left.\eta\right|_{c} \in H_{T}^{*}
$$

for all $T$-equivariantly closed forms $\eta \in \Omega_{T}^{*}(K)$. Let $\alpha=P^{*}(\hat{\alpha})$; by the functoriality of the equivariant pushforward map (cf. Section 3 of [3]) this has the properties we want.

Remark 5.8. In fact if $V^{\prime}$ is any neighbourhood of $c$ in $K$ containing $\bar{V}$ then we have

$$
\int_{P^{-1}\left(V^{\prime}\right)} \eta \alpha=\left.\int_{M(c)} \eta\right|_{M(c)} \in H_{T}^{*}
$$

for any $T$-equivariantly closed form $\eta \in \Omega_{T}^{*}\left(P^{-1}\left(V^{\prime}\right)\right)$ on $P^{-1}\left(V^{\prime}\right)$ for which the intersection of $P^{-1}(\bar{V})$ with the support of $\eta$ is compact.

Remark 5.9. As we are going to use Proposition 5.7 to convert integrals over $M(c)$ into integrals over $K^{2 g} \times \mathbf{k}$ (or at least over neighbourhoods of $M(c)$ in $K^{2 g} \times \mathbf{k}$ of the form $P^{-1}(V)$ for arbitrarily small neighbourhoods $V$ of $c$ in $K$ ), we shall need to be able to extend $T$-equivariant cohomology classes $\eta$ on $M(c)$ to $T$-equivariant cohomology classes on neighbourhoods of $M(c)$ in $K^{2 g} \times \mathbf{k}$ of this form $P^{-1}(V)$. This will always be possible by the continuity properties of cohomology (see e.g. [14, VIII 6.18]) because $\eta$ will always have compact support in $M(c)$; more precisely we will in fact be converting integrals over $M(c) \cap\left(K^{2 g} \times B\right)$ for compact subsets $B$ of $\mathbf{k}$ into integrals over $P^{-1}(V) \cap\left(K^{2 g} \times B\right)$.

Note that the centre $Z_{n}$ of $K=\mathrm{SU}(n)$ is a finite group of order $n$ which acts trivially on $K^{2 g} \times \mathbf{k}$.

LEMmA 5.10. Suppose that $c=\operatorname{diag}\left(c_{1}, \ldots, c_{n}\right) \in T$ is such that the product of no proper subsequence of $c_{1}, \ldots, c_{n}$ is 1 . Then the quotient $T / Z_{n}$ of $T$ by the centre $Z_{n}$ of $K=\mathrm{SU}(n)$ acts freely on $P^{-1}(V) \cap \mu^{-1}(0)$ for any sufficiently small $T$-invariant neighbourhood $V$ of $c$ in $K$.

Proof. Suppose that $T / Z_{n}$ does not act freely on $P^{-1}(V) \cap \mu^{-1}(0)$. Then there exist $t_{1}, \ldots, t_{n} \in \mathbb{C}$, not all equal, such that $t_{1} \ldots t_{n}=1$, and some element $(h, 0)=\left(h_{1}, \ldots, h_{2 g}, 0\right)$ of $P^{-1}(V) \cap \mu^{-1}(0)$ fixed by $\operatorname{diag}\left(t_{1}, \ldots, t_{n}\right)$. Then each $h_{j}$ is block diagonal with respect to the decomposition of $\{1, \ldots, n\}$ as the union of $\left\{i: t_{i}=t_{1}\right\}$ and $\left\{i: t_{i} \neq t_{1}\right\}$, which implies that

$$
P(h, 0)=\left[\begin{array}{cc}
A & 0 \\
0 & B
\end{array}\right]
$$

where $A$ and $B$ are products of commutators and hence satisfy $\operatorname{det} A=1=$ $\operatorname{det} B$. The result follows. 
Remark 5.11. It follows from this lemma that we can extend the definition of the composition

$$
\Phi: H_{T}^{*}(M(c)) \rightarrow H_{T}^{*}\left(M(c) \cap \mu^{-1}(0)\right) \cong H^{*}\left(M(c) \cap \mu^{-1}(0) / T\right)
$$

to.

$\Phi: H_{T}^{*}\left(P^{-1}(V)\right) \rightarrow H_{T}^{*}\left(P^{-1}(V) \cap \mu^{-1}(0)\right) \cong H^{*}\left(P^{-1}(V) \cap \mu^{-1}(0) / T\right)$.

By 1.18 of [23] (see Remark 3.10 above), when $T=U(1)$ is a circle then $\Phi$ is given on the level of forms by

$$
\Phi(\eta)=\operatorname{Res}_{X=0} \iota_{v}\left(\frac{\zeta \eta}{X-d \zeta}\right)
$$

where the vector field $v$ is the infinitesimal generator of the $U(1)$ action and $\zeta$ is a $U(1)$-invariant differential 1-form on $P^{-1}(V) \cap \mu^{-1}(0)$ such that $\iota_{v}(\zeta)=1$. (Strictly speaking the residue is an invariant form on $P^{-1}(V) \cap \mu^{-1}(0)$ which descends to a form on $\left(P^{-1}(V) \cap \mu^{-1}(0)\right) / T$.) Thus when $T=U(1)$,

$$
\int_{M(c) \cap \mu^{-1}(0) / T} \Phi(\eta)=\int_{M(c) \cap \mu^{-1}(0)} \operatorname{Res}_{X=0} \frac{\zeta \eta}{X-d \zeta},
$$

and it follows that if $\alpha$ is defined as in Proposition 5.7 for $n=2$ and $V^{\prime}$ is any neighbourhood of $c$ in $\mathrm{SU}(2)$ containing $\bar{V}$,

$$
\begin{array}{r}
\int_{P^{-1}\left(V^{\prime}\right) \cap \mu^{-1}(0) / T} \Phi(\eta \alpha)=\int_{P^{-1}\left(V^{\prime}\right) \cap \mu^{-1}(0)} \operatorname{Res}_{X=0} \frac{\zeta \eta \alpha}{X-d \zeta} \\
=\int_{M(c) \cap \mu^{-1}(0)} \operatorname{Res}_{X=0} \frac{\zeta \eta}{X-d \zeta}=\int_{M(c) \cap \mu^{-1}(0) / T} \Phi(\eta)
\end{array}
$$

for any $T$-equivariantly closed differential form $\eta \in \Omega_{T}^{*}\left(P^{-1}\left(V^{\prime}\right)\right)$ such that the intersection of $P^{-1}(\bar{V})$ with the support of $\eta$ is compact. Here we have used the same notation for $\eta$ and its restriction to $M(c)$.

When $n>2$, so that the maximal torus $T$ of $K=\mathrm{SU}(n)$ has dimension higher than one, then $\Phi(\eta)$ and $\int_{M(c) \cap \mu^{-1}(0) / T} \Phi(\eta)$ are given by similar formulas involving $n-1$ iterated residues (see [23]). In particular the support of $\Phi(\eta)$ is contained in the image of the support of $\eta$, and

$$
\int_{P^{-1}\left(V^{\prime}\right) \cap \mu^{-1}(0) / T} \Phi(\eta \alpha)=\int_{M(c) \cap \mu^{-1}(0) / T} \Phi(\eta)
$$

for any $T$-equivariantly closed differential form $\eta \in \Omega_{T}^{*}\left(P^{-1}\left(V^{\prime}\right)\right)$ such that the intersection of $P^{-1}(\bar{V})$ with the support of $\eta$ is compact. 


\section{Nonabelian localization applied to extended moduli spaces}

Naïve application of the residue formula (Theorem 3.1) to the extended moduli space $M(c)$, using (2.1) and Remark 4.3 and ignoring the fact that $M(c)$ is noncompact and has singularities, yields

$$
\begin{aligned}
& \prod_{r=2}^{n} a_{r}^{m_{r}} \exp \left(f_{2}\right)[\mathcal{M}(n, d)] \\
& =n C_{K} \operatorname{Res}\left(\mathcal{D}^{2}(X)\left(\int_{F} e^{\omega}\right) \sum_{\delta \in \Lambda^{I}} \frac{\prod_{r=2}^{n} \tau_{r}(-X)^{m_{r}} e^{(\tilde{c}-\delta)(X)}}{\left((-1)^{n+\mathcal{D}^{2}}(X)\right)^{g}}\right)
\end{aligned}
$$

where the constant $C_{K}$ is as defined at (3.7). The main problem with (6.1) (related to the noncompactness of $M(c)$, which permits the fixed point set $M(c)^{T}$ to be the union of infinitely many components $F_{\delta}$ ) is that the sum over $\delta$ does not converge for $X \in \mathbf{t}$. In this section we shall instead apply the version of nonabelian localization due to Guillemin-Kalkman and Martin (Propositions 3.6 and 3.9) to $M(c)$, using Remarks 3.8 and 3.10; this will lead to a proof that (6.1) is true if interpreted appropriately (see Remark 8.6). First we use Proposition 3.6.

Lemma 6.1. Let $|W|=n$ ! be the order of the Weyl group $W$ of $K=$ $\mathrm{SU}(n)$, and let $c=\operatorname{diag}\left(e^{2 \pi i d / n}, \ldots, e^{2 \pi i d / n}\right)$ where $d$ is coprime to $n$. If $V$ is a sufficiently small neighbourhood of $c$ in $K$ so that the quotient $T / Z_{n}$ of $T$ by the centre $Z_{n}$ of $K=\mathrm{SU}(n)$ acts freely on $P^{-1}(V) \cap \mu^{-1}(0)$ (see Lemma 5.10), then for any $\eta \in H_{K}^{*}(X)$,

$$
\int_{\mathcal{M}(n, d)} \Phi\left(\eta e^{\bar{\omega}}\right)=\frac{1}{|W|} \int_{N(c)} \Phi\left(\mathcal{D} \eta e^{\bar{\omega}}\right)=\frac{1}{|W|} \int_{N(V)} \Phi\left(\mathcal{D} \eta e^{\bar{\omega}} \alpha\right)
$$

where

$$
N(c)=M(c) \cap \mu^{-1}(0) / T
$$

for $\mu: K^{2 g} \times \mathbf{k} \rightarrow \mathbf{k}$ given by minus the projection onto $\mathbf{k}$ and

$$
N(V)=P^{-1}(V) \cap \mu^{-1}(0) / T .
$$

Also $\alpha$ is a T-equivariantly closed form on $K^{2 g} \times \mathbf{k}$ representing the $T$-equivariant Poincaré dual to $M(c)$, which is chosen as in Proposition 5.7 so that the support of $\alpha$ is contained in $P^{-1}(V)$ and has compact intersection with $\mu^{-1}(0)$.

Proof. Since $\mathcal{M}(n, d)=M(c) \cap \mu^{-1}(0) / K$, we can first identify $\int_{\mathcal{M}(n, d)} \Phi\left(\eta e^{\bar{\omega}}\right)$ with

$$
\frac{1}{|W|} \int_{N(c)} \Phi\left(\mathcal{D} \eta e^{\bar{\omega}}\right)
$$


via Proposition 3.6, whose proof works in this situation even though $M(c)$ is noncompact and singular, because $\mu$ is proper and $M(c)$ is nonsingular in a neighbourhood of $\mu^{-1}(0)$ (see Remark 3.8). Then we use Remark 5.11.

Next we need to summarize some conventions on the roots and weights of $\mathrm{SU}(n)$. The simple roots $\left\{e_{j}: j=1, \ldots, n-1\right\}$ of $\mathrm{SU}(n)$ are elements of $\mathbf{t}^{*} ;$ in terms of the standard identification of $\mathbf{t}$ with $\left\{\left(X_{1}, \ldots, X_{n}\right) \in \mathbb{R}^{n}: \sum_{i} X_{i}=0\right\}$ under which $\left(X_{1}, \ldots, X_{n}\right) \in \mathbb{R}^{n}$ satisfying $\sum_{i} X_{i}=0$ corresponds to $X=$ $\operatorname{diag}\left(2 \pi i X_{1}, \ldots, 2 \pi i X_{n}\right) \in \mathbf{t}$, they are given by

$$
e_{j}(X)=X_{j}-X_{j+1}
$$

The dual basis to the basis of simple roots (with respect to the inner product $\langle\cdot, \cdot\rangle$ defined at (2.2) above, which is the usual Euclidean inner product on $\left.\mathbb{R}^{n}\right)$ is the set of fundamental weights $w_{j} \in \mathbf{t}^{*}$ given by

$$
w_{j}(X)=X_{1}+\cdots+X_{j}
$$

If we use this same inner product to identify $\mathbf{t}^{*}$ with $\mathbf{t}$, the simple roots become identified with a set of generators

$$
\hat{e_{j}}=(0, \ldots, 0,1,-1,0, \ldots, 0)
$$

for the integer lattice $\Lambda^{I}$ of $\mathbf{t}$, and the fundamental weights correspond to elements $\hat{w}_{j} \in \mathbf{t}$ given by

$$
\hat{w}_{j}=(1, \ldots, 1,0, \ldots, 0)-\frac{j}{n}(1, \ldots, 1) .
$$

In particular we have

$$
\hat{w}_{n-1}=\frac{1}{n}(1, \ldots, 1,-(n-1)) .
$$

Since we shall later apply induction on $n$, it will be convenient to label certain spaces, groups and Lie algebras by the associated value of $n$. In particular the space $M(c)$ will sometimes be denoted by $M_{n}(c)$, the maximal torus $T$ of $\operatorname{SU}(n)$ by $T_{n}$, its Lie algebra $\mathbf{t}$ by $\mathbf{t}_{n}$, and the map $\Phi$ by $\Phi_{n}$.

We define a one-dimensional torus $\hat{T}_{1} \cong S^{1}$ in $\mathrm{SU}(n)$ generated by $\hat{e_{1}}$ : it is identified with $S^{1}$ via

$$
t \in S^{1} \mapsto\left(t, t^{-1}, 1, \ldots, 1\right) \in \hat{T}_{1} .
$$

The (one-dimensional) Lie algebra $\hat{\mathbf{t}}_{1}$ is spanned by $\hat{e_{1}}$. Its orthocomplement in $\mathbf{t}$ is

$$
\mathbf{t}_{n-1}=\left\{\left(X_{1}, \ldots, X_{n}\right) \in \mathbb{R}^{n}: X_{1}=X_{2}, \sum_{j=1}^{n} X_{j}=0\right\}
$$


Define $T_{n-1}$ to be the torus given by $\exp \left(\mathbf{t}_{n-1}\right)$ :

$$
T_{n-1}=\left\{\left(t_{1}, t_{1}, t_{3} \ldots, t_{n-1}, t_{n}\right) \in U(1)^{n}:\left(t_{1}\right)^{2}\left(\prod_{j=3}^{n} t_{j}\right)=1\right\}
$$

then $T_{n-1}$ is isomorphic to the maximal torus of SU $(n-1)$ (i.e. $T_{n-1} \cong\left(S^{1}\right)^{n-2}$ ) so this does not conflict with the notation already adopted.

Remark 6.2. The multiplication map $\hat{T}_{1} \times T_{n-1} \rightarrow \hat{T}_{1} T_{n-1}=T_{n}$ is a covering map with fibre $\hat{T}_{1} \cap T_{n-1}=\mathbb{Z}_{2}=\left\{\left(t, t^{-1}, 1, \ldots, 1\right): t=t^{-1}\right\}$.

There is the following decomposition of the ring homomorphism $\Phi_{n}$.

Proposition 6.3. For any symplectic manifold $M$ equipped with a Hamiltonian action of $T_{n}$ such that $T_{n-1}$ acts locally freely on $\mu_{T_{n-1}}^{-1}(0)$, the symplectic quotient $\mu_{T_{n}}^{-1}(0) / T_{n}$ may be identified with the symplectic quotient of $\mu_{T_{n-1}}^{-1}(0) / T_{n-1}$ by the induced Hamiltonian action of $\hat{T}_{1}$. Moreover if in addition $T_{n}$ acts locally freely on $\mu_{T_{n}}^{-1}(0)$ then the ring homomorphism $\Phi_{n}: H_{T_{n}}^{*}(M)$ $\rightarrow H^{*}\left(\mu_{T_{n}}^{-1}(0) / T_{n}\right)$ factors as

$$
\Phi_{n}=\hat{\Phi}_{1} \circ \Phi_{n-1}
$$

where

$$
\begin{aligned}
\Phi_{n-1}: H_{T_{n}}^{*}(M) \rightarrow H_{T_{n}}^{*}\left(\mu_{T_{n-1}}^{-1}(0)\right) & \cong H_{\hat{T}_{1} \times T_{n-1}}^{*}\left(\mu_{T_{n-1}}^{-1}(0)\right) \\
& \cong H_{\hat{T}_{1}}^{*}\left(\mu_{T_{n-1}}^{-1}(0) / T_{n-1}\right)
\end{aligned}
$$

and

$$
\begin{aligned}
\hat{\Phi}_{1}: H_{\hat{T}_{1}}^{*}\left(\mu_{T_{n-1}}^{-1}(0) / T_{n-1}\right) & \rightarrow H^{*}\left(\left(\mu_{T_{n-1}}^{-1}(0) \cap \mu_{\hat{T}_{1}}^{-1}(0) / T_{n-1} \times \hat{T}_{1}\right)\right. \\
& \cong H^{*}\left(\mu_{T_{n}}^{-1}(0) / T_{n}\right)
\end{aligned}
$$

Proof. The isomorphisms

$$
H_{T_{n}}^{*}\left(\mu_{T_{n-1}}^{-1}(0)\right) \cong H_{\hat{T}_{1} \times T_{n-1}}^{*}\left(\mu_{T_{n-1}}^{-1}(0)\right) \cong H_{\hat{T}_{1}}^{*}\left(\mu_{T_{n-1}}^{-1}(0) / T_{n-1}\right)
$$

follow from Remark 6.2 and the fact that the cohomology with complex coefficients of the classifying space of a finite group is trivial.

Since $\mu_{T_{n}}$ is a $T_{n}$-invariant map, its projection $\mu_{\hat{T}_{1}}$ onto $\hat{\mathbf{t}}_{1}$ descends to $\mu_{T_{n-1}}^{-1}(0) / T_{n-1}$ and defines a moment map for the induced $\hat{T}_{1}$-action with respect to the induced symplectic structure on $\mu_{T_{n-1}}^{-1}(0) / T_{n-1}$. The rest then follows from Remark 6.2 and naturality (cf. [23], after (2.9)).

Remark 6.4. From now on, thanks to Lemma 6.1, we shall be working with quotients by $T$ and subgroups of $T$, rather than quotients by $K$. Because of this our arguments will apply to $M(c)$ when $c$ belongs to $T$ but is no longer 
necessarily a central element of $K$. This will be important later, when we apply induction on $n$ using Proposition 6.3. The only condition we will need to impose on $c \in T$ is that $c=\operatorname{diag}\left(c_{1}, \ldots, c_{n}\right)$ where the product of no proper subsequence of $\left(c_{1}, \ldots, c_{n}\right)$ is 1 ; this is certainly true for our original choice of $c$ when $c_{j}=e^{2 \pi i d / n}$ for all $j$ with $d$ coprime to $n$.

So for any $c \in T$, let us define

$$
\begin{aligned}
M(c) & =M_{n}(c)=P^{-1}(c) \\
& =\left\{\left(h_{1}, \ldots, h_{2 g}, \Lambda\right) \in K^{2 g} \times \mathbf{k}: \prod_{j=1}^{g} h_{2 j-1} h_{2 j} h_{2 j-1}^{-1} h_{2 j}^{-1}=c \exp (\Lambda)\right\}
\end{aligned}
$$

where $P: K^{2 g} \times \mathbf{k} \rightarrow K$ is defined by

$$
P\left(h_{1}, \ldots, h_{2 g}, \Lambda\right)=\prod_{j=1}^{g} h_{2 j-1} h_{2 j} h_{2 j-1}^{-1} h_{2 j}^{-1} \exp (-\Lambda) .
$$

Let us also define

$$
N_{n}(c)=M(c) \cap \mu^{-1}(0) / T_{n}
$$

and

$$
N_{n}(V)=P^{-1}(V) \cap \mu^{-1}(0) / T_{n}
$$

where $V$ is a small $T$-invariant neighbourhood of $c$ in $K$.

Proposition 6.5. Suppose $c=\operatorname{diag}\left(c_{1}, \ldots, c_{n}\right) \in T$ is such that the product of no proper subset of $\left(c_{1}, \ldots, c_{n}\right)$ is 1 . Then the group $T_{n-1} / \mathbb{Z}_{n}$, where $\mathbb{Z}_{n}$ consists of the identity matrix multiplied by $n^{\text {th }}$ roots of unity, acts freely on $P^{-1}(V) \cap \mu_{T_{n-1}}^{-1}(0)$ for any sufficiently small $T$-invariant neighbourhood $V$ of $c$ in $K$. Hence the quotient $P^{-1}(V) \cap \mu_{T_{n-1}}^{-1}(0) / T_{n-1}$ is smooth.

Proof. The conjugation action of $\left(t_{1}, \ldots, t_{n}\right) \in U(1)^{n}$ on the space of $n \times n$ matrices sends

$$
\left(A_{i j}\right) \mapsto\left(t_{i} t_{j}^{-1} A_{i j}\right) .
$$

Clearly $\mathbb{Z}_{n}$ acts trivially. Let us assume that $(h, \Lambda) \in M(c) \cap \mu_{T_{n-1}}^{-1}(0)$ is fixed by the action of some element of $T_{n-1}$ which is not in $\mathbb{Z}_{n}$. After rearranging the coordinates $X_{3}, \ldots, X_{n}$ if necessary, we may assume that there is some $k$ between 3 and $n$ such that this element of $T_{n-1}$ is of the form $\left(t_{1}, t_{1}, t_{3}, \ldots, t_{n-1}, t_{n}\right)$ where $t_{i}=t_{1}$ if and only if $i \leq k$. Then each $h_{j}$ is block diagonal of the form

$$
\left[\begin{array}{cc}
h_{j}^{1} & 0 \\
0 & h_{j}^{2}
\end{array}\right]
$$


where $h_{j}^{1}$ is a $k \times k$ matrix and $h_{j}^{2}$ is $(n-k) \times(n-k)$. As the determinant of any commutator is one, it follows that $\prod_{j=1}^{n}\left[h_{2 j-1}, h_{2 j}\right]$ is block diagonal of the form

$$
\left[\begin{array}{cc}
A & 0 \\
0 & B
\end{array}\right]
$$

where $\operatorname{det} A=\operatorname{det} B=1$. But $\Lambda$ is also block diagonal of the same form

$$
\left[\begin{array}{cc}
\Lambda_{1} & 0 \\
0 & \Lambda_{2}
\end{array}\right]
$$

and since $(h, \Lambda) \in \mu_{T_{n-1}}^{-1}(0)$ the diagonal entries of $\Lambda$ are $(2 \pi i \lambda,-2 \pi i \lambda, 0, \ldots, 0)$ for some $\lambda \in \mathbb{R}$. Thus as $k \geq 3$ both $\Lambda_{1}$ and $\Lambda_{2}$ have trace 0 , so $\operatorname{det} \exp \Lambda_{1}=$ $1=\operatorname{det} \exp \Lambda_{2}$. Since $(h, \Lambda) \in M(c)$ it follows that the matrix $A$ must equal

$$
\operatorname{diag}\left(c_{1}, \ldots, c_{k}\right) \exp \Lambda_{1},
$$

and hence

$$
c_{1} \ldots c_{k}=\operatorname{det} A=1 \text {. }
$$

This contradiction to the hypotheses on $c$ shows that $T_{n-1} / \mathbb{Z}_{n}$ acts freely on $M(c) \cap \mu_{T_{n-1}}^{-1}(0)$, and the same argument shows that $T_{n-1} / \mathbb{Z}_{n}$ acts freely on $P^{-1}(V) \cap \mu_{T_{n-1}}^{-1}(\hat{V})$ for any sufficiently small $T$-invariant neighbourhood $V$ of $c$ in $K$ and any sufficiently small neighbourhood $\hat{V}$ of 0 in $\mathbf{t}_{n-1}$. The result follows.

Definition 6.6. There are coordinates

$$
Y_{k}=e_{k}(X)=\left\langle\hat{e_{k}}, X\right\rangle
$$

on $\mathbf{t}$, corresponding to the simple roots $e_{k} \in \mathbf{t}^{*}$.

We are now in a position to exploit Proposition 3.9 and Remark 3.10, by using the translation map $s_{\Lambda_{0}}$ defined by Lemma 4.5 , where $\Lambda_{0}=\hat{e_{1}}$ lies in the integer lattice $\Lambda^{I}$ and so satisfies $\exp \left(\Lambda_{0}\right)=1$.

LEMmA 6.7. Suppose $c=\operatorname{diag}\left(c_{1}, \ldots, c_{n}\right) \in T$ is such that the product of no proper subset of $\left(c_{1}, \ldots, c_{n}\right)$ is 1 . Suppose also that $\eta$ is a polynomial in the $\tilde{a}_{r}(X)$ and $\tilde{b}_{r}^{j}(X)$, so that $s_{\hat{e_{1}}}^{*} \eta=\eta$. If $V$ is a sufficiently small $T$ invariant neighbourhood of $c$ in $K$ so that $P^{-1}(V) \cap \mu^{-1}\left(\hat{\mathbf{t}}_{1}\right) / T_{n-1}$ is smooth (see Proposition 6.5), and if $N_{n}(V)=P^{-1}(V) \cap \mu^{-1}(0) / T_{n}$ as before, then

$$
\begin{aligned}
\int_{N_{n}(V)} \Phi_{n}\left(\eta e^{\bar{\omega}} e^{-Y_{1}} \alpha\right)= & \int_{P^{-1}(V) \cap \mu^{-1}\left(-\hat{e_{1}}\right) / T_{n}} \Phi_{n}\left(\eta e^{\bar{\omega}} \alpha\right) \\
= & \int_{N_{n}(V)} \Phi_{n}\left(\eta e^{\bar{\omega}} \alpha\right)-n_{0} \\
& \times \sum_{F \in \mathcal{F}:-\left\|\hat{\hat{e}_{1}}\right\|^{2}<\left\langle\hat{e_{1}}, \mu(F)\right\rangle<0} \operatorname{Res}_{Y_{1}=0} \int_{F} \frac{\Phi_{n-1}\left(\eta e^{\bar{\omega}} \alpha\right)}{e_{F}}
\end{aligned}
$$


where $\mathcal{F}$ is the set of components of the fixed point set of the action of $\hat{T}_{1}$ on $P^{-1}(V) \cap \mu^{-1}\left(\hat{\mathbf{t}}_{1}\right) / T_{n-1}$, and $e_{F}$ denotes the $\hat{T}_{1}$-equivariant Euler class of the normal to $F$ in $P^{-1}(V) \cap \mu^{-1}\left(\hat{\mathbf{t}}_{1}\right) / T_{n-1}$ for any $F \in \mathcal{F}$, while $n_{0}$ is the order of the subgroup of $\hat{T}_{1} / \hat{T}_{1} \cap T_{n-1}$ that acts trivially on $P^{-1}(V) \cap \mu^{-1}\left(\hat{\mathbf{t}}_{1}\right) / T_{n-1}$. Also $\alpha$ is the $T$-equivariantly closed differential form on $K^{2 g} \times \mathbf{k}$ given by Proposition 5.7 which represents the equivariant Poincaré dual of $M(c)$, chosen so that the support of $\alpha$ is contained in $P^{-1}(V)$.

Proof. Since $\mu^{-1}\left(\hat{\mathbf{t}}_{1}\right)=K^{2 g} \times \hat{\mathbf{t}}_{1}$ is contained in $\mu_{T_{n-1}}^{-1}(0)$, it follows from Proposition 6.5 that if $V$ is a sufficiently small $T$-invariant neighbourhood of $c$ in $K$, then $T_{n-1} / \mathbb{Z}_{n}$ acts freely on $P^{-1}(V) \cap \mu^{-1}\left(\hat{\mathbf{t}}_{1}\right)$ and so the quotient $P^{-1}(V) \cap \mu^{-1}\left(\hat{\mathbf{t}}_{1}\right) / T_{n-1}$ is smooth.

Since the restriction of $\mu_{\hat{T}_{1}}$ to $\mu^{-1}\left(\hat{\mathbf{t}}_{1}\right)$ is proper, and the support of $\alpha$ is contained in $P^{-1}(V)$, by Remark 3.10, Guillemin and Kalkman's proof of Proposition 3.9 can be applied to the $\hat{T}_{1}$-invariant function induced by $\mu_{\hat{T}_{1}}$ on the smooth manifold $P^{-1}(V) \cap \mu^{-1}\left(\hat{\mathbf{t}}_{1}\right) / T_{n-1}$ and the $\hat{T}_{1}$-equivariant form induced by $\eta e^{\bar{\omega}} \alpha$. In fact since $\hat{T}_{1} \cap T_{n-1} \cong \mathbb{Z}_{2}$ acts trivially we can work with the action of $\hat{T}_{1} / \hat{T}_{1} \cap T_{n-1}$ instead of the action of $\hat{T}_{1}$ (the Lie algebra and moment map are of course the same). This fits better with the choice of coordinates $Y_{k}$ defined by the simple roots $\hat{e}_{k}$ because the simple root $\hat{e}_{1}$ takes $\left(t, t^{-1}, 1, \ldots, 1\right) \in \hat{T}_{1}$ to $t^{2}$ and thus induces an isomorphism from $\hat{T}_{1} / \hat{T}_{1} \cap T_{n-1}$ to $S^{1}$. By combining this with Proposition 6.3 we get

$$
\begin{array}{r}
\int_{P^{-1}(V) \cap \mu^{-1}(0) / T_{n}} \Phi_{n}\left(\eta e^{\bar{\omega}} \alpha\right)-\int_{P^{-1}(V) \cap \mu^{-1}\left(-\hat{e_{1}}\right) / T_{n}} \Phi_{n}\left(\eta e^{\bar{\omega}} \alpha\right) \\
=n_{0} \operatorname{Res}_{Y_{1}=0} \sum_{F \in \mathcal{F}:-\left\|\hat{\hat{e}_{1}}\right\|^{2}<\left\langle\hat{e_{1}}, \mu(F)\right\rangle<0} \int_{F} \frac{\Phi_{n-1}\left(\eta e^{\bar{\omega}} \alpha\right)}{e_{F}} .
\end{array}
$$

Now note that the restriction of $P: K^{2 g} \times \mathbf{k} \rightarrow K$ to $\mu^{-1}(\mathbf{t})=K^{2 g} \times \mathbf{t}$ is invariant under the translation $s_{\Lambda_{0}}$ for $\Lambda_{0} \in \Lambda^{I}$. Therefore by construction the restriction of $\alpha$ to $\mu^{-1}(\mathbf{t})$ is also invariant under this translation. Thus by (4.9) and Definition 6.6

$\int_{P^{-1}(V) \cap \mu^{-1}\left(-\hat{e_{1}}\right) / T_{n}} \Phi_{n}\left(\eta e^{\bar{\omega}} \alpha\right)=\int_{N_{n}(V)} \Phi_{n}\left(s_{\hat{e}_{1}}^{*}\left(\eta e^{\bar{\omega}} \alpha\right)\right)=\int_{N_{n}(V)} \Phi_{n}\left(\eta e^{\bar{\omega}} e^{-Y_{1}} \alpha\right)$.

The result follows.

Remark 6.8. It will follow from the proof of Proposition 7.1 below that $n_{0}=1$ here (see Remark 7.2). 


\section{Fixed point sets of the circle action}

In this section we shall consider the components $F \in \mathcal{F}$ of the fixed point set of the action of $\hat{T}_{1}$ on the quotient $P^{-1}(V) \cap \mu^{-1}\left(\hat{\mathbf{t}}_{1}\right) / T_{n-1}$ (which appeared in Lemma 6.7). Since $P^{-1}(c)=M(c)$ and $V$ is an arbitrarily small $T$ invariant neighbourhood of $c$ in $K$, we may assume that every $F \in \mathcal{F}$ contains a component of the fixed point set of the action of $\hat{T}_{1}$ on $M(c) \cap \mu^{-1}\left(\hat{\mathbf{t}}_{1}\right) / T_{n-1}$, and each of these components is contained in a unique $F \in \mathcal{F}$. So we shall start by analysing the components of the fixed point set of the action of $\hat{T}_{1}$ on $M(c) \cap \mu^{-1}\left(\hat{\mathbf{t}}_{1}\right) / T_{n-1}$. We shall find that they can be described inductively in terms of products of spaces of the form $N(c)$ (see Remark 6.4) for smaller values of $n$. This will enable us to use induction in the next two sections to express the intersection pairings $\int_{\mathcal{M}(n, d)} \Phi\left(\eta e^{\bar{\omega}}\right)$ on the moduli spaces $\mathcal{M}(n, d)$ as iterated residues (see Theorem 8.1 and Theorem 9.12).

Proposition 7.1. Suppose that $c=\operatorname{diag}\left(c_{1}, \ldots, c_{n}\right) \in \mathrm{SU}(n)$ is such that the product of no proper subsequence of $\left(c_{1}, \ldots, c_{n}\right)$ is 1 . Then the components of the fixed point set of the action of $\hat{T}_{1}$ on the quotient $\left(M(c) \cap \mu^{-1}\left(\hat{\mathbf{t}}_{1}\right)\right) / T_{n-1}$ may be described as follows. For any subset $I$ of $\{3, \ldots, n\}$ let $I_{1}=I \cup\{1\}$ and let $I_{2}=\{1, \ldots, n\}-I_{1}$. Let $H_{I}$ be the subgroup of $\mathrm{SU}(n)$ given by

$$
H_{I}=\left\{\left(a_{i j}\right) \in \mathrm{SU}(n): a_{i j}=0 \text { if }(i, j) \in\left(I_{1} \times I_{2}\right) \cup\left(I_{2} \times I_{1}\right)\right\} .
$$

Suppose that $\lambda \in \mathbb{R}$ is a solution of

$$
e^{-2 \pi i \lambda}=c_{i_{1}} \ldots c_{i_{r}}=\prod_{j \in I_{1}} c_{j}
$$

where $r$ is the number of elements of $I_{1}=\left\{i_{1}, \ldots, i_{r}\right\}$, so that

$$
e^{2 \pi i \lambda}=\prod_{j \in I_{2}} c_{j}
$$

Then there is a component of the fixed point set given by $F_{I, \lambda}=\tilde{F}_{I, \lambda} / T_{n-1}$ where

$$
\tilde{F}_{I, \lambda}=M(c) \cap\left(H_{I}^{2 g} \times\left\{\lambda \hat{e}_{1}\right\}\right),
$$

and every component is of this form for some subset I of $\{3, \ldots, n\}$ and solution $\lambda$ to the equation above.

Proof. Suppose the $\hat{T}_{1}$ orbit of a point $\left(h_{1}, \ldots, h_{2 g}, \Lambda\right) \in \mathrm{SU}(n)^{2 g} \times \hat{\mathbf{t}}_{1}$ is contained in its orbit under $T_{n-1}$. A general element of the $\hat{T}_{1}$ orbit of an $n \times n$ 
matrix $A=\left(a_{i j}\right)$ under conjugation looks like

$$
\left[\begin{array}{lccrr}
a_{11} & t^{2} a_{12} & t a_{13} & \ldots & t a_{1 n} \\
t^{-2} a_{21} & a_{22} & t^{-1} a_{23} & \ldots & t^{-1} a_{2 n} \\
t^{-1} a_{31} & t a_{32} & a_{33} & \ldots & a_{3 n} \\
\vdots & \vdots & \vdots & \ldots & \vdots \\
t^{-1} a_{n 1} & t a_{n 2} & a_{n 3} & \ldots & a_{n n}
\end{array}\right]
$$

while a general element of the $T_{n-1}$ orbit of $A$ looks like

$$
\left[\begin{array}{lcclr}
a_{11} & a_{12} & t_{1} t_{3}^{-1} a_{13} & \ldots & t_{1} t_{n}^{-1} a_{1 n} \\
a_{21} & a_{22} & t_{1} t_{3}^{-1} a_{23} & \ldots & t_{1} t_{n}^{-1} a_{2 n} \\
t_{3} t_{1}^{-1} a_{31} & t_{3} t_{1}^{-1} a_{32} & a_{33} & \ldots & t_{3} t_{n}^{-1} a_{3 n} \\
\vdots & \vdots & \vdots & \ldots & \vdots \\
t_{n} t_{1}^{-1} a_{n 1} & t_{n} t_{1}^{-1} a_{n 2} & t_{n} t_{3}^{-1} a_{n 3} & \ldots & a_{n n}
\end{array}\right]
$$

For each $t$ there exist $t_{1}, t_{3}, \ldots, t_{n}$ such that these two matrices are equal when $A$ is any of $h_{1}, \ldots, h_{2 g}$ and $\Lambda$. Choose $t \neq t^{-1}$ and let $I$ denote the set of $j$ in $\{3, \ldots, n\}$ for which $t_{1} t_{j}^{-1}=t$. Similarly, define $J$ to be the set of $j$ in $\{3, \ldots, n\}$ for which $t_{1} t_{j}^{-1}=t^{-1}$, and let $K=\{3, \ldots, n\}-I-J$. Reordering the coordinates one finds that all the $h_{j}$ and $\Lambda$ are block diagonal where the blocks correspond to $I \cup\{1\}, J \cup\{2\}$ and $K$. Conversely, if all the $h_{j}$ are block diagonal of this form and $\Lambda \in \hat{\mathbf{t}}_{1}$, then the $\hat{T}_{1}$ orbit of $\left(h_{1}, \ldots, h_{2 g}, \Lambda\right)$ is contained in its $T_{n-1}$ orbit since given any $t \in U(1)$ we can find $\left(t_{1}, t_{1}, t_{3}, \ldots, t_{n}\right)$ in $T_{n-1}$ satisfying $t_{1} t_{j}^{-1}=t$ if $j \in I$ and $t_{1} t_{j}^{-1}=t^{-1}$ if $j \in J$.

We next prove that $K$ is empty. Suppose otherwise; then as the determinant of any commutator is one, $\operatorname{det} \prod_{j=1}^{g}\left[h_{2 j-1}^{[K]}, h_{2 j}^{[K]}\right]=1$ (where the superscript $[K]$ denotes the block of the matrix corresponding to $K$ ). Thus the $K$ block in $c$ also has determinant 1. This is impossible by the hypothesis on $c$.

Suppose now that $\left(h_{1}, \ldots, h_{2 g}, \Lambda\right) \in M(c) \cap \mu^{-1}\left(\hat{\mathbf{t}}_{1}\right)$ lies in $H_{I}^{2 g} \times \hat{\mathbf{t}}_{1}$. Then

$$
\Lambda=\lambda \hat{e_{1}}=2 \pi i \operatorname{diag}(\lambda,-\lambda, 0, \ldots, 0)
$$

for some $\lambda \in \mathbb{R}$, so the blocks $\Lambda^{\left[I_{1}\right]}$ and $\Lambda^{\left[I_{2}\right]}$ of $\Lambda$ corresponding to $I_{1}=I \cup\{1\}$ and $I_{2}=J \cup\{2\}$ satisfy $\operatorname{det} \exp \Lambda^{\left[I_{1}\right]}=e^{2 \pi i \lambda}$ and $\operatorname{det} \exp \Lambda^{\left[I_{2}\right]}=e^{-2 \pi i \lambda}$. But

$$
\operatorname{det}\left(\prod_{j=1}^{g}\left[h_{2 j-1}^{\left[I_{1}\right]}, h_{2 j}^{\left[I_{1}\right]}\right]\right)=1=\operatorname{det}\left(\prod_{j=1}^{g}\left[h_{2 j-1}^{\left[I_{2}\right]}, h_{2 j}^{\left[I_{2}\right]}\right]\right)
$$

because the determinant of any commutator is one. It therefore follows from the definition of $M(c)$ that

$$
e^{-2 \pi i \lambda}=\prod_{j \in I_{1}} c_{j}
$$

This is enough to complete the proof. 
Remark 7.2. The proof of this proposition shows that the elements of $\hat{T}_{1}$ which act trivially on the quotient $\left(M(c) \cap \mu^{-1}\left(\hat{\mathbf{t}}_{1}\right)\right) / T_{n-1}$ are precisely those represented by $t$ satisfying $t=t^{-1}$; i.e., $t= \pm 1$, or equivalently those in $\hat{T}_{1} \cap T_{n-1}$. Thus the size $n_{0}$ of the subgroup of $\hat{T}_{1} / \hat{T}_{1} \cap T_{n-1}$ acting trivially on the quotient $\left.M(c) \cap \mu^{-1}\left(\hat{\mathbf{t}}_{1}\right)\right) / T_{n-1}$ is 1 (cf. Lemma 6.7).

Proposition 7.3. Suppose that $c=\operatorname{diag}\left(c_{1}, \ldots, c_{n}\right) \in \mathrm{SU}(n)$ is such that the product of no proper subsequence of $\left(c_{1}, \ldots, c_{n}\right)$ is 1 . Suppose that $I$ is a subset of $\{3, \ldots, n\}$ with $r-1$ elements where $1 \leq r \leq n-1$, and let $I_{1}=$ $I \cup\{1\}=\left\{i_{1}, \ldots, i_{r}\right\}$ and $I_{2}=\{1, \ldots, n\}-I_{1}=\left\{i_{r+1}, \ldots, i_{n}\right\}$. Suppose also that $\lambda \in \mathbb{R}$ is a solution of

$$
e^{-2 \pi i \lambda}=\prod_{j \in I_{1}} c_{j}
$$

so that $e^{2 \pi i \lambda}=\prod_{j \in I_{2}} c_{j}$. Let

$$
c\left(I_{1}, \lambda\right)=\operatorname{diag}\left(c_{i_{1}}^{I, \lambda}, \ldots, c_{i_{r}}^{I, \lambda}\right)
$$

and

$$
c\left(I_{2},-\lambda\right)=\operatorname{diag}\left(c_{i_{r+1}}^{I, \lambda}, \ldots, c_{i_{n}}^{I, \lambda}\right)
$$

where $c_{j}^{I, \lambda}=c_{j}$ if $j \geq 3$, while $c_{1}^{I, \lambda}=c_{1} e^{2 \pi i \lambda}$ and $c_{2}^{I, \lambda}=c_{2} e^{-2 \pi i \lambda}$. Let $F_{I, \lambda}$ be defined as in Proposition 7.1. Then there is a finite to one $\left(\right.$ in fact $(r(n-r))^{2 g}$ to one) surjective smooth map

$$
\Psi_{I, \lambda}:\left(S^{1}\right)^{2 g} \times N_{r}\left(c\left(I_{1}, \lambda\right)\right) \times N_{n-r}\left(c\left(I_{2},-\lambda\right)\right) \rightarrow F_{I, \lambda} .
$$

Proof. We define a homomorphism

$$
\rho_{I}: S^{1} \times \mathrm{SU}(r) \times \mathrm{SU}(n-r) \rightarrow H_{I} \subset \mathrm{SU}(n)
$$

given by

$$
\rho_{I}:(s, A, B) \mapsto\left[\begin{array}{cc}
s^{n-r} A & 0 \\
0 & s^{-r} B
\end{array}\right]
$$

with respect to the decomposition of $\{1, \ldots, n\}$ as $I_{1} \cup I_{2}$. Note that $\rho_{I}$ restricts to an $r(n-r)$-to-one surjective homomorphism

$$
\rho_{I}: S^{1} \times T_{r} \times T_{n-r} \rightarrow T_{n} .
$$
then

$$
\text { If } \varepsilon_{r}: \mathrm{SU}(r)^{2 g} \rightarrow \mathrm{SU}(r) \text { is defined by } \varepsilon_{r}\left(h_{1}, \ldots, h_{2 g}\right)=\prod_{j=1}^{g}\left[h_{2 j-1}, h_{2 j}\right]
$$

$$
\begin{aligned}
& \varepsilon_{n}\left(\rho_{I}\left(s_{1}, A_{1}, B_{1}\right), \ldots, \rho_{I}\left(s_{2 g}, A_{2 g}, B_{2 g}\right)\right) \\
& \quad=\left[\begin{array}{cc}
\varepsilon_{r}\left(A_{1}, \ldots, A_{2 g}\right) & 0 \\
0 & \varepsilon_{n-r}\left(B_{1}, \ldots, B_{2 g}\right)
\end{array}\right] .
\end{aligned}
$$


Let us define a map

$$
\Psi_{I, \lambda}:\left(S^{1}\right)^{2 g} \times N_{r}\left(c\left(I_{1}, \lambda\right)\right) \times N_{n-r}\left(c\left(I_{2},-\lambda\right)\right) \rightarrow F_{I, \lambda}
$$

as the quotient of

$$
\begin{aligned}
\tilde{\Psi}_{I, \lambda}: & \left(S^{1}\right)^{2 g} \times\left(\mu_{\mathrm{SU}(r)}^{-1}(0) \cap M_{r}\left(c\left(I_{1}, \lambda\right)\right)\right) \\
& \times\left(\mu_{\mathrm{SU}(n-r)}^{-1}(0) \cap M_{n-r}\left(c\left(I_{2},-\lambda\right)\right)\right) \rightarrow \tilde{F}_{I, \lambda}
\end{aligned}
$$

defined by

$$
\begin{aligned}
\tilde{\Psi}_{I, \lambda}( & \left.\left(s_{1}, \ldots, s_{2 g}\right),\left(h_{1}^{\left[I_{1}\right]}, \ldots, h_{2 g}^{\left[I_{1}\right]}, 0\right),\left(h_{1}^{\left[I_{2}\right]}, \ldots, h_{2 g}^{\left[I_{2}\right]}, 0\right)\right) \\
= & \left(\rho_{I}\left(s_{1}, h_{1}^{\left[I_{1}\right]}, h_{1}^{\left[I_{2}\right]}\right), \rho_{I}\left(s_{2}, h_{2}^{\left[I_{1}\right]}, h_{2}^{\left[I_{2}\right]}\right), \ldots, \rho_{I}\left(s_{2 g}, h_{2 g}^{\left[I_{1}\right]}, h_{2 g}^{\left[I_{2}\right]}\right),\right. \\
& 2 \pi i \operatorname{diag}(\lambda,-\lambda, 0, \ldots, 0)) .
\end{aligned}
$$

Here, $\tilde{F}_{I, \lambda}$ is as defined in Proposition 7.1.

We must check that the image of $\tilde{\Psi}_{I, \lambda}$ is contained in $\tilde{F}_{I, \lambda}$. We have

$$
\varepsilon_{r}\left(h_{1}^{\left[I_{1}\right]}, \ldots, h_{2 g}^{\left[I_{1}\right]}\right)=\operatorname{diag}\left(c_{i_{1}}^{I, \lambda}, \ldots, c_{i_{r}}^{I, \lambda}\right)=c\left(I_{1}, \lambda\right)
$$

and

$$
\varepsilon_{n-r}\left(h_{1}^{\left[I_{2}\right]}, \ldots, h_{2 g}^{\left[I_{2}\right]}\right)=\operatorname{diag}\left(c_{i_{r+1}}^{I, \lambda}, \ldots, c_{i_{n}}^{I, \lambda}\right)=c\left(I_{2},-\lambda\right)
$$

In order to show that $\tilde{\Psi}_{I, \lambda}\left(\left(s_{1}, \ldots, s_{2 g}\right),\left(h_{1}^{\left[I_{1}\right]}, \ldots, h_{2 g}^{\left[I_{1}\right]}, 0\right),\left(h_{1}^{\left[I_{2}\right]}, \ldots, h_{2 g}^{\left[I_{2}\right]}, 0\right)\right)$ lies in $\tilde{F}_{I, \lambda}$ we need to check that if $\Lambda=2 \pi i \operatorname{diag}(\lambda,-\lambda, 0, \ldots, 0)$ then $c \exp (\Lambda)$ is block diagonal of the form

$$
\left[\begin{array}{cc}
c\left(I_{1}, \lambda\right) & 0 \\
0 & c\left(I_{2},-\lambda\right)
\end{array}\right]
$$

with respect to the decomposition of $\{1, \ldots, n\}$ as $I_{1} \cup I_{2}$. This follows by the choice of $c_{1}^{I, \lambda}, \ldots, c_{n}^{I, \lambda}$.

We must also check that the map $\Psi_{I, \lambda}$ is well defined on the quotient by the action of $T_{r} \times T_{n-r}$; in other words we must check that for any $t=\left(t_{1}, \ldots, t_{n}\right)$ $\in U(1)^{n}$ satisfying $t_{i_{1}}, \ldots, t_{i_{r}}=t_{i_{r+1}}, \ldots, t_{i_{n}}=1$ so that $t_{I_{1}}=\left(t_{i_{1}} \ldots t_{i_{r}}\right) \in T_{r}$ and $t_{I_{2}}=\left(t_{i_{r+1}} \ldots t_{i_{n}}\right) \in T_{n-r}$, we have

$$
\begin{aligned}
& \tilde{\Psi}_{I, \lambda}\left(\left(s_{1}, \ldots, s_{2 g}\right),\left(t_{I_{1}} h_{1}^{\left[I_{1}\right]}\left(t_{I_{1}}\right)^{-1}, \ldots, t_{I_{1}} h_{2 g}^{\left[I_{1}\right]}\left(t_{I_{1}}\right)^{-1}, 0\right)\right. \\
& \quad\left(\left(t_{I_{2}} h_{1}^{\left[I_{2}\right]}\left(t_{I_{2}}\right)^{-1}, \ldots, t_{I_{2}} h_{2 g}^{\left[I_{2}\right]}\left(t_{I_{2}}\right)^{-1}, 0\right)\right) \\
& \quad=\tilde{t} \tilde{\Psi}_{I, \lambda}\left(\left(s_{1}, \ldots, s_{2 g}\right),\left(h_{1}^{\left[I_{1}\right]}, \ldots, h_{2 g}^{\left[I_{1}\right]}, 0\right),\left(h_{1}^{\left[I_{2}\right]}, \ldots, h_{2 g}^{\left[I_{2}\right]}, 0\right)\right)
\end{aligned}
$$

for some $\tilde{t}=\left(\tilde{t}_{1}, \ldots, \tilde{t}_{n}\right)$ satisfying $\prod_{j=1}^{n} \tilde{t}_{j}=1$ and $\tilde{t}_{1}=\tilde{t}_{2}$. For any $s \in U(1)$, we may conjugate all the $h_{j}^{\left[I_{1}\right]}$ by $s^{n-r}$ and all the $h_{j}^{\left[I_{2}\right]}$ by $s^{-r}$ without changing the image under $\tilde{\Psi}_{I, \lambda}$; choosing $s$ so that $s^{n-r} \tilde{t}_{1}=s^{-r} \tilde{t}_{2}$ we find that the 
equation (7.2) is satisfied for $\tilde{t}_{j}=t_{j} s^{n-r}$ (when $j \in I_{1}$ ) and $\tilde{t}_{j}=t_{j} s^{-r}$ (when $\left.j \in I_{2}\right)$.

To show that $\Psi_{I, \lambda}$ is finite-to-one and surjective, suppose that $\left(h_{1}, \ldots, h_{2 g}, \Lambda\right) \in \tilde{F}_{I, \lambda}$; we must check that a finite (and nonzero) number of $T_{r} \times T_{n-r}$ orbits in

$$
\left(S^{1}\right)^{2 g} \times\left(\mu_{\mathrm{SU}(r)}^{-1}(0) \cap M_{r}\left(c\left(I_{1}, \lambda\right)\right)\right) \times\left(\mu_{\mathrm{SU}(n-r)}^{-1}(0) \cap M_{n-r}\left(c\left(I_{2},-\lambda\right)\right)\right)
$$

map into the $T_{n-1}$ orbit of $\left(h_{1}, \ldots, h_{2 g}, \Lambda\right)$. Now by the definition of $\tilde{F}_{I, \lambda}$ we have $\Lambda=2 \pi i \operatorname{diag}(\lambda,-\lambda, 0, \ldots, 0)$ and each $h_{j}$ is block diagonal of the form

$$
\left[\begin{array}{cc}
h_{j}^{\left[I_{1}\right]} & 0 \\
0 & h_{j}^{\left[I_{2}\right]}
\end{array}\right]
$$

with respect to the decomposition of $\{1, \ldots, n\}$ as $I_{1} \cup I_{2}$. So

$$
\tilde{\Psi}_{I, \lambda}\left(\left(s_{1}, \ldots, s_{2 g}\right),\left(H_{1}^{\left[I_{1}\right]}, \ldots, H_{2 g}^{\left[I_{1}\right]}, 0\right),\left(H_{1}^{\left[I_{2}\right]}, \ldots, H_{2 g}^{\left[I_{2}\right]}, 0\right)\right)
$$

belongs to the $T_{n-1}$ orbit of $\left(h_{1}, \ldots, h_{2 g}, \Lambda\right)$ if and only if there is some $\tilde{t}=$ $\left(\tilde{t}_{1}, \ldots, \tilde{t}_{n}\right)$ satisfying $\prod_{j=1}^{n} \tilde{t}_{j}=1$ and $\tilde{t}_{1}=\tilde{t}_{2}$ such that

$$
s_{j}^{n-r} H_{j}^{\left[I_{1}\right]}=\tilde{t}_{I_{1}} h_{j}^{\left[I_{1}\right]}\left(\tilde{t}_{I_{1}}\right)^{-1}
$$

and

$$
s_{j}^{-r} H_{j}^{\left[I_{2}\right]}=\tilde{t}_{I_{2}} h_{j}^{\left[I_{2}\right]}\left(\tilde{t}_{I_{2}}\right)^{-1}
$$

where $\tilde{t}_{I_{1}}=\left(\tilde{t}_{i_{1}}, \ldots, \tilde{t}_{i_{r}}\right) \in T_{r}$ and $\tilde{t}_{I_{2}}=\left(\tilde{t}_{i_{r+1}}, \ldots, \tilde{t}_{i_{n}}\right) \in T_{n-r}$. Since $\operatorname{det} H_{j}^{\left[I_{1}\right]}$ $=1=\operatorname{det} H_{j}^{\left[I_{2}\right]}$ and $\operatorname{det} h_{j}^{\left[I_{1}\right]} \operatorname{det} h_{j}^{\left[I_{2}\right]}=1$, by the argument of the previous paragraph this happens if and only if $\left(s_{j}\right)^{r(n-r)}=\operatorname{det} h_{j}^{\left[I_{1}\right]}$ and $s_{j}^{n-r} H_{j}^{\left[I_{1}\right]}$ is conjugate to $h_{j}^{\left[I_{1}\right]}$ and $s_{j}^{-r} H_{j}^{\left[I_{2}\right]}$ is conjugate to $h_{j}^{\left[I_{2}\right]}$. Thus $\Psi_{I, \lambda}$ is surjective and $(r(n-r))^{2 g}$-to-one.

Remark 7.4. Note that by the definition of $c_{j}^{I, \lambda}$ (see Proposition 7.3) no proper subsequence of $\left(c_{i_{1}}^{I, \lambda}, \ldots, c_{i_{r}}^{I, \lambda}\right)$ or $\left(c_{i_{r+1}}^{I, \lambda}, \ldots, c_{i_{n}}^{I, \lambda}\right)$ has product equal to 1 , because the same is true of $\left(c_{1}, \ldots, c_{n}\right)$.

Remark 7.5. It follows from Proposition 7.3 that if $\Psi_{I, \lambda}$ is orientationpreserving (and we shall see below in Remark 7.13 that $\Psi_{I, \lambda}$ takes a natural symplectic orientation on $\left(S^{1}\right)^{2 g} \times N_{r}\left(c\left(I_{1}, \lambda\right)\right) \times N_{n-r}\left(c\left(I_{2},-\lambda\right)\right)$ to the symplectic orientation induced by $\omega$ on $\left.F_{I, \lambda}\right)$ then

$$
\int_{F_{I, \lambda}} \Phi_{n-1}\left(\eta e^{\bar{\omega}}\right)=(r(n-r))^{-2 g} \int_{\left(S^{1}\right)^{2 g} \times N_{r}\left(c\left(I_{1}, \lambda\right)\right) \times N_{n-r}\left(c\left(I_{2},-\lambda\right)\right)} \Psi_{I, \lambda}^{*} \Phi_{n-1}\left(\eta e^{\bar{\omega}}\right)
$$

where both sides are elements of $H_{\hat{T}_{1}}^{*}$. To be more precise we should replace $\eta e^{\bar{\omega}}$ on each side of this equation by its restriction to $\tilde{F}_{I, \lambda}$, and as in Proposition 6.3 
and Lemma 6.7 we use the double cover $\hat{T}_{1} \times T_{n-1} \rightarrow T_{n}$ to define

$$
\Phi_{n-1}: H_{T_{n}}^{*}\left(\tilde{F}_{I, \lambda}\right) \rightarrow H_{\hat{T}_{1}}^{*}\left(F_{I, \lambda}\right) \cong H_{\hat{T}_{1}}^{*} \otimes H^{*}\left(F_{I, \lambda}\right) .
$$

Recall from the proof of the last proposition that the homomorphism

$$
\rho_{I}: S^{1} \times \mathrm{SU}(r) \times \mathrm{SU}(n-r) \rightarrow H_{I} \subset \mathrm{SU}(n)
$$

given by

$$
\rho_{I}:(s, A, B) \mapsto\left[\begin{array}{cc}
s^{n-r} A & 0 \\
0 & s^{-r} B
\end{array}\right]
$$

with respect to the decomposition of $\{1, \ldots, n\}$ as $I_{1} \cup I_{2}$ restricts to an $r(n-r)$ to-one surjective homomorphism

$$
\rho_{I}: S^{1} \times T_{r} \times T_{n-r} \rightarrow T_{n} .
$$

It is easy to check that the inclusions of $\hat{T}_{1}, T_{r}$ and $T_{n-r}$ in $T_{n}$ induce an isomorphism

$$
\hat{\rho}_{r}: \hat{T}_{1} \times T_{r} \times T_{n-r} \rightarrow T_{n}
$$

such that $\rho_{I}$ and $\hat{\rho}_{r}$ have the same restriction to $T_{r} \times T_{n-r}$. The composition of this restriction with the natural surjection from $T_{n}$ to $T_{n} / \hat{T}_{1} \cong T_{n-1} /\left(\hat{T}_{1} \cap T_{n-1}\right)$ gives an isomorphism

$$
T_{r} \times T_{n-r} \rightarrow T_{n} / \hat{T}_{1} \cong T_{n-1} /\left(\hat{T}_{1} \cap T_{n-1}\right) .
$$

Moreover the composition of $\rho_{I}$ with the inverse of $\hat{\rho}_{r}$ defines a finite (in fact $r(n-r)$-to-one) cover

$$
\nu_{I}: S^{1} \times T_{r} \times T_{n-r} \rightarrow \hat{T}_{1} \times T_{r} \times T_{n-r}
$$

which restricts to the identity on $T_{r} \times T_{n-r}$ and induces a finite cover $\nu_{I}: S^{1} \rightarrow$ $\hat{T}_{1}$ and isomorphisms on Lie algebras and equivariant cohomology.

The argument in the proof of the last proposition to show that the map $\Psi_{I, \lambda}$ is well defined on the quotient by the action of $T_{r} \times T_{n-r}$ may be rephrased as the statement that the map

$$
\begin{aligned}
\tilde{\Psi}_{I, \lambda}: & \left(S^{1}\right)^{2 g} \times\left(\mu_{\mathrm{SU}(r)}^{-1}(0) \cap M_{r}\left(c\left(I_{1}, \lambda\right)\right)\right) \\
& \times\left(\mu_{\mathrm{SU}(n-r)}^{-1}(0) \cap M_{n-r}\left(c\left(I_{2},-\lambda\right)\right)\right) \rightarrow \tilde{F}_{I, \lambda}
\end{aligned}
$$

defined in the proof of Proposition 7.3 satisfies

$$
\begin{aligned}
& \tilde{\Psi}_{I, \lambda}(\left.t\left(\left(s_{1}, \ldots, s_{2 g}\right),\left(h_{1}^{\left[I_{1}\right]}, \ldots, h_{2 g}^{\left[I_{1}\right]}, 0\right),\left(h_{1}^{\left[I_{2}\right]}, \ldots, h_{2 g}^{\left[I_{2}\right]}, 0\right)\right)\right) \\
& \quad=\rho_{I}(t) \tilde{\Psi}_{I, \lambda}\left(\left(s_{1}, \ldots, s_{2 g}\right),\left(h_{1}^{\left[I_{1}\right]}, \ldots, h_{2 g}^{\left[I_{1}\right]}, 0\right),\left(h_{1}^{\left[I_{2}\right]}, \ldots, h_{2 g}^{\left[I_{2}\right]}, 0\right)\right)
\end{aligned}
$$

for all $t \in S^{1} \times T_{r} \times T_{n-r}$, where $S^{1}$ acts trivially. Thus $\tilde{\Psi}_{I, \lambda}$ and $\rho_{I}$ induce $\tilde{\Psi}_{I, \lambda}^{*}$ from $H_{T_{n}}^{*}\left(\tilde{F}_{I, \lambda}\right)$ to

$$
\begin{aligned}
H_{S^{1}}^{*} \otimes H^{*}\left(\left(S^{1}\right)^{2 g}\right) & \otimes H_{T_{r}}^{*}\left(\mu_{\mathrm{SU}(r)}^{-1}(0) \cap M_{r}\left(c\left(I_{1}, \lambda\right)\right)\right) \\
& \otimes H_{T_{n-r}}^{*}\left(\mu_{\mathrm{SU}(n-r)}^{-1}(0) \cap M_{n-r}\left(c\left(I_{2},-\lambda\right)\right)\right)
\end{aligned}
$$


and

$$
\begin{aligned}
& \nu_{I}^{*} \int_{\left(S^{1}\right)^{2 g} \times N_{r}\left(c\left(I_{1}, \lambda\right)\right) \times N_{n-r}\left(c\left(I_{2},-\lambda\right)\right)} \Psi_{I, \lambda}^{*} \Phi_{n-1}\left(\eta e^{\bar{\omega}}\right) \\
& \quad=\int_{\left(S^{1}\right)^{2 g} \times N_{r}\left(c\left(I_{1}, \lambda\right)\right) \times N_{n-r}\left(c\left(I_{2},-\lambda\right)\right)}\left(1 \otimes \Phi_{r} \otimes \Phi_{n-r}\right) \tilde{\Psi}_{I, \lambda}^{*}\left(\eta e^{\bar{\omega}}\right),
\end{aligned}
$$

where both sides are elements of $H_{S^{1}}^{*}$. Hence by (7.3), if $Y_{1}$ is the coordinate on $\hat{\mathbf{t}}_{1}$ given by the restriction of $Y_{1}=X_{1}-X_{2}$ on $\mathbf{t}$ and $Y_{1}^{I}$ is the coordinate on the Lie algebra of $S^{1}$ obtained from $Y_{1}$ via the isomorphism on Lie algebras induced by $\nu_{I}: S^{1} \rightarrow \hat{T}_{1}$, then

$$
\begin{aligned}
\operatorname{Res}_{Y_{1}=0} & \int_{F_{I, \lambda}} \Phi_{n-1}\left(\eta e^{\bar{\omega}}\right) \\
= & (r(n-r))^{-2 g} \operatorname{Res}_{Y_{1}^{I}=0} \\
& \int_{\left(S^{1}\right)^{2 g} \times N_{r}\left(c\left(I_{1}, \lambda\right)\right) \times N_{n-r}\left(c\left(I_{2},-\lambda\right)\right)}\left(1 \otimes \Phi_{r} \otimes \Phi_{n-r}\right) \tilde{\Psi}_{I, \lambda}^{*}\left(\eta e^{\bar{\omega}}\right) .
\end{aligned}
$$

Since $S^{1}$ acts trivially, the residue operation $\operatorname{Res}_{Y_{1}^{I}=0}: H_{S^{1}}^{*} \rightarrow \mathbb{C}$ can be extended to map

$$
\begin{aligned}
H_{S^{1}}^{*} \otimes H^{*}\left(\left(S^{1}\right)^{2 g}\right) & \otimes H_{T_{r}}^{*}\left(\mu_{\mathrm{SU}(r)}^{-1}(0) \cap M_{r}\left(c\left(I_{1}, \lambda\right)\right)\right) \\
& \otimes H_{T_{n-r}}^{*}\left(\mu_{\mathrm{SU}(n-r)}^{-1}(0) \cap M_{n-r}\left(c\left(I_{2},-\lambda\right)\right)\right)
\end{aligned}
$$

to

$$
\begin{aligned}
H^{*}\left(\left(S^{1}\right)^{2 g}\right) & \otimes H_{T_{r}}^{*}\left(\mu_{\mathrm{SU}(r)}^{-1}(0) \cap M_{r}\left(c\left(I_{1}, \lambda\right)\right)\right) \\
& \otimes H_{T_{n-r}}^{*}\left(\mu_{\mathrm{SU}(n-r)}^{-1}(0) \cap M_{n-r}\left(c\left(I_{2},-\lambda\right)\right)\right)
\end{aligned}
$$

so that it commutes with $\Phi_{r}$ and $\Phi_{n-r}$ and with integration over $N_{r}\left(c\left(I_{1}, \lambda\right)\right)$ and integration over $N_{n-r}\left(c\left(I_{2},-\lambda\right)\right)$. In particular by expressing integrals over products as iterated integrals we obtain

$$
\begin{aligned}
& \operatorname{Res}_{Y_{1}=0} \int_{F_{I, \lambda}} \Phi_{n-1}\left(\eta e^{\bar{\omega}}\right) \\
& =(r(n-r))^{-2 g} \int_{N_{r}\left(c\left(I_{1}, \lambda\right)\right)} \\
& \Phi_{r}\left(\operatorname{Res}_{Y_{1}^{I}=0} \int_{N_{n-r}\left(c\left(I_{2},-\lambda\right)\right)} \Phi_{n-r}\left(\int_{\left(S^{1}\right)^{2 g}} \tilde{\Psi}_{I, \lambda}^{*}\left(\eta e^{\bar{\omega}}\right)\right)\right) \\
& =(r(n-r))^{-2 g} \int_{N_{n-r}\left(c\left(I_{2},-\lambda\right)\right)} \\
& \Phi_{n-r}\left(\operatorname{Res}_{Y_{1}^{I}=0} \int_{N_{r}\left(c\left(I_{1}, \lambda\right)\right)} \Phi_{r}\left(\int_{\left(S^{1}\right)^{2 g}} \tilde{\Psi}_{I, \lambda}^{*}\left(\eta e^{\bar{\omega}}\right)\right)\right) .
\end{aligned}
$$

This will be important when we apply induction later. 
Recall from Lemma 6.7 that $\mathcal{F}$ is the set of components of the fixed point set of the action of $\hat{T}_{1}$ on the quotient $P^{-1}(V) \cap \mu^{-1}\left(\hat{\mathbf{t}}_{1}\right) / T_{n-1}$, where $V$ is a sufficiently small $T$-invariant neighbourhood of $c$ in $K$. Every $F \in$ $\mathcal{F}$ contains a component $F_{I, \lambda}$ of the fixed point set of the action of $\hat{T}_{1}$ on $M(c) \cap \mu^{-1}\left(\hat{\mathbf{t}}_{1}\right) / T_{n-1}$, and each $F_{I, \lambda}$ is contained in a unique $F \in \mathcal{F}$ (see Proposition 7.1 for the definition of $\left.F_{I, \lambda}\right)$. For each $I$ and $\lambda$ we now need to understand the normal bundle in $P^{-1}(V) \cap \mu^{-1}\left(\hat{\mathbf{t}}_{1}\right) / T_{n-1}$ to the component $F \in \mathcal{F}$ of the fixed point set which contains $F_{I, \lambda}$. First, we observe that there is the following decomposition:

Remark 7.6. Let $I$ be a subset of $\{3, \ldots, n\}$ with $r-1$ elements where $1 \leq r \leq n-1$, let $I_{1}=I \cup\{1\}$ and let $I_{2}=\{1, \ldots, n\}-I_{1}$. Then

$$
\mathcal{D}_{n}(X)=\mathcal{D}_{r}^{\left[I_{1}\right]}(X) \mathcal{D}_{n-r}^{\left[I_{2}\right]}(X) \tau_{I}(X)
$$

where

$$
\mathcal{D}_{r}^{\left[I_{1}\right]}(X)=\prod_{1 \leq j<k \leq r}\left(X_{i_{j}}-X_{i_{k}}\right)
$$

is the product of the positive roots of $\mathrm{SU}(r)$ embedded in $\mathrm{SU}(n)$ via the inclusion of $I_{1}$ in $\{1, \ldots, n\}$,

$$
\mathcal{D}_{n-r}^{\left[I_{2}\right]}(X)=\prod_{r+1 \leq j<k \leq n}\left(X_{i_{j}}-X_{i_{k}}\right)
$$

is the product of the positive roots of $\mathrm{SU}(n-r)$ embedded in $\mathrm{SU}(n)$ via the inclusion of $I_{2}$ in $\{1, \ldots, n\}$, and

$$
\tau_{I}(X)= \pm \prod_{1 \leq j \leq r<k \leq n}\left(X_{i_{j}}-X_{i_{k}}\right)
$$

where the sign is + or - depending on whether the permutation

$$
\left[\begin{array}{cccc}
1 & 2 & \ldots & n \\
i_{1} & i_{2} & \ldots & i_{n}
\end{array}\right]
$$

is even or odd. Note also that

$$
(-1)^{r(n-r)}\left(\tau_{I}(X)\right)^{2}=\prod_{(i, j) \in I_{1} \times I_{2} \cup I_{2} \times I_{1}}\left(X_{i}-X_{j}\right) .
$$

Now we can find the $\hat{T}_{1}$-equivariant Euler class of the normal bundle in $P^{-1}(V) \cap \mu^{-1}\left(\hat{\mathbf{t}}_{1}\right) / T_{n-1}$ to the component $F \in \mathcal{F}$ of the fixed point set which contains $F_{I, \lambda}$.

LEMMA 7.7. Let $I$ be a subset of $\{3, \ldots, n\}$ with $r-1$ elements where $1 \leq r \leq n-1$, and let $\lambda \in \mathbb{R}$ be a solution of the equation

$$
e^{-2 \pi i \lambda}=\prod_{j \in I_{1}} c_{j} .
$$


Then the $\hat{T}_{1}$-equivariant Euler class of the normal bundle in $P^{-1}(V) \cap$ $\mu^{-1}\left(\hat{\mathbf{t}}_{1}\right) / T_{n-1}$ to the component $F \in \mathcal{F}$ of the fixed point set of the action of $\hat{T}_{1}$ on $P^{-1}(V) \cap \mu^{-1}\left(\hat{\mathbf{t}}_{1}\right) / T_{n-1}$ which contains $F_{I, \lambda}$ is given by $e_{F}=$ $(-1)^{r(n-r) g} \Phi_{n-1}\left(\tau_{I}^{2 g}\right)$.

Proof. The proof of Proposition 7.1 shows that the component $F \in \mathcal{F}$ of the fixed point set of the action of $\hat{T}_{1}$ on $P^{-1}(V) \cap \mu^{-1}\left(\hat{\mathbf{t}}_{1}\right) / T_{n-1}$ which contains $F_{I, \lambda}$ is

$$
F=P^{-1}(V) \cap\left(H_{I}^{2 g} \times \hat{\mathbf{t}}_{1}\right) / T_{n-1},
$$

whereas $\mu^{-1}\left(\hat{\mathbf{t}}_{1}\right)=K^{2 g} \times \hat{\mathbf{t}}_{1}$. The $T$-equivariant Chern roots of the normal bundle to $H_{I}^{2 g}$ in $K^{2 g}$ are $X_{i}-X_{j}$ for $(i, j) \in I_{1} \times I_{2} \cup I_{2} \times I_{1}$ with multiplicity $g$. The result follows by Remark 7.6.

LEMMA 7.8. Let $I$ be a subset of $\{3, \ldots, n\}$ with $r-1$ elements where $1 \leq r \leq n-1$, let $I_{1}=I \cup\{1\}$, let $I_{2}=\{1, \ldots, n\}-I_{1}$ and let $\lambda \in \mathbb{R}$ be $a$ solution of the equation

$$
e^{-2 \pi i \lambda}=\prod_{j \in I_{1}} c_{j}
$$

Let $F$ be the component of the fixed point set of the action of $\hat{T}_{1}$ on $P^{-1}(V) \cap$ $\mu^{-1}\left(\hat{\mathbf{t}}_{1}\right) / T_{n-1}$ which contains $F_{I, \lambda}$, where $F_{I, \lambda}$ is as defined in Proposition 7.1. We then have

$$
\begin{aligned}
\int_{F} \frac{\Phi_{n-1}\left(\eta e^{\bar{\omega}} \alpha\right)}{e_{F}} & =(-1)^{r(n-r)(g-1)} \int_{F_{I, \lambda}} \Phi_{n-1}\left(\frac{\eta e^{\bar{\omega}}}{\tau_{I}^{2 g-1}}\right) \\
& =(-1)^{r(n-r)(g-1)} \int_{F_{I, \lambda}} \Phi_{n-1}\left(\frac{\mathcal{D}_{r}^{\left[I_{1}\right]}(X) \mathcal{D}_{n-r}^{\left[I_{2}\right]}(X) \eta e^{\bar{\omega}}}{\mathcal{D}_{n}(X) \tau_{I}^{2 g-2}}\right)
\end{aligned}
$$

where $\alpha$ is the $T_{n}$-equivariant differential form on $K^{2 g} \times \mathbf{k}$ given by Proposition 5.7 which is supported near $M(c)$ and represents the equivariant Poincaré dual of $M(c)$ in $K^{2 g} \times \mathbf{k}$.

Proof. The $T_{n}$-equivariant differential form $\alpha$ on $K^{2 g} \times \mathbf{k}$ which represents the equivariant Poincare dual of $M(c)=P^{-1}(c)$ in $K^{2 g} \times \mathbf{k}$ was defined in Proposition 5.7 as a pullback via the map $P: K^{2 g} \times \mathbf{k} \rightarrow K$. By using the restriction $P: H_{I}^{2 g} \times \hat{\mathbf{t}}_{1} \rightarrow H_{I}$ we can similarly define a $T_{n}$-equivariant differential form $\alpha_{I}$ on $H_{I}^{2 g} \times \hat{\mathbf{t}}_{1}$ which represents the equivariant Poincaré dual of $M(c) \cap\left(H_{I}^{2 g} \times \hat{\mathbf{t}}_{1}\right)$ in $H_{I}^{2 g} \times \hat{\mathbf{t}}_{1}$. The restriction of $\Phi_{n-1}\left(\alpha_{I}\right)$ then represents the Poincaré dual to $F_{I, \lambda}$ in $F$, provided suitable orientations are chosen. Note that $\{1, \ldots, 1\} \times \mathbf{k}$ is transverse to both $M(c)=P^{-1}(c)$ and $\mu^{-1}(0)=K^{2 g} \times\{0\}$ in $K^{2 g} \times \mathbf{k}$, and that if $\Lambda \in \mathbf{k}$ then

$$
\mu(1, \ldots, 1, \Lambda)=-\Lambda
$$


while

$$
P(1, \ldots, 1, \Lambda)=\exp (-\Lambda) .
$$

From the orientation conventions of Remark 3.5 it follows that the normal to $P^{-1}\left(H_{I}\right)$ in $K^{2 g} \times \mathbf{k}$ is $T_{n}$-equivariantly isomorphic to the kernel of the restriction map $\mathbf{k}^{*} \rightarrow \mathbf{h}_{I}^{*}$. Thus the restriction of $(-1)^{r(n-r)} \tau_{I} \alpha_{I}$ to $H_{I}^{2 g} \times \hat{\mathbf{t}}_{1}$ has compact support near $M(c)$ and locally represents the equivariant Poincaré dual to $M(c)$ in $K^{2 g} \times \mathbf{k}$, so we can substitute it for $\alpha$ on $H_{I}^{2 g} \times \hat{\mathbf{t}}_{1}$ and we can substitute $(-1)^{r(n-r)} \Phi_{n-1}\left(\tau_{I} \alpha_{I}\right)$ for $\Phi_{n-1}(\alpha)$ on $F$.

We have that $e_{F}=(-1)^{r(n-r) g} \Phi_{n-1}\left(\tau_{I}^{2 g}\right)$ by the last lemma. We therefore get

$$
\begin{aligned}
\int_{F} \frac{\Phi_{n-1}\left(\eta e^{\bar{\omega}} \alpha\right)}{e_{F}} & =(-1)^{r(n-r)(g-1)} \int_{F} \Phi_{n-1}\left(\frac{\eta e^{\bar{\omega}} \alpha_{I}}{\tau_{I}^{2 g-1}}\right) \\
& =(-1)^{r(n-r)(g-1)} \int_{F_{I, \lambda}} \Phi_{n-1}\left(\frac{\eta e^{\bar{\omega}}}{\tau_{I}^{2 g-1}}\right)
\end{aligned}
$$

and Remark 7.6 completes the proof.

Remark 7.9. The condition for $F \in \mathcal{F}$ to appear in the sum in the statement of Lemma 6.7 was that

$$
-\left\|\hat{e_{1}}\right\|^{2}<\left\langle\hat{e_{1}}, \mu(F)\right\rangle<0 .
$$

Let $I$ be a subset of $\{3, \ldots, n\}$ with $r-1$ elements where $1 \leq r \leq n-1$, and let $\lambda \in \mathbb{R}$ be a solution of the equation

$$
e^{-2 \pi i \lambda}=\prod_{j \in I_{1}} c_{j}
$$

If $F \in \mathcal{F}$ is the component of the fixed point set of the action of $\hat{T}_{1}$ on $P^{-1}(V) \cap$ $\mu^{-1}\left(\hat{\mathbf{t}}_{1}\right) / T_{n-1}$ which contains $F_{I, \lambda}$, then

$$
\mu_{\hat{T}_{1}}(F)=\mu_{\hat{T}_{1}}\left(F_{I, \lambda}\right)=-\lambda \hat{e}_{1} .
$$

We thus find that for each $I$ there is precisely one solution $\lambda \in \mathbb{R}$ to the equation

$$
e^{-2 \pi i \lambda}=\prod_{j \in I_{1}} c_{j}
$$

such that the component $F$ of the fixed point set of the action of $\hat{T}_{1}$ on $P^{-1}(V) \cap$ $\mu^{-1}\left(\hat{\mathbf{t}}_{1}\right) / T_{n-1}$ which contains $F_{I, \lambda}$ contributes to the sum in Lemma 6.7. This solution is $\lambda=\delta_{I}$ where $\delta_{I}$ is the noninteger part of

$$
\frac{i}{2 \pi} \log \prod_{j \in I_{1}} c_{j}
$$

and so we have

$$
\mu_{\hat{T}_{1}}(F)=-\delta_{I} \hat{e}_{1}
$$


(Note that since $\prod_{j \in I_{1}} c_{j}$ has modulus 1 but is not equal to 1 , the noninteger part of $\frac{i}{2 \pi} \log \prod_{j \in I_{1}} c_{j}$ is well defined as an element of the open interval $(0,1)$ in $\mathbb{R}$.) We therefore define

$$
F_{I}=F_{I, \delta_{I}}
$$

and also $\Psi_{I}=\Psi_{I, \delta_{I}}$ and $\tilde{\Psi}_{I}=\tilde{\Psi}_{I, \delta_{I}}$.

We can now deduce the following result:

Proposition 7.10. If $\eta(X)$ is a polynomial in $\tilde{a}_{r}(X)$ and $\tilde{b}_{r}^{j}(X)$, so that $s_{\hat{e_{1}}}^{*} \eta=\eta$, then

$$
\begin{aligned}
\int_{N_{n}(c)} & \Phi_{n}\left(\eta e^{\bar{\omega}}\right)-\int_{N_{n}(c)} \Phi_{n}\left(\eta e^{\bar{\omega}} e^{-Y_{1}}\right)=\int_{N_{n}(c)} \Phi_{n}\left(\left(1-e^{-Y_{1}}\right) \eta e^{\bar{\omega}}\right) \\
& =\sum_{1 \leq r \leq n-1} \sum_{I \subseteq\{3, \ldots, n\},|I|=r-1}(-1)^{r(n-r)(g-1)} \operatorname{Res}_{Y_{1}=0} \int_{F_{I}} \Phi_{n-1}\left(\frac{\eta e^{\bar{\omega}}}{\tau_{I}^{2 g-1}}\right) .
\end{aligned}
$$

Proof. Recall that the coordinates $Y_{k}=e_{k}(X)=\left\langle\hat{e}_{k}, X\right\rangle$ were introduced in Definition 6.6. The result then follows immediately from Lemma 6.7, Lemma 7.8 and Remark 7.4 above, together with Lemma 6.1 and Remark 7.2.

Remark 7.11. This proposition is also true for formal equivariant cohomology classes $\eta=\sum_{j=0}^{\infty} \eta_{j}$ with $\eta_{j} \in H_{K}^{j}(M(c))$, because all but.finitely many $\eta_{j}$ contribute zero to both sides of the equations.

Corollary 7.12. Suppose $\eta$ is a polynomial in $\tilde{a}_{r}(X)$ and $\tilde{b}_{r}^{j}(X)$, so that $s_{e_{1}}^{*} \eta=\eta$. Then

$$
\begin{aligned}
\int_{N_{n}(c)} \Phi_{n}\left(\mathcal{D}_{n} \eta e^{\bar{\omega}}\right)= & \sum_{1 \leq r \leq n-1} \sum_{I \subseteq\{3, \ldots, n\},|I|=r-1}(-1)^{r(n-r)(g-1)} \operatorname{Res}_{Y_{1}=0} \\
& \int_{F_{I}} \Phi_{n-1}\left(\frac{\mathcal{D}_{r}^{\left[I_{1}\right]} \mathcal{D}_{n-r}^{\left[I_{2}\right]} \eta e^{\bar{\omega}}}{\tau_{I}^{2 g-2}\left(1-e^{-Y_{1}}\right)}\right) .
\end{aligned}
$$

Proof. This follows by application of Remark 7.6 and Proposition 7.10 with $\eta$ replaced by the formal equivariant cohomology class $\eta \mathcal{D}_{n} /\left(1-e^{-Y_{1}}\right)$. This is valid by Remark 7.11 because $Y_{1}$ divides $\mathcal{D}_{n}(X)$ and so $\mathcal{D}_{n} /\left(1-e^{-Y_{1}}\right)$ can be expressed as a power series in $Y_{1}$ whose coefficients are polynomials in the other coordinates $Y_{2}, \ldots, Y_{n-1}$.

Remark 7.13. Recall from the proof of Proposition 7.3 that

$$
\begin{aligned}
\tilde{\Psi}_{I}:\left(S^{1}\right)^{2 g} \times\left(\mu_{\mathrm{SU}(r)}^{-1}(0) \cap M_{r}\left(c\left(I_{1}, \delta_{I}\right)\right)\right) \\
\quad \times\left(\mu_{\mathrm{SU}(n-r)}^{-1}(0) \cap M_{n-r}\left(c\left(I_{2},-\delta_{I}\right)\right)\right) \rightarrow \tilde{F}_{I, \delta_{I}}
\end{aligned}
$$


is defined for $\delta_{I}$ as in Remark 7.9 by

$$
\begin{aligned}
\tilde{\Psi}_{I}\left(\left(s_{1}, \ldots, s_{2 g}\right),\left(h_{1}^{\left[I_{1}\right]}, \ldots, h_{2 g}^{\left[I_{1}\right]}, 0\right),\left(h_{1}^{\left[I_{2}\right]}, \ldots, h_{2 g}^{\left[I_{2}\right]}, 0\right)\right) \\
=\left(\left(\rho_{I}\left(s_{1}, h_{1}^{\left[I_{1}\right]}, h_{1}^{\left[I_{2}\right]}\right), \rho_{I}\left(s_{2}, h_{2}^{\left[I_{1}\right]}, h_{2}^{\left[I_{2}\right]}\right), \ldots, \rho_{I}\left(s_{2 g}, h_{2 g}^{\left[I_{1}\right]}, h_{2 g}^{\left[I_{2}\right]}\right),\right.\right. \\
\left.\quad 2 \pi i \operatorname{diag}\left(\delta_{I},-\delta_{I}, 0, \ldots, 0\right)\right)
\end{aligned}
$$

when we use the map

$$
\rho_{I}: S^{1} \times \mathrm{SU}(r) \times \mathrm{SU}(n-r) \rightarrow S(U(r) \times U(n-r)) \subset \mathrm{SU}(n)
$$

given by

$$
\rho_{I}:(s, A, B) \mapsto\left[\begin{array}{cc}
s^{n-r} A & 0 \\
0 & s^{-r} B
\end{array}\right]
$$

with respect to the decomposition of $\{1, \ldots, n\}$ as $I_{1} \cup I_{2}$, which restricts to an $r(n-r)$-to-one surjective homomorphism

$$
\rho_{I}: S^{1} \times T_{r} \times T_{n-r} \rightarrow T_{n} .
$$

Since $\bar{\omega}=\omega+\mu$ is constructed using the inner product $<,>$ defined at $(2.2)$ on the Lie algebra $\mathbf{k}$ of $K=\mathrm{SU}(n)$, and since $\rho_{I}$ embeds the Lie algebras of $S^{1}, \mathrm{SU}(r)$ and $\mathrm{SU}(n-r)$ as mutually orthogonal subspaces of $\mathbf{k}$,

$$
\tilde{\Psi}_{I}^{*}(\bar{\omega})=\bar{\omega}_{r}+\bar{\omega}_{n-r}+\Omega-\delta_{I} \hat{e}_{1}
$$

for some $\Omega \in H^{2}\left(\left(S^{1}\right)^{2 g}\right)$, where $\bar{\omega}_{r}$ and $\bar{\omega}_{n-r}$ are defined like $\bar{\omega}$ but with $n$ replaced by $r$ and $n-r$. Thus we have

$$
\tilde{\Psi}_{I}^{*}\left(\frac{\mathcal{D}_{r}^{\left[I_{1}\right]} \mathcal{D}_{n-r}^{\left[I_{2}\right]} \eta e^{\bar{\omega}}}{\tau_{I}^{2 g-2}\left(1-e^{-Y_{1}}\right)}\right)=e^{\bar{\omega}_{r}+\bar{\omega}_{n-r}+\Omega-\delta_{I} Y_{1}} \tilde{\Psi}_{I}^{*}\left(\frac{\mathcal{D}_{r}^{\left[I_{1}\right]} \mathcal{D}_{n-r}^{\left[I_{2}\right]} \eta}{\tau_{I}^{2 g-2}\left(1-e^{-Y_{1}}\right)}\right) .
$$

Since $\tilde{\Psi}_{I}^{*}\left(\mathcal{D}_{r}^{\left[I_{1}\right]}\right)=\mathcal{D}_{r}$ and $\tilde{\Psi}_{I}^{*}\left(\mathcal{D}_{n-r}^{\left[I_{2}\right]}\right)=\mathcal{D}_{n-r}$, we can combine this with Corollary 7.12 and Remark 7.5 to obtain the result on which is based the inductive proof of Witten's formulas in the next section.

Proposition 7.14. If $c \in T$ satisfies the conditions of Remark 6.4, and if $\eta(X)$ is a polynomial in $\tilde{a}_{r}(X)$ and $\tilde{b}_{r}^{j}(X)$ so that $s_{\hat{e}_{1}}^{*} \eta=\eta$, then

$$
\begin{aligned}
\int_{N_{n}(c)} \Phi_{n}\left(\mathcal{D}_{n} \eta e^{\bar{\omega}}\right)= & \sum_{1 \leq r \leq n-1} \sum_{I \subseteq\{3, \ldots, n\},|I|=r-1}(-1)^{r(n-r)(g-1)} \operatorname{Res}_{Y_{1}=0} \\
& \int_{F_{I}} \Phi_{n-1}\left(\frac{\mathcal{D}_{r}^{\left[I_{1}\right]} \mathcal{D}_{n-r}^{\left[I_{2}\right]} \eta e^{\bar{\omega}}}{\tau_{I}^{2 g-2}\left(1-e^{-Y_{1}}\right)}\right)
\end{aligned}
$$

where

$$
\operatorname{Res}_{Y_{1}=0} \int_{F_{I}} \Phi_{n-1}\left(\frac{\mathcal{D}_{r}^{\left[I_{1}\right]} \mathcal{D}_{n-r}^{\left[I_{2}\right]} \eta e^{\bar{\omega}}}{\tau_{I}^{2 g-2}\left(1-e^{-Y_{1}}\right)}\right)
$$


is equal to $(r(n-r))^{-2 g}$ times

$$
\begin{aligned}
\int_{N_{r}\left(c\left(I_{1}, \delta_{I}\right)\right)} \Phi_{r}\left(\mathcal{D}_{r} e^{\bar{\omega}_{r}} \operatorname{Res}_{Y_{1}^{I}=0} \int_{N_{n-r}\left(c\left(I_{2},-\delta_{I}\right)\right)}\right. \\
\left.\quad \times \Phi_{n-r}\left(\mathcal{D}_{n-r} e^{\bar{\omega}_{n-r}} \int_{\left(S^{1}\right)^{2 g}} e^{\Omega} \tilde{\Psi}_{I}^{*}\left(\frac{\eta e^{-\delta_{I} Y_{1}}}{\tau_{I}^{2 g-2}\left(1-e^{-Y_{1}}\right)}\right)\right)\right)
\end{aligned}
$$

and also to $(r(n-r))^{-2 g}$ times

$$
\begin{aligned}
\int_{N_{n-r}\left(c\left(I_{2},-\delta_{I}\right)\right)} \Phi_{n-r} & \left(\mathcal{D}_{n-r} e^{\bar{\omega}_{n-r}} \operatorname{Res}_{Y_{1}^{I}=0} \int_{N_{r}\left(c\left(I_{1}, \delta_{I}\right)\right)}\right. \\
& \left.\times \Phi_{r}\left(\mathcal{D}_{r} e^{\bar{\omega}_{r}} \int_{\left(S^{1}\right)^{2 g}} e^{\Omega} \tilde{\Psi}_{I}^{*}\left(\frac{\eta e^{-\delta_{I} Y_{1}}}{\tau_{I}^{2 g-2}\left(1-e^{-Y_{1}}\right)}\right)\right)\right) .
\end{aligned}
$$

Here $\left.c\left(I_{1}, \delta_{I}\right)\right)$ and $c\left(I_{2},-\delta_{I}\right)$ are defined as in Proposition 7.3 with $\delta_{I}$ as in Remark 7.4 and $\bar{\omega}_{r}, \bar{\omega}_{n-r}$ and $\Omega$ as in Remark 7.13.

Remark 7.15. For any $\gamma \in T_{n}$ a unique $\tilde{\gamma} \in \mathbf{t}_{n}$ can be chosen so that $\exp \tilde{\gamma}=\gamma$ and $\tilde{\gamma}$ belongs to the fundamental domain defined by the simple roots for the translation action on $\mathbf{t}_{n}$ of the integer lattice $\Lambda^{I}$ (i.e. $\tilde{\gamma}=\gamma_{1} \hat{e}_{1}+$ $\ldots+\gamma_{n-1} \hat{e}_{n-1}$ with $0 \leq \gamma_{j}<1$ for $\left.1 \leq j \leq n-1\right)$. Suppose that $\tilde{c}\left(I_{1}, \delta_{I}\right) \in \mathbf{t}_{r}$ and $\tilde{c}\left(I_{2},-\delta_{I}\right) \in \mathbf{t}_{n-r}$ are chosen in this way in the fundamental domains defined by the simple roots for the translation actions on $\mathbf{t}_{r}$ and $\mathbf{t}_{n-r}$ of their integer lattices, satisfying

$$
\exp \tilde{c}\left(I_{1}, \delta_{I}\right)=c\left(I_{1}, \delta_{I}\right)=\operatorname{diag}\left(c_{i_{1}}^{I, \delta_{I}}, \ldots, c_{i_{r}}^{I, \delta_{I}}\right)
$$

and

$$
\exp \tilde{c}\left(I_{2},-\delta_{I}\right)=c\left(I_{2},-\delta_{I}\right)=\operatorname{diag}\left(c_{i_{r+1}}^{I, \delta_{I}}, \ldots, c_{i_{n}}^{I, \delta_{I}}\right),
$$

where (as in Proposition 7.3 and Remark 7.9) we define $\delta_{I}$ to be the noninteger part of $\frac{i}{2 \pi} \log \prod_{j \in I_{1}} c_{j}$ and let $c_{j}^{I, \delta_{I}}=c_{j}$ if $j \geq 3$, and $c_{1}^{I, \delta_{I}}=c_{1} e^{2 \pi i \delta_{I}}$ and $c_{2}^{I, \delta_{I}}=c_{2} e^{-2 \pi i \delta_{I}}$.

In the proof of the main theorem (Theorem 8.1) of the next section we shall need to consider the elements $w_{I}^{1}$ and $w_{I}^{2}$ of the subgroup $S_{n-1}$ of the Weyl group $W \cong S_{n}$ of $\mathrm{SU}(n)$ given by the permutations

$$
\left[\begin{array}{cccc}
1 & 2 & \ldots & n \\
i_{1} & i_{2} & \ldots & i_{n}
\end{array}\right]
$$

and

$$
\left[\begin{array}{lcccccc}
1 & 2 & \ldots & n-r & n-r+1 & \ldots & n \\
i_{r+1} & i_{r+2} & \ldots & i_{n} & i_{1} & \ldots & i_{r}
\end{array}\right]
$$


in the cases when $i_{r}=1$ and $i_{r+1}=2$ and $i_{n}=n$ and when $i_{1}=1$ and $i_{n}=2$ and $i_{r}=n$ respectively. We will use the fact that if $i_{r}=1$ and $i_{r+1}=2$ and $i_{n}=n$ then

$$
w_{I}^{1}(\tilde{c})=\left[\begin{array}{cc}
\tilde{c}\left(I_{1}, \delta_{I}\right) & 0 \\
0 & \tilde{c}\left(I_{2},-\delta_{I}\right)
\end{array}\right]+\left(1-\delta_{I}\right) \hat{e}_{1},
$$

where the block diagonal form is taken with respect to the decomposition of $\{1, \ldots, n\}$ as $\{1, \ldots, r\} \cup\{r+1, \ldots, n\}$. To see why this is the case, note that

$$
w_{I}^{1}(\tilde{c})(X)=\gamma_{1}\left(X_{i_{1}}-X_{i_{2}}\right)+\ldots \gamma_{n-1}\left(X_{i_{n-1}}-X_{i_{n}}\right)
$$

where $\gamma_{k}$ is the noninteger part of $\frac{1}{2 \pi i} \log \prod_{j \leq k} c_{i_{j}}$, so that $\gamma_{r}=1-\delta_{I}$ and if $k<r$ then $\gamma_{k}$ is the noninteger part of $\frac{1}{2 \pi i} \log \prod_{j \leq k} c_{i_{j}}^{I, \delta_{I}}$ whereas if $k>r$ then $\gamma_{k}$ is the noninteger part of

$$
-\delta_{I}+\frac{1}{2 \pi i} \log \prod_{r<j \leq k} c_{i_{j}}=\frac{1}{2 \pi i} \log \prod_{r<j \leq k} c_{i_{j}}^{I, \delta_{I}} .
$$

Similarly if $i_{1}=1$ and $i_{n}=2$ and $i_{r}=n$ then

$$
w_{I}^{2}(\tilde{c})=\left[\begin{array}{cc}
\tilde{c}\left(I_{2},-\delta_{I}\right) & 0 \\
0 & \tilde{c}\left(I_{1}, \delta_{I}\right)
\end{array}\right]-\delta_{I} \hat{e}_{1}
$$

where the block diagonal form is taken with respect to the decomposition of $\{1, \ldots, n\}$ as $\{1, \ldots, n-r\} \cup\{n-r+1, \ldots, n\}$.

\section{Proof of the iterated residue formula}

In this section we shall use induction to prove Witten's formulas as given in Section 2 (see Proposition 2.2) involving iterated residues, for pairings of the form

$$
\prod_{r=2}^{n} a_{r}^{m_{r}} \prod_{k_{r}=1}^{2 g}\left(b_{r}^{k_{r}}\right)^{p_{r, k_{r}}} \exp \left(f_{2}\right)[\mathcal{M}(n, d)]
$$

for nonnegative integers $m_{r}$ and $p_{r, k}$. The induction is based on Proposition 7.14. In the next section we shall extend the proof to give formulas for all pairings, and in the following section we shall show that these formulas are equivalent to those of Witten.

We are aiming to prove the following:

TheOREM 8.1. Let $c=\operatorname{diag}\left(e^{2 \pi \mathrm{id} / n}, \ldots, e^{2 \pi \mathrm{id} / n}\right)$ where $d \in$ $\{1, \ldots, n-1\}$ is coprime to $n$, and suppose that $\eta \in H_{\mathrm{SU}(n)}^{*}\left(M_{n}(c)\right)$ is a polynomial $Q\left(\tilde{a}_{2}, \ldots, \tilde{a}_{n}, \tilde{b}_{2}^{1}, \ldots, \tilde{b}_{n}^{2 g}\right)$ in the equivariant cohomology classes $\tilde{a}_{r}$ and $\tilde{b}_{r}^{j}$ 
for $2 \leq r \leq n$ and $1 \leq j \leq 2 g$. Then the pairing $Q\left(a_{2}, \ldots, a_{n}, b_{2}^{1}, \ldots, b_{n}^{2 g}\right)$ $\exp \left(f_{2}\right)[\mathcal{M}(n, d)]$ is given by

$$
\begin{aligned}
\int_{\mathcal{M}(n, d)} \Phi\left(\eta e^{\bar{\omega}}\right)= & \frac{(-1)^{n_{+}(g-1)}}{n !} \operatorname{Res}_{Y_{1}=0} \ldots \operatorname{Res}_{Y_{n-1}=0} \\
& \times\left(\frac{\sum_{w \in W_{n-1}} e^{\langle[[w \tilde{c}]], X\rangle} \int_{T_{n}{ }^{2 g}} \eta e^{\omega}}{\mathcal{D}_{n}^{2 g-2} \prod_{1 \leq j \leq n-1}\left(\exp \left(Y_{j}\right)-1\right)}\right),
\end{aligned}
$$

where $n_{+}=\frac{1}{2} n(n-1)$ is the number of positive roots of $K=\mathrm{SU}(n)$ and $X \in T_{n}$ has coordinates $Y_{1}=X_{1}-X_{2}, \ldots, Y_{n-1}=X_{n-1}-X_{n}$ defined by the simple roots, while $W_{n-1} \cong S_{n-1}$ is the Weyl group of $\mathrm{SU}(n-1)$ embedded in $\mathrm{SU}(n)$ in the standard way using the first $n-1$ coordinates. The element $\tilde{c}$ was defined in Remark 2.3: it is the unique element of $\mathbf{t}_{n}$ which satisfies $e^{2 \pi i \tilde{c}}=c$ and belongs to the fundamental domain defined by the simple roots for the translation action on $\mathbf{t}_{n}$ of the integer lattice $\Lambda^{I}$. Also, the notation $[[\gamma]]$ (introduced in Definition 2.1) means the unique element which is in the fundamental domain defined by the simple roots for the translation action on $\mathbf{t}_{n}$ of the integer lattice and for which $[[\gamma]]$ is equal to $\gamma$ plus some element of the integer lattice.

Remark 8.2. Here the integral

$$
\int_{T_{n}^{2 g}} \eta e^{\omega}
$$

is to be interpreted as the integral of the restriction of $\eta e^{\omega}$ over a connected component

$$
T_{n}^{2 g} \times\{\lambda\}
$$

(for some $\lambda \in \mathbf{t}_{n}$ satisfying $c \exp \lambda=1$ ) of the fixed point set of the action of $T_{n}$ on $M_{n}(c)$. It does not matter which component we choose here, because $\eta$ and $\omega$ are invariant under the translation maps $s_{\Lambda_{0}}$ defined in Lemma 4.5 for $\Lambda_{0}$ in the integer lattice of $\mathbf{t}_{n}$.

Remark 8.3(a). We can substitute $-X$ for $X$ in Theorem 8.1 to get

$$
\begin{aligned}
\int_{\mathcal{M}(n, d)} \Phi\left(\eta e^{\bar{\omega}}\right)= & \frac{(-1)^{n_{+}(g-1)}}{n !} \operatorname{Res}_{Y_{1}=0} \ldots \operatorname{Res}_{Y_{n-1}=0} \\
& \left(\frac{\sum_{w \in W_{n-1}} e^{-\langle[[w \tilde{c}], X\rangle} \int_{T_{n}{ }^{2 g}} \eta(-X) e^{\omega}}{\mathcal{D}_{n}^{2 g-2} \prod_{1 \leq j \leq n-1}\left(1-\exp \left(-Y_{j}\right)\right)}\right) .
\end{aligned}
$$

(b) When $\eta$ is a polynomial in $a_{2}, \ldots, a_{n}$ then

$$
\int_{T_{n}^{2 g}} \eta e^{\omega}=\eta \int_{T_{n}^{2 g}} e^{\omega}=n^{g} \eta
$$

(see Lemma 10.7 below). Since $\tilde{a}_{r}$ is represented by the polynomial $\tau_{r}(-X)$ for $2 \leq r \leq n$ (see Proposition 4.4 or Section 9 below), this means that, by (a) 
above, Theorem 8.1 combined with Proposition 2.2 gives us Witten's formula (2.4).

(c) We can also replace the symplectic form $\omega$ by any nonzero scalar multiple $\varepsilon \omega$. Then the moment map $\mu$ is multiplied by the same scalar $\varepsilon$, and the proof of Theorem 8.1 yields

$$
\begin{aligned}
\int_{\mathcal{M}(n, d)} \Phi\left(\eta e^{\varepsilon \bar{\omega}}\right)= & \frac{(-1)^{n_{+}(g-1)}}{n !} \operatorname{Res}_{Y_{1}=0} \ldots \operatorname{Res}_{Y_{n-1}=0} \\
& \left(\frac{\sum_{w \in W_{n-1}} e^{\langle\varepsilon[[w \tilde{c}]], X\rangle} \int_{T_{n} 2 g} \eta e^{\varepsilon \omega}}{\mathcal{D}_{n}^{2 g-2} \prod_{1 \leq j \leq n-1}\left(\exp \left(\varepsilon Y_{j}\right)-1\right)}\right) .
\end{aligned}
$$

If the degree of $\eta$ is equal to the dimension of $\mathcal{M}(n, d)$ then the left-hand side of this equation is equal to

$$
\int_{\mathcal{M}(n, d)} \Phi(\eta)
$$

and hence is independent of $\varepsilon$. Thus in this case we can take any nonzero value of $\varepsilon$ on the right-hand side, or let $\varepsilon$ tend to zero, to give alternative formulas for $\int_{\mathcal{M}(n, d)} \Phi(\eta)$.

Recall from Lemma 6.1 that

$$
\int_{\mathcal{M}(n, d)} \Phi\left(\eta e^{\bar{\omega}}\right)=\frac{1}{n !} \int_{N_{n}(c)} \Phi_{n}\left(\mathcal{D}_{n} \eta e^{\bar{\omega}}\right) .
$$

Proposition 7.14 tells us that $\int_{N_{n}(c)} \Phi_{n}\left(\mathcal{D}_{n} \eta e^{\bar{\omega}}\right)$ can be expressed in terms of iterated integrals of the same form for smaller values of $n$, but with $c$ no longer central in $K=\mathrm{SU}(n)$. We shall therefore obtain Theorem 8.1 from the following result involving values of $c$ which are not central (cf. Remark 6.4), which will be proved by induction on $n$.

Proposition 8.4. Let $c=\operatorname{diag}\left(c_{1}, \ldots, c_{n}\right) \in T_{n}$ be such that the product of no proper subset of $c_{1}, \ldots, c_{n}$ is 1 . If $\eta(X)$ is a polynomial in the $\tilde{a}_{r}(X)$ and $\tilde{b}_{r}^{j}(X)$, so that $s_{\hat{e}_{l}}^{*} \eta=\eta$, then

$$
\begin{aligned}
\int_{N_{n}(c)} \Phi_{n}\left(\mathcal{D}_{n} \eta e^{\bar{\omega}}\right)= & (-1)^{n_{+}(g-1)} \operatorname{Res}_{1}=0 \ldots \operatorname{Res}_{Y_{n-1}}=0 \\
& \left(\frac{\sum_{w \in W_{n-1}} e^{\langle[[w \tilde{c}]], X\rangle} \int_{T_{n} 2 g} \eta e^{\omega}}{\mathcal{D}_{n}^{2 g-2} \prod_{1 \leq j \leq n-1}\left(\exp \left(Y_{j}\right)-1\right)}\right)
\end{aligned}
$$

where $W_{n-1} \cong S_{n-1}$ is the Weyl group of $\mathrm{SU}(n-1)$, embedded in $\mathrm{SU}(n)$ in the standard way using the first $n-1$ coordinates, and $\tilde{c}=\left(\tilde{c}_{1}, \ldots, \tilde{c}_{n}\right) \in \mathbf{t}_{n}$ satisfies $e^{2 \pi i \tilde{c}}=c$ and belongs to the fundamental domain defined by the simple roots for the translation action on $\mathbf{t}_{n}$ of the integer lattice $\Lambda^{I}$.

Proof of Theorem 8.1 from Proposition 8.4. Note that when $c=$ $\operatorname{diag}\left(e^{2 \pi \mathrm{id} / n}, \ldots, e^{2 \pi \mathrm{id} / n}\right)$ we had introduced an element $\tilde{c} \in \mathbf{t}_{n}$ (see Remark 2.3) 
which satisfies $e^{2 \pi i \tilde{c}}=c$ and belongs to the fundamental domain defined by the simple roots for the translation action on $T_{n}$ of the integer lattice $\Lambda^{I}$. Thus Theorem 8.1 follows immediately from (8.2) and Proposition 8.4.

Proof of Proposition 8.4. The proof is by induction on $n$. When $n=1$ then both SU(n) and the torus $T_{n}$ are trivial, $\mathcal{D}_{n}=1$ and both $M_{n}(c)$ and $N_{n}(c)$ are single points. Thus in this case Proposition 8.4 reduces to the tautology $\eta=\eta$ for any $\eta \in H_{\mathrm{SU}(1)}^{*}\left(M_{1}(c)\right)$.

Now let us assume that $n>1$ and that the result is true for all smaller values of $n$. By Proposition 7.14,

$$
\begin{aligned}
\int_{N_{n}(c)} \Phi_{n}\left(\mathcal{D}_{n} \eta e^{\bar{\omega}}\right)= & \sum_{1 \leq r \leq n-1} \sum_{I \subseteq\{3, \ldots, n\},|I|=r-1}(-1)^{r(n-r)(g-1)} \operatorname{Res}_{Y_{1}=0} \\
& \int_{F_{I}} \Phi_{n-1}\left(\frac{\mathcal{D}_{r}^{\left[I_{1}\right]} \mathcal{D}_{n-r}^{\left[I_{2}\right]} \eta e^{\bar{\omega}}}{\tau_{I}^{2 g-2}\left(1-e^{-Y_{1}}\right)}\right)
\end{aligned}
$$

where

$$
\operatorname{Res}_{Y_{1}=0} \int_{F_{I}} \Phi_{n-1}\left(\frac{\mathcal{D}_{r}^{\left[I_{1}\right]} \mathcal{D}_{n-r}^{\left[I_{2}\right]} \eta e^{\bar{\omega}}}{\tau_{I}^{2 g-2}\left(1-e^{-Y_{1}}\right)}\right)
$$

is equal to $(r(n-r))^{-2 g}$ times the iterated integral

$$
\begin{aligned}
\int_{N_{r}\left(c\left(I_{1}, \delta_{I}\right)\right)} \Phi_{r}( & \mathcal{D}_{r} e^{\bar{\omega}_{r}} \operatorname{Res}_{Y_{1}^{I}=0} \int_{N_{n-r}\left(c\left(I_{2},-\delta_{I}\right)\right)} \\
& \left.\times \Phi_{n-r}\left(\mathcal{D}_{n-r} e^{\bar{\omega}_{n-r}} \int_{\left(S^{1}\right)^{2 g}} e^{\Omega} \tilde{\Psi}_{I}^{*}\left(\frac{\eta e^{-\delta_{I} Y_{1}}}{\tau_{I}^{2 g-2}\left(1-e^{-Y_{1}}\right)}\right)\right)\right)
\end{aligned}
$$

and also to $(r(n-r))^{-2 g}$ times the iterated integral

$$
\begin{aligned}
\int_{N_{n-r}\left(c\left(I_{2},-\delta_{I}\right)\right)} \Phi_{n-r} & \left(\mathcal{D}_{n-r} e^{\bar{\omega}_{n-r}} \operatorname{Res}_{Y_{1}^{I}=0} \int_{N_{r}\left(c\left(I_{1}, \delta_{I}\right)\right)}\right. \\
& \left.\times \Phi_{r}\left(\mathcal{D}_{r} e^{\bar{\omega}_{r}} \int_{\left(S^{1}\right)^{2 g}} e^{\Omega} \tilde{\Psi}_{I}^{*}\left(\frac{\eta e^{-\delta_{I} Y_{1}}}{\tau_{I}^{2 g-2}\left(1-e^{-Y_{1}}\right)}\right)\right)\right),
\end{aligned}
$$

for $c\left(I_{1}, \delta_{I}\right)$ and $c\left(I_{2},-\delta_{I}\right)$ defined as in Proposition 7.3 with $\delta_{I}$ as in Remark 7.4 and $\tau_{I}$ as in Remark 7.6. Here $\Omega \in H^{2}\left(\left(S^{1}\right)^{2 g}\right)$ satisfies

$$
\tilde{\Psi}_{I}^{*}(\bar{\omega})=\bar{\omega}_{r}+\bar{\omega}_{n-r}+\Omega-\delta_{I} \hat{e}_{1}
$$

as in Remark 7.13.

We need to consider separately those $I$ containing $n$ and those for which $n$ is not an element of $I$; first let us suppose that $n$ is not an element of $I$. Note that

$$
(-1)^{\left(r(n-r)+\frac{1}{2} r(r-1)+\frac{1}{2}(n-r)(n-r-1)\right)(g-1)}=(-1)^{\frac{1}{2} n(n-1)(g-1)},
$$


and

$$
\frac{e^{-\delta_{I} Y_{1}}}{1-e^{-Y_{1}}}=\frac{e^{\left(1-\delta_{I}\right) Y_{1}}}{e^{Y_{1}}-1} .
$$

The finite cover $\rho_{I}: S^{1} \times T_{r} \times T_{n-r} \rightarrow T_{n}$ is $r(n-r)$-to-one, so that it induces an $(r(n-r))^{2 g}$-to-one surjection from $\left(S^{1}\right)^{2 g} \times T_{r}^{2 g} \times T_{n-r}^{2 g}$ to $T_{n}^{2 g}$ and we have

$$
\int_{T_{n}^{2 g}} \eta e^{\omega}=\int_{\left(S^{1}\right)^{2 g} \times T_{r}^{2 g} \times T_{n-r}^{2 g}} \eta e^{\omega_{r}+\omega_{n-r}+\Omega} .
$$

Moreover this finite cover $\rho_{I}: S^{1} \times T_{r} \times T_{n-r} \rightarrow T_{n}$ takes the coordinate $Y_{1}=X_{1}-X_{2}$ on $\mathbf{t}$ to the coordinate $Y_{1}^{I}$ on the Lie algebra of $S^{1}$. Since $\tilde{\Psi}_{I}^{*}$ was defined using $\rho_{I}$ (see Remark 7.5), we deduce using Remark 7.13 and Remark 7.6 and induction on $n$ that $(-1)^{r(n-r)(g-1)}$ times the iterated integral

$$
\begin{aligned}
\int_{N_{r}\left(c\left(I_{1}, \delta_{I}\right)\right)} \Phi_{r}( & \mathcal{D}_{r} e^{\bar{\omega}_{r}} \operatorname{Res}_{Y_{1}^{I}=0} \int_{N_{n-r}\left(c\left(I_{2},-\delta_{I}\right)\right)} \\
& \left.\times \Phi_{n-r}\left(\mathcal{D}_{n-r} e^{\bar{\omega}_{n-r}} \int_{\left(S^{1}\right)^{2 g}} e^{\Omega} \tilde{\Psi}_{I}^{*}\left(\frac{\eta e^{-\delta_{I} Y_{1}}}{\tau_{I}^{2 g-2}\left(1-e^{-Y_{1}}\right)}\right)\right)\right)
\end{aligned}
$$

equals $(-1)^{n_{+}(g-1)}(r(n-r))^{2 g}$ times the iterated residue

$$
\begin{gathered}
\left.\operatorname{Res}_{X_{i_{1}}}-X_{i_{2}}=0 \ldots \operatorname{Res}_{X_{i_{r-1}}-X_{i_{r}}=0} \operatorname{Res}_{X_{1}-X_{2}=0} \operatorname{Res}_{X_{i_{r+1}}-X_{i_{r+2}}=0} \ldots \operatorname{Res}_{X_{i_{1}}-X_{i_{2}}=0} \sum_{w_{1} \in W_{r-1}} \sum_{w_{2} \in W_{n-r-1}} \frac{e^{\left\langle\left[\left[w_{1} \tilde{c}\left(I_{1}, \delta_{I}\right)\right]\right], Y_{I_{1}}\right\rangle} e^{\left\langle\left[\left[w_{2} \tilde{c}\left(I_{2},-\delta_{I}\right)\right]\right], Y_{I_{2}}\right\rangle} e^{\left(1-\delta_{I}\right) Y_{1}} \int_{T_{n}^{2 g}} \eta e^{\omega}}{\mathcal{D}_{n}^{2 g-2}\left(e^{Y_{1}}-1\right) \prod_{j \neq r}\left(\exp \left(X_{i_{j}}-X_{i_{j+1}}\right)-1\right)}\right)
\end{gathered}
$$

where $Y_{I_{1}}$ and $Y_{I_{2}}$ are the projections of $X$ onto the Lie algebras of the maximal tori $T_{r}$ and $T_{n-r}$ of $\mathrm{SU}(r)$ and $\mathrm{SU}(n-r)$ embedded in $\mathrm{SU}(n)$ via the decomposition of $\{1, \ldots, n\}$ as $I_{1} \cup I_{2}$, and $W_{r-1}$ and $W_{n-r-1}$ are the Weyl groups of $\mathrm{SU}(r-1)$ and $\mathrm{SU}(n-r-1)$ embedded in $\mathrm{SU}(r)$ and $\mathrm{SU}(n-r)$ using all but the last coordinates.

There is no need to assume that $i_{1}<i_{2}<\cdots<i_{r}$ and $i_{r+1}<i_{r+2}$ $<\cdots<i_{n}$ here. We simply need that $I_{1}=I \cup\{1\}=\left\{i_{1}, \ldots, i_{r}\right\}$ and $I_{2}=$ $\{1, \ldots, n\}-I_{1}=\left\{i_{r+1}, \ldots, i_{n}\right\}$. So let us assume that

$$
i_{r}=1
$$

and

$$
i_{r+1}=2 \text {. }
$$

We are also supposing that $n$ is not an element of $I$ (i.e. that $n \in I_{2}$ ) so we may assume in addition that $i_{n}=n$. Then we can apply the Weyl transformation $w_{I}^{1} \in W_{n-1}$ given by the permutation

$$
\left[\begin{array}{ccccc}
1 & \ldots & r & \ldots & n-1 \\
i_{1} & \ldots & i_{r} & \ldots & i_{n-1}
\end{array}\right]
$$


together with Remark 7.15 to identify the iterated residue above with

$$
\begin{aligned}
& (-1)^{n_{+}(g-1)}(r(n-r))^{2 g} \operatorname{Res}_{Y_{1}=0} \ldots \operatorname{Res}_{Y_{n-1}=0} \\
& \quad \times \sum_{w_{1} \in W_{r-1}} \sum_{w_{2} \in W_{n-r-1}} \frac{e^{\left\langle\left[\left[w_{I}^{1} w_{1} w_{2}(\tilde{c})\right]\right], X\right\rangle} \int_{T_{n}^{2 g}} \eta e^{\omega}}{\mathcal{D}_{n}^{2 g-2} \prod_{1 \leq j \leq n-1}\left(1-\exp \left(-Y_{j}\right)\right)} .
\end{aligned}
$$

When $n \in I$ the argument is similar but we apply induction to $(-1)^{r(n-r)(g-1)}$ times

$$
\begin{aligned}
\int_{N_{n-r}\left(c\left(I_{2},-\delta_{I}\right)\right)} \Phi_{n-r}( & \mathcal{D}_{n-r} e^{\bar{\omega}_{n-r}} \operatorname{Res}_{Y_{1}^{I}=0} \int_{N_{r}\left(c\left(I_{1}, \delta_{I}\right)\right)} \\
& \left.\times \Phi_{r}\left(\mathcal{D}_{r} e^{\bar{\omega}_{r}} \int_{\left(S^{1}\right)^{2 g}} e^{\Omega} \tilde{\Psi}_{I}^{*}\left(\frac{\eta e^{-\delta_{I} Y_{1}}}{\tau_{I}^{2 g-2}\left(1-e^{-Y_{1}}\right)}\right)\right)\right)
\end{aligned}
$$

and observe that

$$
\operatorname{Res}_{X_{1}-X_{2}=0} \frac{e^{-\delta_{I}\left(X_{1}-X_{2}\right)}}{1-e^{-\left(X_{1}-X_{2}\right)}}=-\operatorname{Res}_{X_{2}-X_{1}=0} \frac{e^{\delta_{I}\left(X_{2}-X_{1}\right)}}{1-e^{X_{2}-X_{1}}}
$$

(see the Remark after Corollary 3.2). As $I_{1}=I \cup\{1\}=\left\{i_{1}, \ldots, i_{r}\right\}$ and $I_{2}=\{1, \ldots, n\}-I_{1}=\left\{i_{r+1}, \ldots, i_{n}\right\}$ and $n \in I$ we can assume that $i_{1}=1$, $i_{r}=n$ and $i_{n}=2$. Then we use the Weyl transformation $w_{I}^{2} \in W_{n-1}$ given by the permutation

$$
\left[\begin{array}{cccccc}
1 & \ldots & n-r & n-r+1 & \ldots & n-1 \\
i_{r+1} & \ldots & i_{n} & i_{1} & \ldots & i_{r-1}
\end{array}\right]
$$

together with Remark 7.15 to equate the iterated integral above with

$$
\operatorname{Res}_{Y_{1}=0} \ldots \operatorname{Res}_{Y_{n-1}=0} \sum_{w_{1} \in W_{r-1}} \sum_{w_{2} \in W_{n-r-1}} \frac{e^{\left\langle\left[\left[w_{I}^{2} w_{1} w_{2}(\tilde{c})\right]\right], X\right\rangle} \int_{T_{n}^{2 g}} \eta e^{\omega}}{\mathcal{D}_{n}^{2 g-2} \prod_{1 \leq j \leq n-1}\left(\exp \left(Y_{j}\right)-1\right)} .
$$

Thus it suffices to prove:

Lemma 8.5. For each subset $I$ of $\{3, \ldots, n\}$ with $r-1$ elements, fix $i_{1}, \ldots, i_{n}$ such that $I \cup\{1\}=\left\{i_{1}, \ldots, i_{r}\right\}$ and $\{2, \ldots, n\}-I=\left\{i_{r+1}, \ldots, i_{n}\right\}$ and also $i_{r}=1, i_{r+1}=2$ and $i_{n}=n($ if $n \notin I)$ or $i_{1}=1, i_{r}=n$ and $i_{n}=2$ (if $n \in I$ ). Define permutations $w_{I}^{1}$ (for I such that $n \notin I$ ) and $w_{I}^{2}$ (for I such that $n \in I)$ as above. Then as

(i) $r$ runs over $\{1, \ldots, n-1\}$,

(ii) $w_{1}$ runs over permutations of $\{1, \ldots, r\}$ fixing $r$,

(iii) $w_{2}$ runs over permutations of $\{r+1, \ldots, n\}$ fixing $n$ and

(iv) I runs over subsets of $\{3, \ldots, n\}$ with $r-1$ elements not containing $n$,

the product $w_{I}^{1} w_{1} w_{2}$ runs over the set of permutations $w$ of $\{1, \ldots, n\}$ fixing $n$ such that

$$
w^{-1}(1)<w^{-1}(2)
$$


Moreover if instead of (iv) I runs over subsets of $\{3, \ldots, n\}$ with $r-1$ elements containing $n$, then the product $w_{I}^{2} w_{1} w_{2}$ runs over the set of permutations $w$ of $\{1, \ldots, n\}$ fixing $n$ such that

$$
w^{-1}(1)>w^{-1}(2) \text {. }
$$

Proof. If $w \in W_{n-1}$ satisfies $w^{-1}(1)<w^{-1}(2)$ let $r=w^{-1}(1)$ and $I=$ $\left\{j: w^{-1}(j)<r\right\}$. On the other hand if $w \in W_{n-1}$ satisfies $w^{-1}(1)>w^{-1}(2)$ let $r=n-w^{-1}(2)$ and $I=\left\{j>1: w^{-1}(j)>n-r\right\} \cup\{n\}$. In each case it is easy to check that there exist unique choices of $w_{1}$ and $w_{2}$ such that $w_{I}^{1} w_{1} w_{2}=w$ or $w_{I}^{2} w_{1} w_{2}=w$.

This completes the proof of the lemma and hence of Proposition 8.4.

Remark 8.6. It is shown in Proposition 3.4 of [33] that the multivariable residue (multiplied by the constant $C_{K}$ ) of Theorem 3.1 and formula (6.1) can be replaced by the iterated one-variable residue

$$
\operatorname{Res}_{Y_{1}=0}^{+} \ldots \operatorname{Res}_{Y_{n-1}=0}^{+}
$$

multiplied by the Jacobian (in this case $1 / n$ ) of the change of coordinates from an orthonormal system to $\left(Y_{1}, \ldots, Y_{n-1}\right)$. Here, if $\operatorname{Res}_{y=0} g(y)$ denotes the coefficient of $y^{-1}$ in the Laurent expansion about 0 of a meromorphic function $g(y)$ of one complex variable $y$, then $\operatorname{Res}^{+}$is defined for meromorphic functions of the special form $\sum_{1 \leq i \leq s} e^{\lambda_{i} y} q_{i}(y)$, where $\lambda_{1}, \ldots, \lambda_{s}$ are real numbers and $q_{1}, \ldots, q_{s}$ are rational functions of one variable with complex coefficients, by

$$
\operatorname{Res}_{y=0}^{+}\left(\sum_{1 \leq i \leq s} e^{\lambda_{i} y} q_{i}(y)\right)=\sum_{1 \leq i \leq s, \lambda_{i}>0} \operatorname{Res}_{y=0}\left(e^{\lambda_{i} y} q_{i}(y)\right) .
$$

Since

$$
\frac{e^{\gamma y}}{e^{y}-1}
$$

can be formally expanded as

$$
-\sum_{m \in \mathbb{Z}, m+\gamma>0} e^{(m+\gamma) y}
$$

when $0<\gamma<1$, the formula (6.1) can be formally rewritten as

$$
\begin{aligned}
\prod_{r=2}^{n} a_{r}^{m_{r}} & \exp \left(f_{2}\right)[\mathcal{M}(n, d)] \\
& =\frac{(-1)^{n_{+}(g-1)}}{n} \operatorname{Res}_{Y_{1}=0}^{+} \ldots \operatorname{Res}_{Y_{n-1}=0}^{+} \frac{e^{\langle\tilde{c}, X\rangle} \int_{T_{n}^{2 g}} e^{\omega} \prod_{r=2}^{n} \tau_{r}^{m_{r}}}{\mathcal{D}_{n}^{2 g-2} \prod_{1 \leq j \leq n-1}\left(e^{Y_{j}}-1\right)} .
\end{aligned}
$$

Moreover the multivariable residue Res is invariant under the action of the Weyl group, as are all the other ingredients of the right-hand side of (6.1) 
except for $\tilde{c}$. Thus by averaging (6.1) over the Weyl group we obtain a special case of Theorem 8.1.

\section{Residue formulas for general intersection pairings}

In order to obtain explicit formulas for all the pairings, Witten observes that they can be obtained from those for the $a_{r}$ and $f_{r}$ via his formula [50, (5.20)]. In this section we shall generalize our version of his formula ([50, (4.74)], which is our Theorem 8.1 via the results of Section 2) to give formulas for $\int_{\mathcal{M}(n, d)} \Phi\left(\eta e^{\bar{\omega}}\right)$ where $\eta$ is an equivariant cohomology class that does not simply involve the $\tilde{a}_{r}(X)$ but also involves the $\tilde{b}_{r}^{j}\left(X_{i}^{i}\right)$ and the $\tilde{f}_{r}(X)$ (see Theorems 9.11 and 9.12 below). The key step in the proof is Lemma 9.9, combined with the argument used in Sections 5-8 to prove Theorem 8.1.

In the next section we shall see that Theorem 9.11 yields Witten's formula $[50,(5.20)]$. This will follow from certain equations satisfied by the formula given in Theorem 9.10 (Propositions 10.2 and 10.3).

The next lemma (from [30]) will give an explicit formula for an equivariant cohomology class $\tilde{f}_{r}(X)$ on $M(c)$ such that $\Phi\left(\tilde{f}_{r}(X)\right)=f_{r}$ (cf. Proposition 4.4). In order to state it, we introduce the following notation.

Definition 9.1 (the moment). If $\theta$ is the Maurer-Cartan form on $K$, the moment $J(\theta) \in\left(\Omega^{1}(K) \otimes \mathbf{k}^{*}\right)^{K}$ is defined for $X \in \mathbf{k}$ by

$$
J(\theta)(X)_{k}=-\iota_{X \#} \theta=-\operatorname{Ad}\left(k^{-1}\right) X,
$$

where $X^{\#}$ is the vector field on $K$ given by the left action of $X$ on $K$.

Remark 9.2. See [7, Ch. 7] for an explanation of the role of the moment in the construction of equivariant characteristic classes, via an equivariant version of Chern-Weil theory. Given a principal bundle over a $K$-manifold equipped with a compatible action of $K$ on the total space of the bundle, the moment $J$ plays the same role as the symplectic moment map plays for a principal $U(1)$ bundle $\mathcal{L}$ over a Hamiltonian $K$-manifold with $c_{1}(\mathcal{L})=[\omega]$ (and with a lift of the action of $K$ to the total space of $\mathcal{L}$ ). In particular, the appropriate notion of "equivariant curvature" is the sum of the usual curvature and the moment $J$.

In the next few paragraphs we provide a brief outline of the use of the Bott-Shulman construction (see for instance [10] and other references given in [30]) to obtain equivariant differential forms representing the equivariant characteristic classes $\tilde{f}_{r}(X)$. This material is summarized from [30], which gives a construction of de Rham representatives for equivariant characteristic classes giving rise to the characteristic classes of the universal bundle over $\mathcal{M}(n, d) \times \Sigma$. 
This was accomplished by regarding this bundle (and the classifying space for it) as simplicial manifolds. For more details see [30].

Let $\triangle^{2}=\left\{\left(t_{0}, t_{1}, t_{2}\right) \in[0,1]^{3}: t_{0}+t_{1}+t_{2}=1\right\}$ be the standard 2-simplex. There is a principal $K$-bundle

$$
\triangle^{2} \times K^{3} \stackrel{\pi_{2}}{\longrightarrow} \triangle^{2} \times K^{2}
$$

for which the bundle projection $\pi_{2}: K^{3} \rightarrow K^{2}$ is given by

$$
\pi_{2}\left(g_{0}, g_{1}, g_{2}\right)=\left(g_{0} g_{1}^{-1}, g_{1} g_{2}^{-1}\right) \quad([30,(3.9)]) .
$$

We define a connection $\Theta^{(2)}$ on the total space of this bundle by

$$
\Theta^{(2)}=\sum_{i=0}^{2} t_{i} \theta^{(i)} \in \Omega^{1}\left(\triangle^{2} \times K^{3}\right) \otimes \mathbf{k}
$$

where $\theta^{(i)} \in \Omega^{1}\left(K^{3}\right) \otimes \mathbf{k}$ is the Maurer-Cartan form on the $i^{\text {th }}$ copy of $K$. The curvature

$$
F_{\Theta^{(2)}} \in \Omega^{2}\left(\triangle^{2} \times K^{3}\right) \otimes \mathbf{k}
$$

of the bundle is

$$
F_{\Theta^{(2)}}=\sum_{i} d\left(t_{i} \theta^{(i)}\right)+\left[\Theta^{(2)}, \Theta^{(2)}\right] .
$$

We use this connection and curvature and the Chern-Weil theory of equivariant characteristic classes (see for instance Chapter 7 of [7]) to define an equivariant form on the total space $\triangle^{2} \times K^{3}$ of the bundle, which represents the equivariant characteristic class associated to $\tau_{r}$ in equivariant cohomology. We then integrate this equivariant form over the simplex $\triangle^{2}$. Finally, we may pull this form back to the base space $K^{2}$ via a section $\sigma_{2}: K^{2} \rightarrow K^{3}$ given by

$$
\sigma_{2}\left(k_{1}, k_{2}\right)=\left(k_{1} k_{2}, k_{2}, 1\right) \quad([30,(4.3)]) .
$$

Explicitly, we make the following definition:

Definition 9.3. Let $\Phi_{2}^{K}\left(\tau_{r}\right)=\sigma_{2}^{*} \bar{\Phi}_{2}^{K}\left(\tau_{r}\right) \in \Omega_{K}^{2 r-2}(K \times K)$ (see [30], above (4.3)) where the section $\sigma_{2}$ was as defined above, and

$$
{\overline{\Phi_{2}}}^{K}\left(\tau_{r}\right)=\int_{\triangle^{2}} \tau_{r}\left(F_{\theta(t)}+J(\theta(t))\right) .
$$

Let $\triangle^{1}=\left\{\left(t_{0}, t_{1}\right) \in[0,1]^{2}: t_{0}+t_{1}=1\right\} \cong[0,1]$ be the standard 1-simplex. We shall perform a similar construction using a principal $K$-bundle

$$
\triangle^{1} \times K^{2} \stackrel{\pi_{1}}{\longrightarrow} \triangle^{1} \times K
$$

The bundle projection $\pi_{1}: K^{2} \rightarrow K$ is defined by

$$
\pi_{1}\left(g_{0}, g_{1}\right)=g_{0} g_{1}^{-1} \text {. }
$$


A section $\sigma_{1}: K \rightarrow K^{2}$ of the bundle is given by $\sigma_{1}(k)=(k, 1)$.

On the total space $\triangle^{1} \times K^{2}$ we define a connection

$$
\Theta^{(1)}=\sum_{i=0}^{1} t_{i} \theta^{(i)} \in \Omega^{1}\left(\triangle^{1} \times K^{2}\right) \otimes \mathbf{k}
$$

where $\theta^{(i)} \in \Omega^{1}\left(K^{2}\right) \otimes \mathbf{k}$ is the Maurer-Cartan form on the $i^{\text {th }}$ copy of $K$. The definition of the curvature

$$
F_{\Theta^{(1)}} \in \Omega^{2}\left(\triangle^{1} \times K^{2}\right) \otimes \mathbf{k}
$$

is similar to (9.2). As before, we evaluate the invariant polynomial $\tau_{r}$ on the equivariant curvature and integrate over the simplex $\Delta^{1}$ to get an equivariant form over $K \times K$, and finally we pull this form back to $K$ using the section $\sigma_{1}$ : explicitly, we make the following:

Definition 9.4. We define

$$
\Phi_{1}^{K}\left(\tau_{r}\right)=\sigma_{1}^{*} \bar{\Phi}_{1}^{K}\left(\tau_{r}\right) \in \Omega^{2 r-1}(K),
$$

where

$$
\bar{\Phi}_{1}^{K}\left(\tau_{r}\right)=\int_{\triangle^{1}} \tau_{r}\left(F_{\theta(t)}+J(\theta(t))\right) \in \Omega_{K}^{*}(K \times K) .
$$

Definition 9.5 (equivariant chain homotopy). We define a chain homotopy

$$
I_{K}: \Omega_{K}^{*+1}(\mathbf{k}) \rightarrow \Omega_{K}^{*}(\mathbf{k})
$$

as follows: when $v \in \mathbf{k}$,

$$
\left(I_{K} \beta\right)_{v}=\int_{0}^{1} F_{t}^{*}\left(\iota_{\bar{v}} \beta\right) d t
$$

where $F_{t}: \mathbf{k} \rightarrow \mathbf{k}$ is multiplication by $t$ and $\bar{v}$ is the vector field on $\mathbf{k}$ which takes the constant value $v$.

LEMma 9.6 ([30, Th. 8.1]). The equivariant cohomology class of the equivariant differential form

$$
\tilde{f}_{r}(X)=\operatorname{pr}_{1}^{*} \tilde{f}_{r}(X)_{1}+\operatorname{pr}_{2}^{*} \tilde{f}_{r}(X)_{2}
$$

is a lift of $f_{r} \in H^{2 r-2}(\mathcal{M}(n, d))$ to $H_{K}^{2 r-2}(M(c))$. Here, the maps $\mathrm{pr}_{1}$ and $\mathrm{pr}_{2}$ are the projection maps from $M(c)$ to $K^{2 g}$ and $\mathbf{k}$ defined at (4.4). Also, from $([30,(7.13)])$,

$$
\begin{aligned}
\tilde{f}_{r}(X)_{1}= & \left(\sum_{j=1}^{g}\left(-\mathrm{ev}_{\gamma_{j}^{1}} \times \mathrm{ev}_{x_{j}}+\mathrm{ev}_{\gamma_{j+g}^{0}} \times \mathrm{ev}_{x_{j+g}}\right)^{*} \Phi_{2}^{K}\left(\tau_{r}\right)(X)\right. \\
& +\left(\sum_{j=1}^{g}\left(-\mathrm{ev}_{\gamma_{j+g}^{1}} \times \mathrm{ev}_{x_{j+g}}+\mathrm{ev}_{\gamma_{j}^{0}} \times \mathrm{ev}_{x_{j}}\right)^{*} \Phi_{2}^{K}\left(\tau_{r}\right)(X)\right. \\
\in & \Omega_{K}^{*}\left(K^{2 g}\right)
\end{aligned}
$$


and

$$
\tilde{f}_{r}(X)_{2}=-I_{K}\left(e_{c}^{*} \Phi_{1}^{K}\left(\tau_{r}\right)\right) \in \Omega_{K}^{*}(\mathbf{k})
$$

where $\gamma_{j}^{\alpha}$ (for $\alpha=0,1$ and $j=1, \ldots, 2 g$ ) are certain elements of $\mathbb{F}^{2 g}$ (the free group on $2 g$ generators $x_{1}, \ldots, x_{2 g}$, as in Section 4$)$, whose definition is as given in (7.12) of [30], and for any $z \in \mathbb{F}^{2 g}$, ev $\mathrm{ev}_{z}: K^{2 g} \rightarrow K$ denotes the evaluation map on $z$. Here, $e_{c}: \mathbf{k} \rightarrow K$ is defined by $e_{c}(\Lambda)=c \exp \Lambda$ where the central element $c=e^{2 \pi \mathrm{id} / n} \operatorname{diag}(1, \ldots, 1)$ is as defined in $(2.6)$.

By (9.4),

$$
\begin{aligned}
(9.8) \bar{\Phi}_{1}^{K}\left(\tau_{r}\right)(-X)=\int_{t \in[0,1]} \tau_{r}\left(d t\left(\theta^{(0)}-\theta^{(1)}\right)+t d \theta^{(0)}+(1-t) d \theta^{(1)}\right. \\
+\frac{1}{2}\left[t \theta^{(0)}+(1-t) \theta^{(1)}, t \theta^{(0)}+(1-t) \theta^{(1)}\right] \\
\left.+t \operatorname{Ad}\left(g_{0}^{-1}\right) X+(1-t) \operatorname{Ad}\left(g_{1}^{-1}\right) X\right) .
\end{aligned}
$$

Now

$$
\left.\bar{\Phi}_{1}^{K}\left(\tau_{r}\right)\right|_{T \times T}(-X)=\int_{t \in[0,1]} \tau_{r}\left(d t\left(\theta^{(0)}-\theta^{(1)}\right)+X\right)
$$

since

$$
d \theta^{(i)}+\frac{1}{2}\left[\theta^{(i)}, \theta^{(i)}\right]=0
$$

and the restrictions of $\left[\theta^{(i)}, \theta^{(i)}\right]$ to $T$ vanish. Further

$$
\left.\sigma_{1}^{*} \bar{\Phi}_{1}^{K}\left(\tau_{r}\right)\right|_{T}(-X)=\int_{t \in[0,1]} \tau_{r}(d t \theta+X),
$$

where $\theta$ is the Maurer-Cartan form on $T$. If $\tau_{r}\left(Z_{1}, \ldots, Z_{n-1}\right)=\sum_{I}\left(\tau_{r}\right)_{I} Z^{I}$ where $I=\left(i_{1}, \ldots, i_{n-1}\right)$ is a multi-index and $Z^{I}=Z_{1}^{i_{1}} \ldots Z_{n-1}^{i_{n-1}}$ (in terms of a coordinate system $\left\{Z_{a}=\left\langle\hat{u}_{a}, X\right\rangle, a=1, \ldots, n-1\right\}$ on $\mathbf{t}$, specified by an oriented orthonormal basis $\hat{u}_{a}$ for $\mathbf{t}$ for which $\theta_{a}, a=1, \ldots, n-1$ are the corresponding components of the Maurer-Cartan form $\theta \in \Omega^{1}(T) \otimes \mathbf{t}$ ), then we have

$$
\begin{aligned}
\left.\Phi_{1}^{K}\left(\tau_{r}\right)\right|_{T}(-X) & =\sum_{I} \int_{t \in[0,1]}\left(\tau_{r}\right)_{I}\left(d t \theta_{1}+Z_{1}\right)^{i_{1}} \ldots\left(d t \theta_{n-1}+Z_{n-1}\right)^{i_{n-1}} \\
& =\sum_{a=1}^{n-1} \theta_{a} \partial \tau_{r} / \partial Z_{a}
\end{aligned}
$$

Lemma 9.7. For $\Lambda \in \mathbf{t}$ (in terms of the Maurer-Cartan form $\theta \in$ $\left.\Omega^{1}(T) \otimes \mathbf{t}\right)$

$$
I_{K}\left(e_{c}^{*} \theta\right)_{\Lambda}=\Lambda
$$


Proof. We have

$$
I_{K}\left(e_{c}^{*} \theta\right)_{\Lambda}=\int_{0}^{1} F_{t}^{*}\left(e_{c}^{*} \theta(\bar{\Lambda})\right) d t=\Lambda
$$

since $e_{c}{ }^{*} \theta(\bar{\Lambda}): \mathbf{t} \rightarrow \mathbb{R}$ is the function with constant value $\Lambda$.

Let $q \in S\left(\mathbf{k}^{*}\right)^{K}$ be an invariant polynomial which is given in terms of the elementary symmetric polynomials $\tau_{j}$ by

$$
q(X)=\tau_{2}(X)+\sum_{r=3}^{n} \delta_{r} \tau_{r}(X)
$$

The associated element $\tilde{f}_{(q)}$ of $H_{K}^{*}(M(c))$ is defined by

$$
\tilde{f}_{(q)}=\tilde{f}_{2}+\sum_{r=3}^{n} \delta_{r} \tilde{f}_{r}
$$

Here, the $\delta_{r}$ are formal nilpotent parameters; we $\operatorname{expand} \exp \tilde{f}_{(q)}$ as a formal power series in the $\delta_{r}$. We can alternatively regard the $\delta_{r}$ as real parameters and $\exp \tilde{f}_{(q)}$ as a formal equivariant cohomology class; the integral

$$
\int_{\mathcal{M}(n, d)} \Phi\left(\exp \tilde{f}_{(q)}\right)
$$

and the integral appearing in (10.4) are well defined and are polynomial functions of the $\delta_{j}$, since $\int_{\mathcal{M}(n, d)} \Phi(\eta)=0$ unless $2 \operatorname{deg}(\eta)=\operatorname{dim} \mathcal{M}(n, d)$.

Note that by Lemma 9.6 we can write $\tilde{f}_{(q)}(X)=\operatorname{pr}_{1}^{*} \tilde{f}_{(q)}(X)_{1}+\operatorname{pr}_{2}^{*} \tilde{f}_{(q)}(X)_{2}$ where $\tilde{f}_{(q)}(X)_{1} \in \Omega_{K}^{*}\left(K^{2 g}\right)$ and $\tilde{f}_{(q)}(X)_{2} \in \Omega_{K}^{*}(\mathbf{k})$.

Then we have

LEMMA 9.8. For $X \in \mathbf{t}$, the restriction of $\tilde{f}_{(q)}(-X)_{2}$ to $\mu^{-1}(\mathbf{t})$ is given at $\left(h_{1}, \ldots, h_{2 g}, \Lambda\right) \in \mu^{-1}(\mathbf{t}) \subset K^{2 g} \times \mathbf{k}$ by

$$
\left.\tilde{f}_{(q)}(-X)_{2}\right|_{\mu^{-1}(\mathbf{t})}\left(h_{1}, \ldots, h_{2 g}, \Lambda\right)=-(d q)_{X}(\Lambda) .
$$

Proof.

$$
\begin{aligned}
\left.\tilde{f}_{(q)}(-X)_{2}\right|_{\mu^{-1}(\mathbf{t})} & =-I_{K} e_{c}{ }^{*} \Phi_{1}^{K}(q)(-X) \\
& =-I_{K}\left(\sum_{a=1}^{n-1} \theta^{a} \partial q / \partial Z^{a}\right) \text { by }(9.9) \\
& =-(d q)_{X}(\Lambda) \text { by Lemma } 9.7
\end{aligned}
$$

LEMMA 9.9. Assume that $X \in \mathbf{t}$. Let $\Lambda=\sum_{a=1}^{n-1} m_{a} \hat{e_{a}} \in \Lambda^{I}$ for $m_{a} \in \mathbb{Z}$ (where the simple roots $\hat{e_{a}}$ are as in $\left.(6.2)\right)^{15}$ and let $s_{\Lambda}$ denote the homeomorphism of $M_{\mathbf{t}}(c)$ given by Lemma 4.5. Then on $M_{\mathbf{t}}(c)$

$$
s_{\Lambda}^{*} \tilde{f}_{(q)}(-X)=\tilde{f}_{(q)}(-X)-(d q)_{X}(\Lambda)
$$

\footnotetext{
${ }^{15}$ Note that the $\hat{e_{a}}$ are a basis of $\mathbf{t}$, but not an orthonormal basis.
} 
or equivalently

$$
s_{\Lambda}^{*} \tilde{f}_{(q)}(X)=\tilde{f}_{(q)}(X)+\left(d q_{(o)}\right)_{X}(\Lambda),
$$

where the notation

$$
q_{(o)}(X)=q(-X)
$$

is introduced.

Remark. This result generalizes (4.9).

Proof of Lemma 9.9. Since $\tilde{f}_{(q)}(X)=\operatorname{pr}_{1}^{*} \tilde{f}_{(q)}(X)_{1}+\operatorname{pr}_{2}^{*} \tilde{f}_{(q)}(X)_{2}$, we need to prove the formula for $s_{\Lambda}^{*} \operatorname{pr}_{2}^{*} \tilde{f}_{(q)}(X)_{2}$ where $\tilde{f}_{(q)}(X)_{2}=-I_{K} e_{c}{ }^{*} \Phi_{1}^{K}(q)$ for $\Phi_{1}^{K}(q) \in \Omega_{K}^{*}(K)$. Lemma 9.9 then follows from Lemma 9.8 .

THEOREM 9.10. Suppose $\eta$ is a polynomial in the $\tilde{a}_{r}(X)$ and $\tilde{b}_{r}^{j}(X)$. Let $q \in S\left(\mathbf{k}^{*}\right)^{K}$. Then for any $X \in \mathbf{t}$,

$$
\begin{aligned}
& \int_{N_{n}(V)} \Phi\left(\eta e^{\tilde{f}_{(q)}}\left(e^{\left(d q_{(o)}\right)_{X}\left(\hat{e_{1}}\right)}-1\right) \alpha\right) \\
& =-\sum_{F \in \mathcal{F}:-\left\|\hat{e}_{1}\right\|^{2}<\left\langle\hat{e}_{1}, \mu(F)\right\rangle<0} \operatorname{Res}_{Y_{1}=0} \int_{F} \frac{\Phi_{n-1}\left(\eta e^{\tilde{f}_{(q)}} \alpha\right)}{e_{F}} .
\end{aligned}
$$

Here, we sum over the components $F$ of the fixed point set of $\hat{T}_{1}$ in $P^{-1}(V) \cap \mu^{-1}\left(\hat{\mathbf{t}}_{1}\right) / T_{n-1}$; the notation is as in the statement of Lemma 6.7. The notation $q_{(o)}$ was introduced in the statement of Lemma 9.9. We have defined the map $\Phi_{n-1}$ in Proposition 6.3, and after (7.3).

Proof. This follows from the same proof as for Lemma 6.7, when (4.9) is replaced by its generalization Lemma 9.9 .

We aim to prove the following result by induction:

TheOREm 9.11 (a). For the particular $q$ defined in (9.10),

$$
\begin{aligned}
\int_{N_{n}(c)} \Phi\left(e^{\tilde{f}_{(q)}} \mathcal{D}_{n} \eta\right)= & \frac{(-1)^{n_{+}(g-1)}}{n !} \sum_{w \in W_{n-1}} \operatorname{Res}_{Y_{1}=0} \ldots \operatorname{Res}_{Y_{n-1}=0} \\
& \frac{\int_{\left.T^{2 g} \times\{-[[w \tilde{c}]\}\}\right)}\left(e^{\tilde{f}_{(q)}(X)} \eta(X)\right)}{\mathcal{D}(X)^{2 g-2} \prod_{j=1}^{n-1}\left(\exp -B(-X)_{j}-1\right)},
\end{aligned}
$$

where $\eta$ is a polynomial in the $\tilde{a}_{r}$ and $\tilde{b}_{r}^{j}$ and $B(X)_{j}=-(d q)_{X}\left(\hat{e_{j}}\right)$.

Here we have used the fixed invariant inner product on $\mathbf{k}$ to identify $d q_{X}: \mathbf{t} \rightarrow \mathbb{R}$ with an element of $\mathbf{t}$ and thus define the map $B: \mathbf{t} \rightarrow \mathbf{t}$. The notation $[[\gamma]]$ was introduced in Definition 2.1.

Substitution of $-X$ for $X$ on the right-hand side of the equation in Theorem 9.11 (a) gives the equivalent formulation: 
THEOREM 9.11 (b). In the notation of Theorem 9.11 (a),

$$
\begin{aligned}
\int_{N_{n}(c)} \Phi\left(e^{\tilde{f}_{(q)}} \mathcal{D}_{n} \eta\right)= & \frac{(-1)^{n_{+}(g-1)}}{n !} \sum_{w \in W_{n-1}} \operatorname{Res}_{Y_{1}=0} \ldots \operatorname{Res}_{Y_{n-1}=0} \\
& \frac{\int_{T^{2 g} \times\{-[[w \tilde{c}]\}}\left(e^{\tilde{f}_{(q)}}(-X)\right.}{\mathcal{D}(X)^{2 g-2} \prod_{j=1}^{n-1}\left(1-\exp -B(X)_{j}\right)}
\end{aligned}
$$

Finally we may use Lemma 10.9 and Lemma 10.12 (a) where the restrictions to $T^{2 g}$ of the equivariant cohomology classes $\tilde{f}_{r}(X)$ and $\tilde{b}_{r}^{j}(X)$ are expressed in terms of the basis $\zeta_{a}^{j}$ for $H^{1}\left(T^{2 g}\right.$ ) (for $a=1, \ldots, n-1$ and $j=1, \ldots, 2 g$ ). We also use Lemma 10.10 , where the symplectic volume of $T^{2 g}$ is calculated. These lemmas enable us to compute $\int_{T^{2 g}} e^{\tilde{f}_{(q)}(-X)} \eta(-X)$ and rephrase Theorem 9.11 (b) as follows. (Here we have also reformulated the left-hand side of Theorem 9.11 (b) in terms of the pairings on $\mathcal{M}(n, d)$, using Lemma 6.1.)

THEOREM 9.12. In the notation of Theorem 9.11, (a)

$$
\begin{aligned}
\int_{\mathcal{M}(n, d)} \exp \left(f_{2}+\delta_{3} f_{3}+\ldots+\delta_{n} f_{n}\right) \prod_{r=2}^{n} a_{r}^{m_{r}} \prod_{k_{r}=1}^{2 g}\left(b_{r}^{k_{r}}\right)^{p_{r, k_{r}}} \\
=\frac{(-1)^{n_{+}(g-1)}}{n !} \sum_{w \in W_{n-1}} \operatorname{Res}_{Y_{1}=0} \ldots \operatorname{Res}_{Y_{n-1}=0} \\
\\
\left(\frac{e^{d q_{X}([[w \tilde{c}])}\left(\prod_{r=2}^{n} \tau_{r}(X)^{m_{r}}\right)}{\mathcal{D}(X)^{2 g-2} \prod_{j=1}^{n-1}\left(1-\exp -B(X)_{j}\right)}\right. \\
\quad \times \int_{T^{2 g}} \exp \left\{-\sum_{a, b} \sum_{j=1}^{g} \zeta_{a}^{j} \zeta_{b}^{j+g} \partial^{2} q_{X}\left(\hat{u}_{a}, \hat{u}_{b}\right)\right\} \\
\left.\quad \times \prod_{r=2}^{n} \prod_{k_{r}=1}^{2 g}\left(\sum_{a=1}^{n-1}\left(d \tau_{r}\right)_{X}\left(\hat{u}_{a}\right) \zeta_{a}^{k_{r}}\right)^{p_{r, k_{r}}}\right) .
\end{aligned}
$$

(b) In particular,

$$
\begin{aligned}
\int_{\mathcal{M}(n, d)} & \exp \left(f_{2}+\delta_{3} f_{3}+\ldots+\delta_{n} f_{n}\right) \prod_{r=2}^{n} a_{r}^{m_{r}} \\
= & (-1)^{n_{+}(g-1)} \frac{n^{g}}{n !} \sum_{w \in W_{n-1}} \operatorname{Res}_{Y_{1}=0} \ldots \operatorname{Res}_{Y_{n-1}=0} \\
& \frac{e^{d q_{X}([[w \tilde{c}])} \prod_{r=2}^{n} \tau_{r}(X)^{m_{r}}\left(\operatorname{det} H_{\mathbf{t}}(X)\right)^{g}}{\mathcal{D}(X)^{2 g-2} \prod_{j=1}^{n-1}\left(1-\exp -B(X)_{j}\right)}
\end{aligned}
$$


Remark 9.13. In the preceding theorem, we have used the following notation. The $a_{r}, f_{r}$ and $b_{r}^{j}$ (for $r=2, \ldots, n$ and $j=1, \ldots, 2 g$ ) are generators of the cohomology ring, introduced in Section 2. The $\tau_{r}$ are the elementary symmetric polynomials, and the $\delta_{r}$ are formal nilpotent parameters which were introduced in (9.10). The polynomial $q=\tau_{2}+\sum_{r=3}^{n} \delta_{r} \tau_{r}$ was introduced in (9.10). Its derivative $d q_{X}: \mathbf{t} \rightarrow \mathbb{R}$ is identified with an element of $\mathbf{t}$ via the inner product $\langle\cdot, \cdot\rangle$ on $\mathbf{t}$, and hence $d q: \mathbf{t} \rightarrow \mathbf{t}^{*}$ is identified with a map $B: \mathbf{t} \rightarrow \mathbf{t}$ (see the statement of Theorem 9.11(a)). If $\gamma \in \mathbf{t}$, the notation $[[\gamma]]$ is as introduced in Definition 2.1; it is the unique element in the fundamental domain defined by the simple roots for the translation action on $\mathbf{t}$ of the integer lattice which is equivalent to $\gamma$ under translation by the integer lattice. The $\zeta_{a}^{j}$ are derived from the components of the Maurer-Cartan form $\theta \in \Omega^{1}(T) \otimes \mathbf{t}$ in terms of an orthonormal basis $\left\{\hat{u}_{a}, a=1, \ldots, n-1\right\}$ of $\mathbf{t}$ : they have been identified with a basis of $H^{1}\left(T^{2 g}\right)$. (See Definition 10.6 below.) Finally $\operatorname{det} H_{\mathbf{t}}(X)$ is the determinant of the Hessian of $q: \mathbf{t} \rightarrow \mathbb{R}$, in terms of the coordinates on $\mathbf{t}$ given by the orthonormal basis $\left\{\hat{u}_{a}\right\}$; it is independent of the choice of orthonormal basis.

We note that in Theorem 9.12 the orthonormal basis introduced above could be replaced by a general basis, provided one defines the $\zeta_{a}^{j}$ using that basis, and multiplies the Hessian by a factor due to the change of basis; see Remark 10.1 below.

Remark 9.14. We can replace $f_{2}$ by any nonzero constant scalar multiple $\varepsilon f_{2}$ provided we replace the polynomial $q$ by $q^{\varepsilon}$ where

$$
q^{\varepsilon}(X)=\varepsilon \tau_{2}(X)+\delta_{3} \tau_{3}(X)+\cdots+\delta_{n} \tau_{n}(X)
$$

(cf. Remark 8.3 (b)).

In order to prove Theorem 9.11 and hence Theorem 9.12 we follow the proof of Theorem 8.1 using the following:

LEMMA 9.15. Suppose $\eta$ is a polynomial in the $\tilde{a}_{r}(X)$ and $\tilde{b}_{r}^{j}(X)$. Then for any $X \in \mathbf{t}$ and $q \in S\left(\mathbf{k}^{*}\right)^{K}$ chosen as in (9.10),

$$
\begin{aligned}
& \int_{N_{n}(V)} \Phi\left(\eta \mathcal{D} e^{\tilde{f}_{(q)}} \alpha\right) \\
& =-\sum_{F \in \mathcal{F}:-\left\|\hat{\hat{e}_{1}}\right\|^{2}<\left\langle\hat{e}_{1}, \mu(F)\right\rangle<0} \operatorname{Res}_{Y_{1}=0} \int_{F} \frac{\Phi_{n-1}\left(\mathcal{D} \eta(X) e^{\tilde{f}_{(q)}} \alpha\right)}{e_{F}\left(e^{\left(d q_{(o)}\right)_{X}\left(\hat{e}_{1}\right)}-1\right)},
\end{aligned}
$$

where the notation is as in the statement of Lemma 6.7.

Proof. This follows from Theorem 9.10 by replacement of $\eta$ by

$$
\frac{\eta \mathcal{D}}{\left(e^{\left(d q_{(o)}\right) X\left(\hat{e_{1}}\right)}-1\right)}=\frac{\eta \overline{\mathcal{D}} Y_{1}}{\left(e^{\left(d q_{(o)}\right) X\left(\hat{e_{1}}\right)}-1\right)},
$$


where we have defined $\overline{\mathcal{D}}=\mathcal{D} / Y_{1}$. Notice that $\left(d q_{(o)}\right)_{X}\left(\hat{e_{1}}\right)$ is divisible by $Y_{1}$ : to see this, we observe that if we define the generating functional

$$
P\left(X_{1}, \ldots, X_{n}\right)=\prod_{j=1}^{n}\left(1+t X_{j}\right)=\sum_{r=0}^{n} \tau_{r}\left(X_{1}, \ldots, X_{n}\right) t^{r}
$$

(where the $\tau_{r}$ are the elementary symmetric polynomials) then

$$
d P=\left(\left(1+t X_{1}\right) t d X_{2}+\left(1+t X_{2}\right) t d X_{1}\right) \prod_{j=3}^{n}\left(1+t X_{j}\right)+\mathcal{P}
$$

where $\mathcal{P}$ is a collection of terms involving $d X_{3}, \ldots, d X_{n}$. Evaluating $d P$ on $\hat{e_{1}}=(1,-1,0, \ldots, 0)$ we thus obtain

$$
t^{2}\left(-Y_{1}\right) \prod_{j=3}^{n}\left(1+t X_{j}\right)=\sum_{r=0}^{n} t^{r}\left(d \tau_{r}\right)_{X}\left(\hat{e_{1}}\right) .
$$

It follows that the $\left(d \tau_{r}\right)_{X}\left(\hat{e_{1}}\right)$ (and hence $\left.\left(d q_{(o)}\right)_{X}\left(\hat{e_{1}}\right)\right)$ are divisible by $Y_{1}$. Thus $-\left(d q_{(o)}\right)_{X}\left(\hat{e_{1}}\right)=-Y_{1}(1+\nu)$ where $\nu \in H_{T}^{*}$ has degree at least 1 ; so we have

$$
\left(e^{\left(d q_{(o)}\right)_{X}\left(\hat{e_{1}}\right)}-1\right)=-Y_{1}(1-\tilde{\nu})
$$

where $\tilde{\nu}=\sum_{j \geq 1} \tilde{\nu}_{j}$ is a formal sum of classes $\tilde{\nu}_{j}$ with degree at least 1 in (a completion of) $H_{T}^{*}$. Then the expression

$$
\Phi\left(\frac{\eta \mathcal{D}}{\left(e^{\left(d q_{(o)}\right) X\left(\hat{e_{1}}\right)}-1\right)}\right)
$$

(which appears on the left-hand side of the equation in Theorem 9.10) is well defined. On the right hand side, we may replace

$$
\frac{Y_{1}}{\left(e^{+\left(d q_{(o)}\right) X\left(\hat{e_{1}}\right)}-1\right)}
$$

by $-(1-\tilde{\nu})^{-1}=-\sum_{s \geq 0} \tilde{\nu}^{s}$.

We now use Lemma 9.15 to prove Theorem 9.11 (a) by induction on $n$. The proof follows the outline of the proof of Theorem 8.1 when $q=q_{2}$, with the following modifications:

1. $e^{\bar{\omega}}$ is replaced by $e^{\tilde{f}_{(q)}}$ (and $e^{\omega}$ replaced by $e^{\tilde{f}_{(q)}-\tilde{f}_{2}} e^{\omega}$ ).

2. $\left(d q_{(o)}\right)_{X}\left(\hat{e_{1}}\right)$ replaces $-Y_{1}$, so $e^{\left(d q_{(o)}\right)_{X}\left(\hat{e_{1}}\right)}-1$ replaces $e^{-Y_{1}}-1$. 
3. In particular, $-\left(d q_{(o)}\right)_{X}\left(\hat{e_{1}}\right)=-B(-X)_{1}$ replaces $Y_{1}$ in the identity

$$
\frac{e^{-\delta_{I} Y_{1}}}{1-e^{-Y_{1}}}=\frac{e^{\left(1-\delta_{I}\right) Y_{1}}}{e^{Y_{1}}-1}
$$

which is used in the proof of Proposition 8.4.

We also use the elementary fact that $\left(d q_{(o)}\right)_{X}=-(d q)_{-X}=B(-X)$.

\section{Witten's formulas for general intersection pairings}

In this section we state and prove Witten's formulas (Propositions 10.2 and 10.3 below; cf. [50, §5], in particular the calculations (5.11)-(5.20)), which enabled him to calculate general intersection pairings in terms of those of the form

$$
\int_{\mathcal{M}(n, d)} \prod_{r} a_{r}^{m_{r}} e^{f_{2}}
$$

We shall prove these formulas starting from our explicit formulas for the general intersection pairings (see Theorem 9.11).

Some of the notation in the statements of Propositions 10.2 and 10.3 was introduced at the beginning of Section 9. The invariant polynomial $q$ was defined by (9.10). Using the invariant metric on $\mathbf{k}$, the map $-d q: \mathbf{k} \rightarrow \mathbf{k}^{*}$ may be regarded as a map $B=B^{(2)}+\sum_{r \geq 3} \delta_{r} B^{(r)}: \mathbf{k} \rightarrow \mathbf{k}$, where $B^{(r)}=$ $-d \tau_{r}: \mathbf{k} \rightarrow \mathbf{k}$; we find $B^{(2)}=-d \tau_{2}=\mathrm{id}: \mathbf{k} \rightarrow \mathbf{k}$. (Note that we have put $\tau_{2}(X)=-\frac{1}{2}\langle X, X\rangle$ in terms of the inner product $\langle\cdot, \cdot\rangle$ defined at (2.2).) The other maps $B^{(r)}$ are not linear.

The Hessian of $-q$ is $H$; it is a function from $\mathbf{k}$ to symmetric bilinear forms on $\mathbf{k}$. If $k, l$ run over an orthonormal basis $\left\{\hat{v}_{k}\right\}$ of $\mathbf{k}$ then the Hessian at $X$ is the matrix

$$
H(X)_{k l}=-\left(\partial^{2} q\right)_{X}\left(\hat{v}_{k}, \hat{v}_{l}\right) .
$$

Remark 10.1. In most places in Sections 9 and 10, the orthonormal basis $\left\{\hat{u}_{a}\right\}$ for $\mathbf{t}$ may be replaced by any basis for $\mathbf{t}$ (including the basis $\left\{\hat{e_{a}}, a=1, \ldots, n-1\right\}$, which is of course not orthonormal), and similarly for the orthonormal basis $\left\{\hat{v}_{l}\right\}$ for $\mathbf{k}$. However it is more convenient to define the determinant of the Hessian (given in (10.1)) in terms of an orthonormal basis, since one must otherwise include a normalization factor proportional to the square of the determinant of a matrix whose columns are the basis elements. The second place where it is useful to introduce an orthonormal basis is in the definition of the symplectic form in terms of the generators $\zeta_{a}^{j}$ for the cohomology of $T^{2 g}$; the symplectic form is defined using the inner product $\langle\cdot, \cdot\rangle$ on $\mathbf{t}$, and the formula (Lemma 10.8) for the restriction of the symplectic form to $T^{2 g}$ is cleaner in terms of an orthonormal basis. 
For these reasons we have chosen to use an orthonormal basis for $\mathbf{t}$ throughout Sections 9 and 10, although in many specific instances this basis may be replaced by a general basis. In particular in the statement of our main theorem Theorem 9.12, it is easy to check that the orthonormal basis may be replaced by a general basis, provided that the $\zeta_{a}^{j}$ are also defined using this basis, and that the Hessian is multiplied by the appropriate factor.

We assume the $\delta_{r}$ are formal nilpotent parameters; then the invertibility of $B$ is guaranteed. We write $B^{-1}: \mathbf{k} \rightarrow \mathbf{k}$ as the inverse of $B$. (If the $\delta_{r}$ are nilpotent, the inverse of $B$ may be written as a formal power series in the $\delta_{r}$.)

Proposition 10.2. For any invariant polynomial $\tau \in S\left(\mathbf{k}^{*}\right)^{K}$, the integral

$$
\int_{\mathcal{M}(n, d)} \Phi\left(\tau(-X) \exp \tilde{f}_{(q)}\right)
$$

is equal to the integral

$$
\int_{\mathcal{M}(n, d)} \Phi\left(\tau\left(B^{-1}(-X)\right)\left(\operatorname{det} H\left(B^{-1}(-X)\right)\right)^{g-1}\right) \exp f_{2}
$$

which is of the form that may be calculated by Theorem 8.1.

Proposition 10.3. Let $\tau \in S\left(\mathbf{k}^{*}\right)^{K}$ be an invariant polynomial, so that

$$
\tau=\sum_{m_{2}, \ldots, m_{n}} c_{m_{2}, \ldots, m_{n}} \prod_{r=2}^{n} \tilde{a}_{r}^{m_{r}}
$$

is a polynomial in the $\tilde{a}_{2}, \ldots, \tilde{a}_{n}$. Let $s_{r}^{j}$ be real parameters $($ for $r=2, \ldots, n$ and $j=1, \ldots, 2 g)$. Then,

$$
\begin{aligned}
\int_{\mathcal{M}(n, d)} \Phi\left(\tau(X) \exp \left(\sum_{r=2}^{n} \sum_{j=1}^{2 g} s_{r}^{j} \tilde{b}_{r}^{j}(X)\right) \exp \tilde{f}_{(q)}\right) \\
=\int_{\mathcal{M}(n, d)} \Phi\left(\tau(X) \exp \hat{\tau}(X) \exp \tilde{f}_{(q)}\right) .
\end{aligned}
$$

Here, the invariant polynomial $\hat{\tau}$ on $\mathbf{k}$ is defined (for $X \in \mathbf{t}$ ) by

$$
\hat{\tau}(-X)=-\sum_{a, b=1}^{n-1} \sum_{r, s=2}^{n} \sum_{j=1}^{g} s_{r}^{j} s_{s}^{j+g}\left(d \tau_{r}\right)_{X}\left(\hat{u}_{a}\right)\left(d \tau_{s}\right)_{X}\left(\hat{u}_{b}\right)\left(\partial^{2} q\right)_{a b}^{-1},
$$

where $\left\{\hat{u}_{a}: a=1, \ldots, n-1\right\}$ denotes an oriented orthonormal basis of $\mathbf{t}$; see (10.19) for the definition.

Remark 10.4. Notice that in our conventions on the equivariant cohomology differential and the moment, the construction of [30] described at the 
beginning of Section 9 yields

$$
\tilde{a}_{r}(X)=\tau_{r}(-X) .
$$

Thus $\tau(X)=\sum_{m_{2}, \ldots, m_{n}} c_{m_{2}, \ldots, m_{n}} \prod_{r=2}^{n} \tau_{r}(-X)^{m_{r}}$.

Proposition 10.2 is proved by comparing Theorem 9.12 (b) (applied to (10.2)) with Theorem 8.1 (applied to (10.3)). Proposition 10.3 is obtained by applying Theorem 9.11 (b) to both sides of (10.4) and examining the restrictions to $T^{2 g}$ (which are computed in Lemmas 10.9 and 10.13).

Propositions 10.2 and 10.3 enable us to extract formulas for all pairings, by differentiating the formulas (10.3) and (10.4) with respect to the parameters $\delta_{r}$ and $s_{r}^{j}$ and then setting these parameters equal to zero. In fact, for any nonnegative integers $n_{r}$ (for $r \geq 3$ ) we have

$$
\begin{gathered}
\left(\prod_{r=3}^{n}\left(\frac{\partial}{\partial \delta_{r}}\right)^{n_{r}} \int_{\mathcal{M}(n, d)} \Phi\left(\tau(X) \exp \tilde{f}_{(q)}\right)\right)_{\delta_{3}=\ldots=\delta_{n}=0} \\
\quad=\int_{\mathcal{M}(n, d)} \prod_{r=3}^{n} f_{r}^{n_{r}} \Phi(\tau(X)) \exp f_{2},
\end{gathered}
$$

and likewise for any nonnegative integers $n_{r}$ (with $n_{2}=0$ ) and any choices of $p_{r, j_{r}}=0,1$ we have

$$
\begin{aligned}
& \left(\prod_{r=2}^{n}\left(\frac{\partial}{\partial \delta_{r}}\right)^{n_{r}} \prod_{j_{r}=1}^{2 g}\left(\frac{\partial}{\partial s_{r}^{j_{r}}}\right)^{p_{r, j_{r}}} \int_{\mathcal{M}(n, d)}\right. \\
& \left.\Phi\left(\tau(X) \exp \left(\sum_{r=2}^{n} \sum_{j=1}^{2 g} s_{r}^{j} \tilde{b}_{r}^{j}(X)\right) \exp \tilde{f}_{(q)}\right)\right)_{\delta_{r}=0, s_{r, j}=0} \\
& \quad=\int_{\mathcal{M}(n, d)} \Phi(\tau(X)) \exp f_{2} \prod_{r=2}^{n} f_{r}^{n_{r}} \prod_{j_{r}=1}^{2 g}\left(b_{r}^{j_{r}}\right)^{p_{r, j_{r}}}
\end{aligned}
$$

(where the parameters $\delta_{r}$ and $s_{r}^{j}$ on the left-hand side run over $r=2, \ldots, n$ and $j=1, \ldots, 2 g$ ).

We can use Proposition 10.3 to give an explicit formula for pairings of the form

$$
\int_{\mathcal{M}(n, d)} \Phi\left(\prod_{r=2}^{n} \prod_{k_{r}=1}^{2 g}\left(\tilde{b}_{r}^{k_{r}}(X)\right)^{p_{r, k_{r}}} \tau(X)\right) e^{f_{2}}
$$

where $p_{r, k_{r}}=0$ or 1 . We note that by Proposition 10.3 this equals

$$
\left.\prod_{r=2}^{n} \prod_{k_{r}=1}^{2 g}\left(\frac{\partial}{\partial s_{r}^{k_{r}}}\right)^{p_{r, k_{r}}} \int_{\mathcal{M}(n, d)} \Phi(\tau(X) \exp \hat{\tau}(X)) e^{f_{2}}\right|_{s_{r}^{j}=0 \forall r, j}
$$


where

$$
\hat{\tau}(-X)=-\sum_{a, b=1}^{n-1} \sum_{j=1}^{g} \sum_{r, s=2}^{n} s_{r}^{j} s_{s}^{j+g}\left(d \tau_{r}\right)_{X}\left(\hat{u}_{a}\right)\left(d \tau_{s}\right)_{X}\left(\hat{u}_{b}\right)\left(\partial^{2} q\right)_{a b}^{-1}
$$

Here the $\hat{u}_{a}$ are an oriented orthonormal basis of $\mathbf{t}$. We introduce $T_{r s}: \mathbf{k} \rightarrow \mathbb{R}$ given by $^{16}$

$$
T_{r s}(-X)=-\sum_{a, b=1}^{n-1}\left(d \tau_{r}\right)_{X}\left(\hat{u}_{a}\right)\left(d \tau_{s}\right)_{X}\left(\hat{u}_{b}\right)\left(\partial^{2} q\right)_{a b}^{-1}
$$

Thus we may rewrite (10.7) as

$$
\hat{\tau}(X)=\sum_{r, s=2}^{n} T_{r s}(X)\left(\sum_{j=1}^{g} s_{r}^{j} s_{s}^{j+g}\right) .
$$

We observe that in order for the pairing (10.5) to be nonzero, one requires $p_{r, j}=0$ or 1 for all $r$ and $j$ (since the $b_{r}^{j}$ are of odd degree). Further, in order for the expression (10.6) to yield a nonzero answer, we require for each $j=1, \ldots, g$ that

$$
p_{2, j}+\cdots+p_{n, j}=p_{2, j+g}+\cdots+p_{n, j+g}=l_{j}
$$

for some $l_{j}$. We may then rewrite $(10.6)$ as

$$
\begin{gathered}
\left(\prod_{j=1}^{g}\left(\frac{\partial}{\partial s_{r_{1}}^{j}} \cdots \frac{\partial}{\partial s_{r_{l_{j}}}^{j}}\right)\left(\frac{\partial}{\partial s_{s_{1}}^{j+g}} \cdots \frac{\partial}{\partial s_{s_{l_{j}}}^{j+g}}\right)\right. \\
\left.\int_{\mathcal{M}(n, d)} \Phi(\tau(X) \exp \hat{\tau}(X)) e^{f_{2}}\right)_{s_{r}^{j}=0 \forall r, j}
\end{gathered}
$$

Because $\hat{\tau}$ is quadratic in the $s_{r}^{j}$ and we are setting all the $s_{r}^{j}$ to zero in the end, for each $j$ we may represent the symbols $\frac{\partial}{\partial s_{r}^{j}}$ and $\frac{\partial}{\partial s_{r}^{j+g}}$ as 1 -valent vertices (labelled by $r$ ) in a bipartite graph: there must be exactly one edge coming out of each of these vertices, and these edges must connect the symbol $\frac{\partial}{\partial s_{r}^{j}}$ with a symbol $\frac{\partial}{\partial s_{s}^{j+g}}$ for some $s$. Such bipartite graphs of course correspond to permutations $\sigma_{j}$ of $\left\{1, \ldots, l_{j}\right\}$.

It follows from (10.8) that

$$
\frac{\partial}{\partial s_{r}^{j}} \frac{\partial}{\partial s_{s}^{j+g}} \hat{\tau}(X)=T_{r s}(X) \text { for any } j
$$

${ }^{16}$ Notice that $T_{r s}$ is an invariant polynomial on $\mathbf{k}$. 
so that

$$
\begin{gathered}
\prod_{j=1}^{g}\left(\frac{\partial}{\partial s_{r_{1}}^{j}} \ldots \frac{\partial}{\partial s_{r_{l_{j}}}^{j}}\right)\left(\frac{\partial}{\partial s_{s_{1}}^{j+g}} \cdots \frac{\partial}{\partial s_{s_{l_{j}}}^{j+g}}\right) \int_{\mathcal{M}(n, d)} \Phi(\tau(X) \exp \hat{\tau}(X)) e^{f_{2}} \\
\quad=\int_{\mathcal{M}(n, d)} \Phi\left(\prod_{j=1}^{g} \sum_{\sigma_{j}} T_{r_{1} s_{\sigma_{j}(1)}}(X) \ldots T_{r_{l_{j}} s_{\sigma_{j}\left(l_{j}\right)}}(X) \tau(X)\right) e^{f_{2}}
\end{gathered}
$$

where we sum over all permutations $\sigma_{j}$ of $\left\{1, \ldots, l_{j}\right\}$. Hence we obtain by Remark 8.2 (a) and Lemma 6.1:

TheOREM 10.5.

$$
\begin{aligned}
\int_{\mathcal{M}(n, d)} & \prod_{j=1}^{g} b_{r_{1}}^{j} \ldots b_{r_{l_{j}}}^{j} b_{s_{1}}^{j+g} \ldots b_{s_{l_{j}}}^{j+g} \Phi(\tau(X)) e^{f_{2}} \\
& =\int_{\mathcal{M}(n, d)} \Phi\left(\prod_{j=1}^{g} \sum_{\sigma_{j}} T_{r_{1} s_{\sigma_{j}(1)}}(X) \ldots T_{r_{l_{j}} s_{\sigma_{j}\left(l_{j}\right)}}(X) \tau(X)\right) e^{f_{2}}
\end{aligned}
$$

which equals $(-1)^{n_{+}(g-1)} \frac{n^{g}}{n !}$ times the iterated residue

$$
\begin{aligned}
\sum_{w \in W_{n-1}} & \operatorname{Res}_{Y_{1}}=0 \ldots \operatorname{Res}_{Y_{n-1}}=0 \\
& \frac{\prod_{j=1}^{g} \sum_{\sigma_{j}} T_{r_{1} s_{\sigma_{j}(1)}}(-X) \ldots T_{r_{l_{j}} s_{\sigma_{j}\left(l_{j}\right)}}(-X) \tau(-X) e^{-\langle[[\tilde{c}]], X\rangle}}{\mathcal{D}^{2 g-2}(X)\left(1-\exp \left(-Y_{1}\right)\right) \cdots\left(1-\exp \left(-Y_{n-1}\right)\right)}
\end{aligned}
$$

Let $\hat{u}_{a}(a=1, \ldots, n-1)$ denote an oriented orthonormal basis on $\mathbf{t}$. For $X \in \mathbf{t}$ define coordinates $Z_{a}$ by $Z_{a}=\left(X, \hat{u}_{a}\right)$ so that $X=\sum_{a} Z_{a} \hat{u}_{a}$. Write the Maurer-Cartan form $\theta$ on $T$ as $\theta=\sum_{a} \theta_{a} \hat{u}_{a}$; then the $\theta_{a}$ form a set of generators of $H^{1}(T)$.

Definition 10.6. A set of generators $\left\{\zeta_{a}^{j}\right\}(j=1, \ldots, 2 g ; a=1, \ldots, n-1)$ for $H^{1}\left(T^{2 g}\right)$ is defined by specifying that $\zeta_{a}^{j}=\pi_{j}^{*} \theta_{a}$ where $\pi_{j}: T^{2 g} \rightarrow T$ is the projection onto the $j^{\text {th }}$ copy of $T$.

LEMMA 10.7. $\int_{T} \theta_{1} \wedge \ldots \wedge \theta_{n-1}=\operatorname{vol}(T)$. Here, vol $(T)$ is the Riemannian volume of $T=\mathbf{t} / \Lambda^{I}$ in the metric $\langle\cdot, \cdot\rangle ;$ in other words it is given by $(\operatorname{det} E)^{\frac{1}{2}}$ $=\sqrt{n}$ where $E$ is the $(n-1) \times(n-1)$ matrix (known as the Cartan matrix) given by $E_{a b}=\left\langle\hat{e_{a}}, \hat{e_{b}}\right\rangle$ in terms of the basis for the integer lattice $\Lambda^{I} \subset \mathbf{t}$ over $\mathbb{Z}$ given by the simple roots $\left\{\hat{e_{a}}\right\}, a=1, \ldots, n-1$. 
LEMMA 10.8. The restriction of $\tilde{f}_{(q)}(X)_{1}$ to $T^{2 g}$ is given in terms of the generators $\zeta_{a}^{j}$ of $H^{1}\left(T^{2 g}\right)$ by

$$
\left.\tilde{f}_{(q)}(X)_{1}\right|_{T^{2 g}}=\frac{1}{2} \sum_{a, b} \partial^{2} q\left(\hat{u}_{a}, \hat{u}_{b}\right) \sum_{j=1}^{g}\left(-\zeta_{a}^{j} \zeta_{b}^{j+g}+\zeta_{a}^{j+g} \zeta_{b}^{j}\right),
$$

where the $\left\{\hat{u}_{a}\right\}$ are an oriented orthonormal basis of $\mathbf{t}$.

Proof. We need to understand the restriction of $\tilde{f}_{(q)}$ to $T^{2 g}$. As in (9.6), we have

$$
\begin{aligned}
\tilde{f}_{(q)}(X)_{1}= & \left(\sum_{j=1}^{g}\left(-\mathrm{ev}_{\gamma_{j}^{1}} \times \mathrm{ev}_{x_{j}}+\mathrm{ev}_{\gamma_{j+g}^{0}} \times \mathrm{ev}_{x_{j+g}}\right)^{*} \Phi_{2}^{K}(q)(X)\right. \\
& +\left(\sum_{j=1}^{g}\left(-\mathrm{ev}_{\gamma_{j+g}^{1}} \times \mathrm{ev}_{x_{j+g}}+\mathrm{ev}_{\gamma_{j}^{0}} \times \mathrm{ev}_{x_{j}}\right)^{*} \Phi_{2}^{K}(q)(X) \in \Omega_{K}^{*}\left(K^{2 g}\right)\right.
\end{aligned}
$$

where (after restricting to $T \times T \times T$ )

$$
\left.\bar{\Phi}_{2}^{K}(q)\right|_{T \times T \times T}(-X)=\int_{\left(t_{0}, t_{1}, t_{2}\right) \in \triangle^{2}} q\left(\sum_{k=0}^{2} d t_{k} \theta^{(k)}+X\right) \in H_{T}^{*}(T \times T \times T)
$$

$([30$, above $(5.6)])$ and $\Phi_{2}^{K}(q)=\sigma_{2}^{*} \bar{\Phi}_{2}^{K}(q)$ where $\sigma_{2}:\left(g_{1}, g_{2}\right) \mapsto\left(g_{1} g_{2}, g_{2}, 1\right)$. By (10.12),

$$
\left.\Phi_{2}^{K}(q)\right|_{T \times T}(-X)=-\frac{1}{2} \sum_{a, b} \partial^{2} q\left(\hat{u}_{a}, \hat{u}_{b}\right) \zeta_{a}^{1} \zeta_{b}^{2} \in H_{T}^{*}(T \times T) .
$$

For purposes of evaluation on $T^{2 g}$ the generators $\gamma_{j}^{\tau}$ in (9.6) reduce to

$$
\gamma_{j}^{0}=\gamma_{j+g}^{1}=1, \quad \gamma_{j}^{1}=x_{j+g}, \quad \gamma_{j+g}^{0}=x_{j},
$$

where $x_{1}, \ldots, x_{2 g}$ are the chosen generators of $\mathbb{F}^{2 g}$. So we get from (9.6)

$$
\left.\tilde{f}_{(q)}(X)_{1}\right|_{T^{2 g}}=\sum_{j=1}^{g}\left(-\mathrm{ev}_{x_{j+g}} \times \mathrm{ev}_{x_{j}}+\mathrm{ev}_{x_{j}} \times \mathrm{ev}_{x_{j+g}}\right)^{*} \Phi_{2}^{K}(q)(X) .
$$

We find that

$$
\begin{aligned}
\left.\tilde{f}_{(q)}(-X)_{1}\right|_{T^{2 g}} & =-\frac{1}{2} \sum_{a, b} \partial^{2} q\left(\hat{u}_{a}, \hat{u}_{b}\right) \sum_{j=1}^{g}\left(\zeta_{a}^{j} \zeta_{b}^{j+g}-\zeta_{a}^{j+g} \zeta_{b}^{j}\right) \\
& =-\sum_{a, b} \partial^{2} q\left(\hat{u}_{a}, \hat{u}_{b}\right) \sum_{j=1}^{g} \zeta_{a}^{j} \zeta_{b}^{j+g} .
\end{aligned}
$$

Similarly for $\Lambda \in \mathbf{t} \subset \mathbf{k}$,

$$
\tilde{f}_{(q)}(-X)_{2}(\Lambda)=-(d q)_{X}(\Lambda)
$$

(see Lemma 9.8).

As a result we see immediately that: 
Lemma 10.9. Suppose $c \exp \Lambda=1$. Then

$$
\begin{aligned}
\int_{T^{2 g} \times\{\Lambda\}} \exp \tilde{f}_{(q)}(-X) & =\int_{T^{2 g} \times\{\Lambda\}} \exp \tilde{f}_{q_{2}}(-X)\left(\operatorname{det} H_{\mathbf{t}}(X)\right)^{g} \\
& =e^{-(d q)_{X}(\Lambda)} \int_{T^{2 g}}\left(\operatorname{det} H_{\mathbf{t}}(X)\right)^{g} \exp \omega
\end{aligned}
$$

where $\omega$ is the standard symplectic form on $T^{2 g}$ and the quadratic form $H_{\mathbf{t}}(X)$ is the Hessian of the restriction of $-q$ to $\mathbf{t}$ (evaluated on an oriented orthonormal basis of $\mathbf{t}$ ). In other words,

$$
H_{\mathbf{t}}(X)_{a b}=-\left(\partial^{2} q\right)_{X}\left(\hat{u}_{a}, \hat{u}_{b}\right)
$$

where $\left\{\hat{u}_{a}: a=1, \ldots, n-1\right\}$ is an oriented orthonormal basis for $\mathbf{t}$.

Proof. This follows by integrating

$$
\exp -\sum_{a, b} \partial^{2} q\left(\hat{u}_{a}, \hat{u}_{b}\right) \sum_{j=1}^{g}\left(\zeta_{a}^{j} \zeta_{b}^{j+g}\right)
$$

over $T^{2 g}$. (Notice that $\partial^{2} q\left(\hat{u}_{a}, \hat{u}_{b}\right)$ is symmetric in $a$ and $b$.)

LEMMA 10.10.

$$
\int_{T^{2 g}} \exp \omega=n^{g} .
$$

Proof. This follows from Lemmas 10.7 and 10.8.

In order to prove Proposition 10.2, note that by Theorem 9.11(b),

$$
\int_{N_{n}(c)} \Phi\left(e^{\tilde{f}_{(q)}} \mathcal{D}_{n} \eta\right)
$$

equals $\frac{(-1)^{n}+(g-1)}{n !}$ times the iterated residue

$$
\sum_{w \in W_{n-1}} \operatorname{Res}_{Y_{1}=0} \ldots \operatorname{Res}_{Y_{n-1}}=0 \frac{\int_{T^{2 g} \times\{-[[w \tilde{c}]]\}}\left(e^{\tilde{f}_{(q)}(-X)} \eta(-X)\right)}{\mathcal{D}(X)^{2 g-2}\left(1-e^{-B(X)_{n-1}}\right) \ldots\left(1-e^{-B(X)_{1}}\right)} .
$$

This applies in particular when $\eta(X)=\tau(-X)$ is a linear combination of monomials $\prod_{r} \tilde{a}_{r}^{m_{r}}$ in the $\tilde{a}_{r}$ which does not involve the $\tilde{b}_{r}^{j}$; since $\tilde{a}_{r}(X)=$ $\tau_{r}(-X)$, it is natural to write $\eta(-X)=\tau(X)$. For $\eta$ of this form, the expression above equals

$$
\begin{aligned}
& \frac{(-1)^{n_{+}(g-1)}}{n !} \sum_{w \in W_{n-1}} \operatorname{Res}_{Y_{1}=0} \ldots \operatorname{Res}_{Y_{n-1}=0} \\
& \frac{\int_{T^{2 g} \times\{-[[w \tilde{c}]\}} e^{\tilde{f}_{q_{2}}(-X)}\left(\operatorname{det} H_{\mathbf{t}}(X)\right)^{g} \tau(X)}{\mathcal{D}(X)^{2 g-2}\left(1-e^{-B(X)_{n-1}}\right) \ldots\left(1-e^{-B(X)_{1}}\right)}
\end{aligned}
$$

by Lemma 10.9 . 
We now replace $X$ by $B^{-1}(X)$ (where the transformation $B^{-1}: \mathbf{k} \rightarrow \mathbf{k}$ was defined above Proposition 10.2). This change of variables produces a Jacobian $\left(\operatorname{det} H_{\mathbf{t}}\left(B^{-1}(X)\right)\right)^{-1}$. Thus we obtain

$$
\begin{aligned}
\int_{N_{n}(c)} \Phi\left(\mathcal{D} e^{\tilde{f}_{(q)}} \eta\right)= & \frac{(-1)^{n_{+}(g-1)}}{n !} \sum_{w \in W_{n-1}} \operatorname{Res}_{Y_{1}=0} \ldots \operatorname{Res}_{Y_{n-1}=0} \\
& \left(\left(\operatorname{det} H_{\mathbf{t}}\left(B^{-1}(X)\right)\right)^{g-1}\right. \\
& \left.\times \frac{\int_{T^{2 g} \times\{-[[w \tilde{c}]\}\}} e^{\tilde{f}_{q_{2}}\left(-B^{-1}(X)\right)} \tau\left(B^{-1}(X)\right)}{\mathcal{D}^{2 g-2}\left(B^{-1}(X)\right)\left(1-e^{-Y_{n-1}}\right) \ldots\left(1-e^{-Y_{1}}\right)}\right) .
\end{aligned}
$$

Now we have the statement:

LEMMA 10.11.

$$
\mathcal{D}^{2}\left(B^{-1}(X)\right)=\mathcal{D}^{2}(X)\left(\operatorname{det}\left(-\partial^{2} q\right)_{\mathbf{t}^{\perp}}\right)^{-1},
$$

where $\left(\partial^{2} q\right)_{\mathbf{t}^{\perp}}$ denotes the restriction of the symmetric bilinear form $\left(\partial^{2} q\right)_{X}$ on $\mathbf{k}$ to a symmetric bilinear form on $\mathbf{t}^{\perp}$, which is then identified with a linear map from $\mathbf{t}^{\perp}$ to itself using the fixed invariant inner product.

This lemma will be used in establishing Proposition 10.2 since the Hessian $H$ appearing in that proposition is the Hessian of the ( $K$-invariant) function $-q: \mathbf{k} \rightarrow \mathbb{R}$, which is block diagonal with one block being the Hessian $H_{\mathbf{t}}$ of the restriction of this function to $\mathbf{t}$ and the other block being $-\left(\partial^{2} q\right)_{\mathbf{t}^{\perp}}$.

Proof. We introduce the (orthonormal) basis $X_{\gamma}, Y_{\gamma}$ for $\mathbf{t}^{\perp}$ corresponding to the positive roots $\gamma$, and a corresponding system of coordinates $x_{\gamma}, y_{\gamma}$ on $\mathbf{t}^{\perp}$; we have

$$
\left[X_{\gamma}, X\right]=\gamma(X) Y_{\gamma}, \quad\left[Y_{\gamma}, X\right]=-\gamma(X) X_{\gamma} .
$$

We observe that the map $B$ and its inverse $B^{-1}$ on $\mathbf{k}$ are $K$-equivariant, and map $\mathbf{t}$ to $\mathbf{t}$ and $\mathbf{t}^{\perp}$ to $\mathbf{t}^{\perp}$. Hence

$$
\left(d B^{-1}\right)_{X}\left(\left[X_{\gamma}, X\right]\right)=\left[X_{\gamma}, B^{-1}(X)\right]
$$

and

$$
B^{-1}\left(\operatorname{Ad} \exp \left(X_{\gamma}\right)(X)\right)=\operatorname{Ad} \exp \left(X_{\gamma}\right)\left(B^{-1}(X)\right),
$$

and similarly for $Y_{\gamma}$. We find

$$
\frac{\gamma\left(B^{-1}(X)\right)}{\gamma(X)}=\left(d\left(B^{-1}\right)_{y_{\gamma}}\right)_{X}\left(Y_{\gamma}\right)=\left(d\left(B^{-1}\right)_{x_{\gamma}}\right)_{X}\left(X_{\gamma}\right)
$$


where $\left(B^{-1}\right)_{x_{\gamma}},\left(B^{-1}\right)_{y_{\gamma}}: \mathbf{t}^{\perp} \rightarrow \mathbb{R}$ are the coordinate functions in the directions $x_{\gamma}$ and $y_{\gamma}$. Thus,

$$
\begin{aligned}
\mathcal{D}^{2}\left(B^{-1}(X)\right) & =\mathcal{D}^{2}(X)\left(\operatorname{det} d\left(B^{-1}\right)_{\perp}\right) \\
& =\mathcal{D}^{2}(X)\left(\operatorname{det}-\partial^{2} q\right)_{\mathbf{t}^{\perp}}^{-1}
\end{aligned}
$$

where $d\left(B^{-1}\right)_{\perp}$ is the square matrix of partial derivatives of the $\mathbf{t}^{\perp}$ components of $B^{-1}$ in the directions along $\mathbf{t}^{\perp}$. This completes the proof of Lemma 10.11 .

Proposition 10.2 now follows immediately by using (10.14) and Lemma 10.11 to express (10.2) as an iterated residue, and observing that Theorem 8.1 (in the version given by Remark 8.3 (a)) yields the same iterated residue for (10.3).

Let us now consider the proof of Proposition 10.3. For the rest of this section let $a=1, \ldots, n-1$ index an oriented orthonormal basis $\left\{\hat{u}_{a}\right\}$ of $\mathbf{t}$. We have $\tilde{b}_{r}^{j}(X)=\operatorname{pr}_{1}^{*} \tilde{b}_{r}^{j, 1}$ where $\tilde{b}_{r}^{j, 1}=\operatorname{ev}_{x_{j}}{ }^{*} \Phi_{1}^{K}\left(\tau_{r}\right)$ and $\Phi_{1}^{K}\left(\tau_{r}\right)=\sigma_{1}^{*} \Phi_{1}^{K}\left(\tau_{r}\right)$ where $\Phi_{1}^{K}\left(\tau_{r}\right)$ is as defined by $(9.8)$. Also, $x_{j}$ (for $j=1, \ldots, 2 g$ ) are our chosen set of generators of $H_{1}(\Sigma)$.

Theorem 8.1 applies when $\eta(X)=\tau(X) \exp \sum_{j=1}^{2 g} \sum_{r \geq 2} s_{r}^{j} \tilde{b}_{r}^{j}(X)$ for $s_{r}^{j} \in \mathbb{C}$ and $\tau \in S\left(\mathbf{k}^{*}\right)^{K}$. Define $S^{j} \in S\left(\mathbf{k}^{*}\right)^{K}$ by $S^{j}(X)=\sum_{r \geq 2} s_{r}^{j} \tau_{r}(X)$; we then define $\tilde{b}_{S^{j}}^{j}$ by $\tilde{b}_{S^{j}}^{j}(X)=\sum_{r \geq 2} s_{r}^{j} \tilde{b}_{r}^{j}(X)$.

LEMMA 10.12 (a). The restriction to $T^{2 g}$ of $\tilde{b}_{r}^{j}(-X)$ is $\sum_{a=1}^{n-1}\left(d \tau_{r}\right)_{X}\left(\hat{u}_{a}\right) \zeta_{a}^{j}$ where $\zeta_{a}^{j}$ (for $a=1, \ldots, n-1$ and $j=1, \ldots, 2 g$ ) are the elements of the basis of $H^{1}\left(T^{2 g}\right)$ corresponding to an oriented orthonormal basis $\left\{\hat{u}_{a}\right\}$ for $\mathbf{t}$.

(b) The restriction to $T^{2 g}$ of $\tilde{b}_{S^{j}}^{j}(-X)$ is $\sum_{a=1}^{n-1}\left(d S^{j}\right)_{X}\left(\hat{u}_{a}\right) \zeta_{a}^{j}$.

Proof. We have by (8.21) of [30] that

$$
\tilde{b}_{r}^{j}(X)_{1}=\operatorname{ev}_{x_{j}}{ }^{*} \Phi_{1}^{K}\left(\tau_{r}\right) .
$$

By (9.9),

$$
\left.\Phi_{1}^{K}\left(\tau_{r}\right)\right|_{T^{2 g}}(-X)=\left.\sigma_{1}^{*} \bar{\Phi}_{1}^{K}\left(\tau_{r}\right)\right|_{T^{2 g}}(-X)=\sum_{a=1}^{n-1}\left(d \tau_{r}\right)_{X}\left(\hat{u}_{a}\right) \theta_{a}
$$

so that

$$
\left.\tilde{b}_{r}^{j}(-X)_{1}\right|_{T^{2 g}}=\left.\mathrm{ev}_{x_{j}}{ }^{*} \sigma_{1}^{*} \bar{\Phi}_{1}^{K}\left(\tau_{r}\right)\right|_{T^{2 g}}(-X)=\sum_{a=1}^{n-1}\left(d \tau_{r}\right)_{X}\left(\hat{u}_{a}\right) \zeta_{a}^{j}
$$

since the generators $\zeta_{a}^{j}$ of $H^{1}\left(T^{2 g}\right)$ become identified with the components $\theta_{a}$ of the Maurer-Cartan form on the $j^{\text {th }}$ copy of $T$ in $T^{2 g}$.

LEMMA 10.13. In the notation introduced just before Lemma 10.12,

$$
\int_{T^{2 g}} \exp \tilde{f}_{(q)}(-X) \exp \sum_{j, r} s_{r}^{j} \tilde{b}_{r}^{j}(-X)=\int_{T^{2 g}} \exp \tilde{f}_{(q)}(-X) \exp \hat{\tau}(X)
$$


where

$$
\begin{aligned}
(10.19) \hat{\tau}(X) & =-\sum_{a, b=1}^{n-1} \sum_{j=1}^{g}\left(d S^{j}\right)_{X}\left(\hat{u}_{a}\right)\left(d S^{j+g}\right)_{X}\left(\hat{u}_{b}\right)\left(\partial^{2} q\right)_{a b}^{-1} \\
& =-\sum_{a, b=1}^{n-1} \sum_{r, s=2}^{n} \sum_{j=1}^{g} s_{r}^{j} s_{s}^{j+g}\left(d \tau_{r}\right)_{X}\left(\hat{u}_{a}\right)\left(d \tau_{s}\right)_{X}\left(\hat{u}_{b}\right)\left(\partial^{2} q\right)_{a b}^{-1}
\end{aligned}
$$

Here, $\left\{\hat{u}_{a}: a=1, \ldots, n-1\right\}$ denotes an oriented orthonormal basis of $\mathbf{t}$.

Proof. We need to consider the left-hand side of (10.18), which is

$$
\int_{T^{2 g}} \exp \tilde{f}_{(q)}(-X) \exp \sum_{j, r} s_{r}^{j} \tilde{b}_{r}^{j}(-X) .
$$

By Lemma 10.8 the restriction of $\tilde{f}_{(q)}(-X)$ to $T^{2 g}$ is $\exp -\frac{1}{2} \sum_{a, b} \sum_{j=1}^{g}\left(\partial^{2} q\right)_{X}\left(\hat{u}_{a}, \hat{u}_{b}\right)\left(\zeta_{a}^{j} \zeta_{b}^{j+g}-\zeta_{a}^{j+g} \zeta_{b}^{j}\right)$ while $\exp \sum_{j, r} s_{r}^{j} \tilde{b}_{r}^{j}(-X)$ restricts on $T^{2 g}$ (by Lemma 10.12) to

$$
\exp \sum_{r, j} s_{r}^{j} \sum_{a=1}^{n-1}\left(d \tau_{r}\right)_{X}\left(\hat{u}_{a}\right) \zeta_{a}^{j}
$$

Thus for any given $j=1, \ldots, g$ we must compute the integral

$$
\int_{T^{2}} \exp \left(\sum_{\sigma, \tau} y_{\sigma} A^{\sigma \tau} y_{\tau} / 2+\sum_{\sigma} y_{\sigma} B_{j}^{\sigma}\right)
$$

where $\sigma$ runs over pairs $(a, i)$ for $a=1, \ldots, n-1$ and $i=0,1$ (where $i=0$ corresponds to $j$ and $i=1$ to $j+g$ ) and $y_{a, i}=\zeta_{a}^{j+g i}$. Here, the matrix $A$ is given by

$$
A^{a 0, b 1}=-\left(\partial^{2} q\right)_{X}\left(\hat{u}_{a}, \hat{u}_{b}\right)=-A^{a 1, b 0} ; A^{a 0, b 0}=A^{a 1, b 1}=0 ;
$$

thus the Pfaffian of $A$ (whose square is $\operatorname{det} A$ ) is given by

$$
\operatorname{Pf}(A)=\operatorname{det}\left(-\left.\partial^{2} q\right|_{\mathbf{t}}\right)
$$

For $j=1, \ldots, g$ the vector $B_{j}$ is

$$
B_{j}^{a 0}=-\left(\partial S^{j}\right)_{X}\left(\hat{u}_{a}\right) ; \quad B_{j}^{a 1}=-\left(\partial S^{j+g}\right)_{X}\left(\hat{u}_{a}\right) .
$$

The result is that

$$
\begin{aligned}
\int_{T^{2}} \exp & \left(\sum_{\sigma, \tau} y_{\sigma} A^{\sigma \tau} y_{\tau} / 2+\sum_{\sigma} y_{\sigma} B_{j}^{\sigma}\right) \\
= & \int_{T^{2}} \exp \left(\sum_{\sigma, \tau} y_{\sigma} A^{\sigma \tau} y_{\tau} / 2\right) \exp \left(-B_{j}^{t} A^{-1} B_{j}\right) / 2 \\
= & \operatorname{Pf}(A) \exp \left(-B_{j}^{t} A^{-1} B_{j}\right) / 2
\end{aligned}
$$


where $B_{j}^{t}$ denotes the transpose of the vector $B_{j}$. Thus we find that (10.20) becomes

$$
\operatorname{det}\left(-\left.\partial^{2} q\right|_{\mathbf{t}}\right)^{g} \exp \left(\sum_{a, b=1}^{n-1} \sum_{j=1}^{g}\left(d S^{j}\right)_{X}\left(\hat{u}_{a}\right)\left(d S^{j+g}\right)_{X}\left(\hat{u}_{b}\right)\left(\partial^{2} q\right)_{a, b}^{-1}\right),
$$

which equals the right-hand side of (10.18). This completes the proof of Lemma 10.13.

Proposition 10.3 follows from Theorem 9.11 once we have shown that

$$
\int_{T^{2 g}} \exp \tilde{f}_{(q)}(-X) \exp \sum_{r \geq 2} s_{r}^{j} \tilde{b}_{r}^{j}(-X)=\int_{T^{2 g}} \exp \tilde{f}_{(q)}(-X) \exp \hat{\tau}(X)
$$

where $\hat{\tau}$ is given by (10.7). This is now clear from Lemma 10.13 .

\section{The Verlinde formula}

The Verlinde formula is a formula for the dimension $D_{n, d}(g, k)$ of the space of holomorphic sections of powers of $\mathcal{L}$, where $\mathcal{L}$ is a particular line bundle over $\mathcal{M}(n, d)$; it has been proved by Beauville and Laszlo [6], Faltings [20], Kumar, Narasimhan and Ramanathan [38] and Tsuchiya, Ueno and Yamada [48]. In this section we show how the Verlinde formula follows from our formula (Theorem 8.1) for intersection pairings in $\mathcal{M}(n, d)$.

A line bundle $\mathcal{L}$ over $\mathcal{M}(n, d)$ may be defined for which $c_{1}(\mathcal{L})=n f_{2}$, since $n f_{2} \in H^{2}(\mathcal{M}(n, d), \mathbb{Z})$ (see [16]). As described in Section 1, this bundle is the determinant line bundle. Whenever $k$ is a positive integer divisible by $n$, we then define

$$
D_{n, d}(g, k)=\operatorname{dim} H^{0}\left(\mathcal{M}(n, d), \mathcal{L}^{k / n}\right) .
$$

Let us introduce

$$
r=k+n
$$

let us also introduce the highest root $\gamma_{\max }$, which is given by $\gamma_{\max }(X)=$ $X_{n}-X_{1}$ or $\gamma_{\max }=e_{1}+e_{2}+\cdots+e_{n-1}$. We then make the following definition:

Definition 11.1. The Verlinde function $V_{n, d}(g, k)$ is given by

$$
V_{n, d}(g, k)=\sum_{\lambda \in \Lambda_{\mathrm{reg}}^{w} \cap \mathbf{t}_{+}:\left\langle\lambda, \gamma_{\max }\right\rangle<r} \frac{e^{-2 \pi i\langle\lambda-\rho, \tilde{c}\rangle}}{\left(S_{0 \lambda}(k)\right)^{2 g-2}}
$$

where $\rho$ is half the sum of the positive roots and

$$
S_{0 \lambda}(k)=\frac{1}{\sqrt{n} r^{(n-1) / 2}} \prod_{\gamma>0} 2 \sin \pi\langle\gamma, \lambda\rangle / r .
$$


(See $[21,(\mathrm{~A} .44)]$ and $[49,(3.16)]$.$) Verlinde's conjecture says that the$ Verlinde function specifies the dimension of the space of holomorphic sections of $\mathcal{L}^{k / n}$ :

THEOREM 11.2 (Verlinde's conjecture).

$$
D_{n, d}(g, k)=V_{n, d}(g, k) \text {. }
$$

We shall show how to extract Verlinde's conjecture from our previous results: an outline of the method we use was given by Szenes ([45, (§4.2)]).

In fact $H^{i}\left(\mathcal{M}(n, d), \mathcal{L}^{m}\right)=0$ for all $i>0$ and $m>0$ by an argument using the Kodaira vanishing theorem and the facts that $\mathcal{L}$ is a positive line bundle and the canonical bundle of $\mathcal{M}(n, d)$ is equal to $\mathcal{L}^{-2}$ (see $[5, \S 5]$ and Théorème $\mathrm{F}$ of [16]); now $D_{n, d}(g, k)$ is given for $k>0$ by the Riemann-Roch formula:

$$
D_{n, d}(g, k)=\int_{\mathcal{M}(n, d)} \operatorname{ch} \mathcal{L}^{k / n} \operatorname{td} \mathcal{M}(n, d) .
$$

We use the following results to convert (11.2) into a form to which we may apply our previous results.

Lemma 11.3. For any complex manifold $M$ the Todd class of $M$ is given by

$$
\operatorname{td}(M)=e^{c_{1}(M) / 2} \hat{A}(M)
$$

where $c_{1}(M)$ is the first Chern class of the holomorphic tangent bundle of $M$, and $\hat{A}(M)$ is the A-roof genus of $M$.

Proof. See for example [22, pages 97-99].

Proposition 11.4.

$$
\hat{A}(\mathcal{M}(n, d))=\Phi\left(\prod_{\gamma>0} \frac{\gamma(X) / 2}{\sinh \gamma(X) / 2}\right)^{2 g-2}
$$

Proof. This is proved by Newstead ${ }^{17}$ in [42].

LEMMA 11.5. We have

$$
c_{1}(\mathcal{M}(n, d))=2 n f_{2}
$$

\footnotetext{
${ }^{17}$ Newstead writes the details of the proof only for $n=2$ but the same proof yields the result for general $n$.
} 
Proof. This is proved in $[16$, Th. F].

Of course the Chern character of $\mathcal{L}^{k / n}$ is given by $\operatorname{ch} \mathcal{L}^{k / n}=e^{k f_{2}}$. Thus we obtain:

Corollary 11.6. The quantity $D_{n, d}(g, k)$ is given by

$$
D_{n, d}(g, k)=\int_{\mathcal{M}(n, d)} e^{(k+n) f_{2}} \Phi\left(\prod_{\gamma>0} \frac{\gamma(X)}{e^{\gamma(X) / 2}-e^{-\gamma(X) / 2}}\right)^{2 g-2} .
$$

Proof. This follows immediately from (11.2), Lemmas 11.3 and 11.5 and Proposition 11.4.

TheOREM 11.7. We have

$$
\begin{aligned}
D_{n, d}(g, k)= & \frac{(-1)^{n_{+}(g-1)}}{n !} \sum_{w \in W_{n-1}} \operatorname{Res}_{Y_{1}=0} \ldots \operatorname{Res}_{Y_{n-1}=0} \\
& \left(e^{r\langle[[w \tilde{c}], X\rangle} \int_{T^{2 g}} e^{r \omega} \prod_{\gamma>0}\left(\frac{\gamma(X)}{e^{\gamma(X) / 2}-e^{-\gamma(X) / 2}}\right)^{2 g-2}\right. \\
& \left.\times \frac{1}{\prod_{j=1}^{l}\left(e^{r Y_{j}}-1\right) \mathcal{D}(X)^{2 g-2}}\right) .
\end{aligned}
$$

Proof. This comes straight from Corollary 11.6 and Theorem 8.1. Note that because the factor $e^{f_{2}}$ in the statement of Theorem 8.1 has been replaced by $e^{r f_{2}}$, it is necessary to replace $e^{\langle[[w \tilde{c}], X\rangle}$ by $e^{r\langle[[w \tilde{c}]], X\rangle}$, and $e^{Y_{j}}-1$ by $e^{r Y_{j}}-1$ (cf. Remark 8.3 (c)).

We introduce $Z_{j}=\exp Y_{j}$. Since for any $w \in W_{n-1}$,

$$
[[w \tilde{c}]]=[[w \tilde{c}]]_{1} \hat{e_{1}}+[[w \tilde{c}]]_{2} \hat{e_{2}}+\ldots+[[w \tilde{c}]]_{n-1} e_{n-1}
$$

(as in the statement of Proposition 2.2) with $n[[w \tilde{c}]]_{j} \in \mathbb{Z}$ for all $j$, and $0 \leq$ $[[w \tilde{c}]]_{j}<1$ for all $j$, we obtain

$$
e^{r\langle[[w \tilde{c}]], X\rangle}=Z_{1}^{[[w \tilde{c}]]_{1} r} Z_{2}^{[[w \tilde{c}]]_{2} r} \ldots Z_{n-1}^{[[w \tilde{c}]]_{n-1} r} .
$$

(Recall that $k$ and $r$ are divisible by $n$ so $e^{r\langle\tilde{c}, X\rangle}$ is a well-defined single-valued function of $Z_{1}, \ldots, Z_{n-1}$.) Thus we can equate $D_{n, d}(g, k)$ with

$$
\begin{gathered}
(11.4) \frac{(-1)^{n_{+}(g-1)}}{n !} \sum_{w \in W_{n-1}} \operatorname{Res}_{Z_{1}=1} \ldots \operatorname{Res}_{Z_{n-1}=1}\left(\left(\prod_{j=1}^{n-1} \frac{1}{Z_{j}}\right) \int_{T^{2 g}} e^{r \omega}\right. \\
\left.\times \frac{Z_{1}^{[[w \tilde{c}]]_{1} r} Z_{2}^{[[w \tilde{c}]]_{2} r} \ldots Z_{n-1}^{[[w \tilde{c}]]_{n-1} r}}{\prod_{\gamma>0}\left(\tilde{\gamma}^{1 / 2}-\tilde{\gamma}^{-1 / 2}\right)^{2 g-2}\left(Z_{1}^{r}-1\right) \ldots\left(Z_{n-1}^{r}-1\right)}\right)
\end{gathered}
$$




$$
\begin{aligned}
= & \frac{(-1)^{n-1+n_{+}(g-1)}}{n !} \sum_{w \in W_{n-1}} \operatorname{Res}_{Z_{1}=1} \ldots \operatorname{Res}_{Z_{n-1}=1}\left(\left(\prod_{j=1}^{n-1} \frac{1}{Z_{j}}\right)\right. \\
& \left.\times \int_{T^{2 g}} e^{r \omega} \frac{Z_{1}^{-[[w \tilde{c}]]_{1} r} Z_{2}^{-\left[[w \tilde{c}]_{2} r\right.} \ldots Z_{n-1}^{-[[w \tilde{c}]]_{n-1} r}}{\prod_{\gamma>0}\left(\tilde{\gamma}^{1 / 2}-\tilde{\gamma}^{-1 / 2}\right)^{2 g-2}\left(Z_{1}^{-r}-1\right) \ldots\left(Z_{n-1}^{-r}-1\right)}\right) .
\end{aligned}
$$

Here, we have introduced $\tilde{\gamma}$ defined (for the root $\gamma=e_{r}+e_{r+1}+\cdots+e_{s-1}$ ) by

$$
\tilde{\gamma}\left(Z_{1}, \ldots, Z_{n-1}\right)=Z_{r} \ldots Z_{s-1} .
$$

We also have:

LEMMA 11.8.

$$
\int_{T^{2}} e^{\omega}=n
$$

and hence

$$
\int_{T^{2 g}} e^{r \omega}=r^{(n-1) g} n^{g} .
$$

Proof. This follows from Lemma 10.10.

The following may be proved by the same method as in Section 2 (see [45]):

Proposition 11.9. Suppose $f$ is the meromorphic function on the complexification $T^{\mathbb{C}}$ of $T$ defined by

$$
f(Z)=(-1)^{n-1}(-1)^{n_{+}(g-1)} r^{(n-1)(g-1)} n^{g-1} \frac{Z_{1}^{-\tilde{c}_{1} r} \ldots Z_{n-1}^{-\tilde{c}_{n-1} r}}{\prod_{\gamma>0}\left(\tilde{\gamma}^{1 / 2}-\tilde{\gamma}^{-1 / 2}\right)^{2 g-2}}
$$

Then,

$$
\begin{gathered}
\frac{1}{(n-1) !} \operatorname{Res}_{Z_{1}=1} \ldots \operatorname{Res}_{Z_{n-1}=1} \sum_{w \in W_{n-1}} \prod_{j=1}^{n-1}\left(\frac{r}{Z_{j}}\right) \frac{[[w f]](Z)}{\prod_{j=1}^{n-1}\left(Z_{j}^{-r}-1\right)} \\
=\sum_{\lambda \in \Lambda_{\mathrm{reg}}^{w} \cap \mathbf{t}_{+}:\left\langle\lambda, \gamma_{\max }\right\rangle<r} f(\exp 2 \pi i \lambda / r) .
\end{gathered}
$$

Here, $W_{n-1}$ is the permutation group on $\{1, \ldots, n-1\}$ which is (isomorphic to) the Weyl group of $\mathrm{SU}(n-1)$, and $[[w f]]$ is the function

$$
[[w f]](Z)=(-1)^{n-1}(-1)^{n_{+}(g-1)} r^{(n-1)(g-1)} n^{g-1} \frac{Z_{1}^{-[[w \tilde{c}]]_{1} r} \ldots Z_{n-1}^{-[[w \tilde{c}]]_{n-1} r}}{\prod_{\gamma>0}\left(\tilde{\gamma}^{1 / 2}-\tilde{\gamma}^{-1 / 2}\right)^{2 g-2}} .
$$

Remark. Notice that

$$
\sum_{\lambda \in \Lambda_{\mathrm{reg}}^{w} \cap \mathbf{t}_{+}:\left\langle\lambda \lambda, \gamma_{\max }\right\rangle<r} f(\exp 2 \pi i \lambda / r)=\frac{1}{n-1} \cdot \sum_{m_{j}=1}^{r-1} f\left(e^{2 \pi i\left(\sum_{j} m_{j} w_{j}\right) / r}\right) .
$$


(Here, the $w_{j}$ are the fundamental weights, which are dual to the simple roots.) The set $\left\{X \in \mathbf{t}: X=\sum_{j} \lambda_{j} \hat{e}_{j}, 0 \leq \lambda_{j}<1, j=1, \ldots, n-1\right\}$ is a fundamental domain for the action of the integer lattice $\Lambda^{I}$ on $\mathbf{t}$, while the set $\left\{X \in \mathbf{t}_{+} \subset \mathbf{t}: \gamma_{\max }(X)<1\right\}$ is a fundamental domain for the affine Weyl group $W_{\text {aff }}$ (the semidirect product of the Weyl group and the integer lattice), and $\Lambda^{I}$ has index $(n-1)$ ! (rather than $n$ !) in $W_{\text {aff (in other words a fundamental }}$ domain for $\Lambda^{I}$ contains $(n-1)$ ! fundamental domains for $\left.W_{\text {aff }}\right)$. This difference accounts for the factor $1 /(n-1)$ ! in Proposition 11.9 which replaces the factor $1 / n$ ! in its analogue Proposition 2.2 .

Applying Proposition 11.9 we find (recalling from Section 2 that $(-1)^{n-1}=$ $c^{\rho}$ when $n$ and $d$ are coprime) that

$$
\begin{aligned}
D_{n, d}(g, k)= & (-1)^{n_{+}(g-1)} r^{(n-1)(g-1)} n^{g-1} c^{\rho} \sum_{\lambda \in \Lambda_{\mathrm{reg}}^{w} \cap \mathbf{t}_{+}:\left\langle\lambda, \gamma_{\max }\right\rangle<r} \\
& \times \frac{e^{-2 \pi i\langle\tilde{c}, \lambda\rangle}}{\prod_{\gamma>0}\left(e^{2 \pi i\left(\frac{\gamma}{2 r}, \lambda\right\rangle}-e^{-2 \pi i\left\langle\frac{\gamma}{2 r}, \lambda\right\rangle}\right)^{2 g-2}} .
\end{aligned}
$$

This gives

$$
\begin{aligned}
D_{n, d}(g, k)= & (-1)^{n_{+}(g-1)} r^{(n-1)(g-1)} n^{g-1} \sum_{\lambda \in \Lambda_{\mathrm{reg}}^{w} \cap \mathbf{t}_{+}:\left\langle\lambda, \gamma_{\max }\right\rangle<r} \\
& \times \frac{e^{-2 \pi i\langle\tilde{c}, \lambda-\rho\rangle}}{\prod_{\gamma>0}(2 i \sin \pi\langle\gamma, \lambda\rangle / r)^{2 g-2}} \\
= & r^{(n-1)(g-1) n^{g-1} \sum_{\lambda \in \Lambda_{\mathrm{reg}}^{w} \cap \mathbf{t}_{+}:\left\langle\lambda, \gamma_{\max }\right\rangle<r}} \\
& \times \frac{e^{-2 \pi i\langle\tilde{c}, \lambda-\rho\rangle}}{\prod_{\gamma>0}\left(2 \sin \frac{\pi\langle\gamma, \lambda\rangle}{r}\right)^{2 g-2}} .
\end{aligned}
$$

Comparing this with Definition 11.1, we see that $D_{n, d}(g, k)=V_{n, d}(g, k)$. This completes the proof of Theorem 11.2.

Princeton University, Princeton, NJ

Current address: McGill University, Montreal, Quebec, Canada

E-mail address: JEFFREY@MATH.MCGILL.CA

BALliol COLLEge, OXFord OX1 3BJ, UK

E-mail address: FRANCES.KIRWAN@BALLIOL.OXFORD.AC.UK

\section{REFERENCES}

[1] M. F. Aтryah, Convexity and commuting Hamiltonians, Bull. London Math. Soc. 14 (1982), 1-15.

[2] M. F. Atryah and R. Botr, The Yang-Mills equations over Riemann surfaces, Phil. Trans. Roy. Soc. Lond. A308 (1983), 523-615. 
[3] The moment map and equivariant cohomology, Topology 23 (1984), 1-28.

[4] S. Axelrod, S. Della Pietra, and E. Witten, Geometric quantization of ChernSimons gauge theory, J. Diff. Geom. 33 (1991), 787-902.

[5] A. BEAUVILLE, Vector bundles on curves and generalized theta functions: recent results and open problems, preprint alg-geom/9404001 (1994) Math. Sci. Res. Inst. Publ. 28, Cambridge Univ. Press, 1995.

[6] A. Beauville and Y. Laszlo, Conformal blocks and generalized theta functions, Commun. Math. Phys. 164 (1994), 385-519.

[7] N. Berline, E. Getzler, and M. Vergne, Heat Kernels and Dirac Operators, SpringerVerlag (Grundlehren vol. 298), 1992.

[8] N. Berline et M. Vergne, Classes caractéristiques équivariantes. Formules de localisation en cohomologie équivariante, C. R. Acad. Sci. Paris Sér. I Math. 295 (1982), $539-541$.

[9] Z Zéros d'un champ de vecteurs et classes caractéristiques équivariantes, Duke Math. J. 50 (1983), 539-549.

[10] R. Botr, H. Shulman, and J. StashefF, On the de Rham theory of certain classifying spaces, Adv. Math. 20 (1976), 43-56.

[11] R. Вотт and L. Tu, Differential Forms in Algebraic Topology (Graduate Texts in Mathematics 82), Springer-Verlag, 1982.

[12] T. Bröcker and T. том Dieck, Representations of Compact Lie Groups (Graduate Texts in Mathematics vol. 98) Springer-Verlag, 1985.

[13] U. V. Desale and S. Ramanan, Poincaré polynomials of the variety of stable bundles, Math. Ann. 216 (1975), 233-244.

[14] A. Dold, Lectures on Algebraic Topology, Springer-Verlag (Grundlehren vol. 200), 1980 .

[15] S. K. Donaldson, Gluing techniques in the cohomology of moduli spaces, in Topological Methods in Modern Mathematics (Proc. 1991 conf., Stony Brook, NY in honour of the sixtieth birthday of J. Milnor), Publish or Perish, 1993, 137-170.

[16] J.-M. Drezet et M. S. Narasimhan, Groupe de Picard des variétés de modules de fibrés semi-stables sur les courbes algébriques, Invent. Math. 97 (1989), 53-94.

[17] J. J Duistermaat and G. Heckman, On the variation in the cohomology of the symplectic form of the reduced phase space, Invent. Math. 69 (1982), 259-268; Addendum, 72 (1983), 153-158.

[18] J. J. Duistermant, Equivariant cohomology and stationary phase, Utrecht preprint no. 817 (1993); in Symplectic Geometry and Quantization (Sanda and Yokohama, 1993), Y. Maeda, H. Omori and A. Weinstein, eds.; Contemp. Math., Amer. Math. Soc. 179 (1994), 45-62.

[19] M. Duflo et M. Vergne, Orbites coadjointes et cohomologie équivariante, in M. Duflo, N. V. Pedersen, M. Vergne (eds.), The Orbit Method in Representation Theory (Progress in Mathematics, vol. 82), Birkhäuser, (1990) 11-60.

[20] G. Faltings, A proof for the Verlinde formula, J. Alg. Geom. 3 (1994), 347-374.

[21] D. Gepner and E. Witten, String theory on group manifolds, Nucl. Phys. B 278 (1986), 493-549.

[22] P. B. GILKey, Invariance Theory, the Heat Equation and the Atiyah-Singer Index Theorem, Publish or Perish, 1984.

[23] V. Guillemin and J. Kalkman, The Jeffrey-Kirwan localization theorem and residue operations in equivariant cohomology, J. Reine Angew. Math. 470 (1996), 123-142.

[24] V. Guillemin and S. SternberG, Convexity properties of the moment mapping, Invent. Math. 67 (1982), 491-513.

[25] G. Harder and M. S. Narasimhan, On the cohomology groups of moduli spaces of vector bundles over curves, Math. Ann. 212 (1975), 215-248. 
[26] S. Helgason, Differential Geometry, Lie Groups and Symmetric Spaces, Academic Press, 1978.

[27] L. Hörmander, The Analysis of Linear Partial Differential Operators I (Grundlehren v. 256), Springer, 1983.

[28] L. C. JeFFrey, Extended moduli spaces of flat connections on Riemann surfaces, Math. Annalen 298 (1994), 667-692.

[29] - Symplectic forms on moduli spaces of flat connections on 2-manifolds, in Proc. Georgia Intern. Topology Conf. (Athens, GA, 1993), ed. W. Kazez. AMS (1997) 268-281.

[30] Group cohomology construction of the cohomology of moduli spaces of flat connections on 2-manifolds, Duke Math. J. 77 (1995), 407-429.

[31] L. C. JEFFREY and F. C. KirWAN, Localization for nonabelian group actions, Topology 34 (1995), 291-327.

[32] Intersection pairings in moduli spaces of holomorphic bundles on a Riemann surface, Elec. Res. Announ. AMS 1 (1995), 57-71.

[33] L Localization and the quantization conjecture, Topology 36 (1997), 647-693.

[34] J. KaLKMAN, BRST model for equivariant cohomology and representatives for the equivariant Thom class, Commun. Math. Phys. 153 (1993), 447-463.

[35] C Cohomology rings of symplectic quotients, J. Reine Angew. Math. 458 (1995), 37-52.

[36] F. KIRwan, Cohomology of Quotients in Symplectic and Algebraic Geometry, Math. Notes 31 Princeton University Press (1984).

[37] The cohomology rings of moduli spaces of bundles over Riemann surfaces, J. A. M. S. 5 (1992), 853-906.

[38] S. Kumar, M. S. Narasimhan, and A. Ramanathan, Infinite Grassmannians and moduli spaces of $G$-bundles, Math. Ann. 300 (1994), 41-75.

[39] S. K. MarTin, Topological localization, preprint (1994).

[40] V. Mathai and D. Quillen, Superconnections, Thom classes, and equivariant differential forms, Topology 25 (1986), 85-110.

[41] M. S. NARASimhan and C. S. SEshadri, Stable and unitary vector bundles on a compact Riemann surface, Ann. of Math. 82 (1965), 540-567.

[42] P. Newstead, Characteristic classes of stable bundles of rank 2 over an algebraic curve, Trans. Amer. Math. Soc. 169 (1972), 337-345.

[43] D. G. Quillen, Determinants of Cauchy-Riemann operators over a Riemann surface, Funct. Anal. Appl. 19 (1985), 31-34.

[44] J. Roe, Elliptic Operators, Topology and Asymptotic Methods, Longman (Pitman Research Notes vol. 179), 1988.

[45] A. SzEnes, The combinatorics of the Verlinde formulas, alg-geom/9402003 in Vector Bundles in Algebraic Geometry, ed. N. Hitchin (LMS Lecture Note Series 208), 1995; A. Szenes, private communication.

[46] - Iterated residues and multiple Bernoulli polynomials, Mittag-Leffler Institute preprint (1997), hep-th/9707114.

[47] M. Thaddeus, Conformal field theory and the cohomology of the moduli space of stable bundles, J. Diff. Geom. 35 (1992), 131-149.

[48] A. TsuchiYa, K. Ueno, and Y. Yamada, Conformal field theory on universal family of stable curves with gauge symmetries, Advanced Studies in Pure Math. 19, Academic Press (1989), 459-566.

[49] E. Witten, On quantum gauge theories in two dimensions, Comm. Math. Phys. 141 (1991), 153-209. 
[50] Two dimensional gauge theories revisited, preprint hep-th/9204083; J. Geom. Phys. 9 (1992), 303-368.

[51] S. Wu, An integration formula for the square of moment maps of circle actions, preprint hep-th/9212071: Lett. Math. Phys. 29 (1993), 311-328.

(Received August 5, 1996)

(Revised November 30, 1997) 